\title{
Influence of micro-environments on pre-school children's energy balance-related behaviours and weight status
}

Citation for published version (APA):

Gubbels, J. S. (2010). Influence of micro-environments on pre-school children's energy balance-related behaviours and weight status. [Doctoral Thesis, Maastricht University]. Maastricht. https://doi.org/10.26481/dis.20101119jg

Document status and date:

Published: 01/01/2010

DOI:

10.26481/dis.20101119jg

Document Version:

Publisher's PDF, also known as Version of record

Please check the document version of this publication:

- A submitted manuscript is the version of the article upon submission and before peer-review. There can be important differences between the submitted version and the official published version of record.

People interested in the research are advised to contact the author for the final version of the publication, or visit the DOI to the publisher's website.

- The final author version and the galley proof are versions of the publication after peer review.

- The final published version features the final layout of the paper including the volume, issue and page numbers.

Link to publication

\footnotetext{
General rights rights.

- You may freely distribute the URL identifying the publication in the public portal. please follow below link for the End User Agreement:

www.umlib.nl/taverne-license

Take down policy

If you believe that this document breaches copyright please contact us at:

repository@maastrichtuniversity.nl

providing details and we will investigate your claim.
}

Copyright and moral rights for the publications made accessible in the public portal are retained by the authors and/or other copyright owners and it is a condition of accessing publications that users recognise and abide by the legal requirements associated with these

- Users may download and print one copy of any publication from the public portal for the purpose of private study or research.

- You may not further distribute the material or use it for any profit-making activity or commercial gain

If the publication is distributed under the terms of Article $25 \mathrm{fa}$ of the Dutch Copyright Act, indicated by the "Taverne" license above, 


\section{Influence of micro-environments on pre-school children's energy balance- related behaviours and weight status}

Jessica Sophia Gubbels 


\section{Colophon}

Cover design: Bilbo Schickenberg

Picture cover: Personal archive fam. Gubbels

Printed by: Datawyse

(C) J.S. Gubbels, Margraten, 2010

ISBN: 9789052789958

The studies presented in this dissertation were performed within NUTRIM School for Nutrition, Toxicology and Metabolism.

The research by Jessica Gubbels for the studies presented in this dissertation, is financed by NUTRIM School for Nutrition, Toxicology and Metabolism. Financial support for the KOALA Birth Cohort Study's conception and follow-up up to age 5 was provided by FrieslandCampina, the Dutch Sugar Bureau, University Hospital Maastricht, the Dutch Ministry of Economic Affairs, the Triodos Foundation, and funds unrelated to commercial organizations (Iona Foundation, Phoenix Foundation, Foundation for the Advancement of Heilpedagogiek), all in the Netherlands. TNO Quality of Life particpated in the Lifestyle and Weight project of the KOALA Birth Cohort Study. 


\title{
Influence of micro-environments on pre-school children's energy balance- related behaviours and weight status
}

\author{
PROEFSCHRIFT \\ ter verkrijging van de graad doctor \\ aan de Universiteit Maastricht, \\ op gezag van de Rector Magnificus, \\ Prof. mr. G.P.M.F. Mols, \\ volgens besluit van het College van Decanen, \\ in het openbaar te verdedigen \\ op vrijdag 19 november 2010 om 14:00 uur \\ door \\ Jessica Sophia Gubbels
}

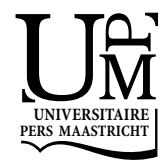




\section{Promotor}

Prof. dr. N.K. de Vries

\section{Copromotores}

Dr. S.P.J. Kremers

Dr. A. Stafleu (TNO Kwaliteit van Leven, Zeist)

Dr. C.T.M.C.N. Thijs

\section{Beoordelingscommissie}

Prof. dr. R.A. Knibbe (voorzitter)

Prof. dr. ir. P.A. van den Brandt

Prof. dr. ir. J. Brug (Erasmus Medisch Centrum, Rotterdam)

Dr. G. Cardon (Universiteit Gent, Gent)

Dr. J. de Nooijer 


\section{Content}

$\begin{array}{lll}\text { Chapter } 1 \text { General introduction } & 7\end{array}$

$\begin{array}{ll}\text { Part 1: Home environment } & 17\end{array}$

Chapter 2 Association of breast-feeding and feeding on demand 19

with child weight status up to 4 years

Chapter 3 Clustering of dietary intake and sedentary behaviour in 31 2-year-old children

Chapter 4 Diet-related restrictive parenting practices. Impact on 41 dietary intake of 2-year-old children and interactions with child characteristics

Chapter 5 Impact of parenting practices on children's dietary intake, physical activity and body mass index: the KOALA Birth Cohort Study

Part 2: Child-care environment

Chapter 6 Child-care use and the association with body mass Index and overweight in children from 7 months to 2 years of age

Chapter 7 Child-care environment and dietary intake of 2- and 3-year-old children

Chapter 8 Interaction between environmental factors determining 99 physical activity of children at child-care

Chapter 9 General discussion

References

Summary

Samenvatting

Dankwoord

Publication list 

Chapter 1: General Introduction 


\section{Childhood overweight}

Overweight and obesity have reached epidemic proportions, both in adults and children (World Health Organization, 2004). Childhood obesity is recognized as one of the most serious public health challenges of the $21^{\text {st }}$ century (World Health Organization, 2010). Globally, at least 20 million children under the age of five were estimated to be overweight in 2005 (World Health Organization, 2006). In 2010, the estimated number of overweight children under five years had risen to 42 million (World Health Organization, 2010), illustrating the alarmingly increasing prevalence rates. Overweight and obesity are problems that also affect Dutch children. Overweight prevalence has more than doubled in young children in the Netherlands between 1980 en 2003: overweight and obesity increased from $5.2 \%$ to $12.8 \%$ in 5 -year-old Dutch boys, and from $8.6 \%$ to $17.5 \%$ in girls of this age (Van den Hurk, van Dommelen, van Buuren, Verkerk, \& Hirasing, 2007).

Overweight and obesity are major risk factors for several chronic conditions such as cardiovascular diseases and type 2 diabetes mellitus (World Health Organization, 2004). Moreover, childhood overweight and obesity are known to track into adulthood, meaning that overweight and obese children often develop into overweight and obese adults (Singh, Mulder, Twisk, van Mechelen, \& Chinapaw, 2008). More specifically, the pre-school age (2-6 years) has been indicated as the most critical growth period for adult overweight (De Kroon, Renders, Van Wouwe, Van Buuren, \& Hirasing, 2010). Treatment has been shown to have poor results for children already suffering from obesity (Ben-Sefer, BenNatan, \& Ehrenfeld, 2009). Early prevention is therefore essential to effectively counteract the obesity epidemic (Kremers, Visscher, Brug et al., 2005).

\section{Energy balance-related behaviours}

Weight gain, ultimately leading to overweight, is the result of a positive energy balance in which energy intake exceeds energy expenditure (World Health Organization, 2004). Behaviours that may influence the energy balance (i.e., dietary intake and physical activity) have been referred to as 'energy balance-related behaviours', or EBRBs (Kremers, Visscher, Seidell, van Mechelen, \& Brug, 2005). 'Obesogenic' behaviours, are EBRBs that may lead to a positive energy balance, and ultimately to obesity (Swinburn, Egger, \& Raza, 1999). In children and adolescents, the most important obesogenic EBRBs include consumption of energydense foods, frequent sedentary behaviour and low levels of physical activity (Rennie, Johnson, \& Jebb, 2005). Since energy balance-related habits are formed at early life stages (Reilly et al., 2004; Savage, Fisher, \& Birch, 2007) and track into later life (Kelder, Perry, Klepp, \& Lytle, 1994), we must increase our understanding of the origin and development of EBRBs in children. The current dissertation will therefore aim to examine the influence of the environment in the development of 
EBRBs. This will enable us to develop evidence-based interventions to prevent overweight development in young children.

\section{Diet}

One of the main risk factors for overweight in childhood is an unhealthy diet (Rennie et al., 2005). It is worrying that unhealthy eating habits often find their origin in early childhood (Savage et al., 2007), and track into later life (Kelder et al., 1994).

The first encounter with food in infancy is drinking milk. Many studies have indicated a protective dose-response relationship of breast-feeding against overweight and obesity development, compared to formula feeding (e.g., Arenz, Ruckerl, Koletzko, \& von Kries, 2004; Harder, Bergmann, Kallischnigg, \& Plagemann, 2005; Owen, Martin, Whincup, Smith, \& Cook, 2005).

Between the ages of six months and two year, children's dietary intake shifts from mainly milk to solid food intake (World Health Organization, 2009). The child's intake pattern becomes much more complex and diverse at this point (World Health Organization, 2009). After this shift to solid foods, specific dietary behaviours that put a child at risk for becoming overweight are a high consumption of energy-dense foods such as snacks and fast foods, a high consumption of sugarsweetened beverages, large portion sizes and eating away from home (Rennie et al., 2005).

\section{Physical Activity}

Low levels of physical activity are among the most important causes of childhood overweight (Rennie et al., 2005). Physical activity habits are formed in early childhood (Reilly et al., 2004), and just as dietary habits they often track into later childhood, adolescence and adulthood (Kelder et al., 1994).

In addition to the importance of a lack of physical activity in obesity development, sedentary behaviour is also an important risk factor (Rennie et al., 2005). Sedentary behaviour includes television viewing, video gaming and computer use. Not only do these behaviours displace physical activity, they are also associated with unhealthy eating habits, posing a double risk on children (Rennie et al., 2005).

\section{Clustering}

Above, some of the most important behavioural determinants of overweight and obesity are described. Note, however, that it is not single behavioural acts, but combinations of multiple risk behaviours and the complex interplay between them that are expected to lead to excess weight gain (Rennie et al., 2005). Several studies 
have examined the co-occurrence, or 'clustering', of EBRBs in general, or more specifically of obesogenic behaviours. Clustering is defined as a combination of behaviours which is more prevalent than one would expect based on the prevalence of the separate behaviours (Schuit, van Loon, Tijhuis, \& Ocke, 2002). The potential synergy between EBRBs could be used in obesity prevention interventions, applying an integrated approach in which multiple EBRBs are addressed simultaneously. Moreover, determining which children show obesogenic clustering, helps us to identify high risk group that may be targeted in preventive interventions.

Various EBRBs have been found to cluster in children. Within dietary intake behaviour, unhealthy consumption behaviours (e.g., snack or soft drink intake) have been found to cluster, and to be inversely associated with healthy dietary intake behaviours (e.g., fruit and vegetable intake; Knol, Haughton, \& Fitzhugh, 2005; Kremers, De Bruin, Schaalma, \& Brug, 2004; Northstone \& Emmett, 2005; Striegel-Moore et al., 2006). Within the behavioural domain of activity behaviour, television viewing and computer use have been found to be negatively related with physical activity, and positively with each other (DuRant, Baranowski, Johnson, \& Thompson, 1994; Marshall, Biddle, Gorely, Cameron, \& Murdey, 2004; Nelson, Gordon-Larsen, Adair, \& Popkin, 2005; Robinson et al., 1993). However, there is also evidence of clustering across the behavioural domains of dietary intake and physical activity. Negative associations have been found between television viewing and consumption of healthy foods such as vegetables and fruit (Boynton-Jarrett et al., 2003; Utter, Neumark-Sztainer, Jeffery, \& Story, 2003). Positive associations have been found between unhealthy food intake (e.g., intake of snacks, soft drinks and fat) on the one hand, and television viewing and computer use on the other (Boynton-Jarrett et al., 2003; Coon, Goldberg, Rogers, \& Tucker, 2001; Lioret, Touvier, Lafay, Volatier, \& Maire, 2008; Utter et al., 2003). Physical activity was found to be inversely related to an unhealthy diet (NeumarkSztainer et al., 1997), and positively to healthy food intake (Kremers et al., 2004; Lioret et al., 2008; Neumark-Sztainer et al., 1997).

\section{Environment}

\section{Ecological perspective}

EBRBs have been hypothesized to be influenced by multiple levels and types of environment. Swinburn and colleagues (1999) distinguish four types of environment: the physical environment (i.e., what is available), the sociocultural environment (i.e., beliefs, attitudes and values related to EBRBs), the economic environment (i.e., the costs related to EBRBs) and the political environment (i.e., the rules related to EBRBs). Moreover, they distinguish two levels of environmental influences: the micro level, i.e., the direct setting in which the behaviour is executed, and the macro level, i.e., the higher level sector environment (Swinburn et 
al., 1999). The current dissertation focuses on the micro level environment in which children's EBRBs take place.

The ecological 'systems' view of environmental influences on behaviour (Friedman \& Wachs, 1999; Spence \& Lee, 2003; Wachs, 1992) assumes that within the micro level, physical environmental factors interact with the actors that operate within this setting (i.e., the social environment). In line with this, higher order variables, which are more distal to the individual behaviour, have been hypothesized to moderate the influence of the more proximal, lower order variables (Friedman \& Wachs, 1999). A moderator variable is a variable that affects the direction and/or strength of the relation between an independent variable and a dependent variable (Baron \& Kenny, 1986). In the case of the ecological framework, this means that the influence of proximal environmental variables on behaviour is dependent on the distal environment. Other terms that are often used to indicate such moderation are effect-modification and interaction.

In addition, it has been theorized that the influence of the environment on EBRBs behaviour can be moderated by several individual factors, including demographic characteristics such as age and gender (Kremers et al., 2006; Kremers, Schaalma, Meertens, van Mechelen, \& Kok, 2008).

\section{Home environment}

Young children spend the greater part of their time at home, with their parents. Parents can have a strong influence on their child's health behaviour, including their EBRBs. For instance, parents control the availability of, and the exposure to food and physical activity opportunities; parents act as role models; and parents can provide their child with support and structure. Furthermore, they can use specific parenting practices (Golan \& Crow, 2004), which are content-specific acts of parenting (Darling \& Steinberg, 1993), such as rules about dietary intake or physical activity.

\section{Child-care environment}

In addition to parental care, young children nowadays spend increasing amounts of time in child-care. In Europe, a quarter of the infants and over three quarters of the children up to school age attend some form of child-care or education facilities (Blackburn, 2006; Eurostat, 2008). Child-care has been indicated as an important setting for prevention of overweight and obesity development in young children (Story, Kaphingst, \& French, 2006). This importance is apparent in the recommendation that a child who spends a full day (i.e., eight hours or more) in child-care should consume one half to two-thirds of his or her daily dietary intake in child-care (American Dietetic Association, 1994). Furthermore, a recent review of correlates of preschool children's physical activity showed that the preschool a child attends is statistically significantly associated with the child's physical activity (Hinkley, Crawford, Salmon, Okely, \& Hesketh, 2008). The role of staff members 
at child-care is comparable to the role that parents have at home. For instance, just like parents apply certain parenting practices to influence their child's dietary intake and physical activity, child-care staff also applies specific supervision practices.

Application of the ecological perspective in children's micro settings

Figure 1.1 shows a graphical representation of the application of the ecological perspective in the current study. This is also the research framework for the current dissertation. In line with the ecological perspective described above, it has been signalled that there is an urgent need to know whether the effects of parenting are similar across distinct groups of children (e.g., Faith, Berkowitz et al., 2004). Several child characteristics have been found to moderate the effects of diet-related parenting practices. For instance, restriction and control have been found to have stronger undesirable effects on dietary intake of girls than of boys (e.g., Arredondo et al., 2006; Faith, Scanlon, Birch, Francis, \& Sherry, 2004), but studies regarding moderation of gender in the relationship between restriction and weight status have shown mixed results (Faith, Scanlon et al., 2004).

In line with the findings regarding parental influences, the influence of the child-care environment on children's EBRBs has also been found to be moderated by child characteristics. For example, age has been found to moderate the effect of the physical child-care environment on physical activity (Ridgers, Stratton, Fairclough, \& Twisk, 2007a), and indications were found that the social preschool environment has a stronger influence on girls than on boys (Cardon, Van Cauwenberghe, Labarque, Haerens, \& De Bourdeaudhuij, 2008).

\begin{abstract}
Aims
The aim of the current project was to examine the direct and interactive effects of specific features of micro environments in which young children up to 5 years of age spend their time, on these children's EBRBs (i.e., dietary intake and physical activity) and weight status. The micro environments that were examined were the home environment and the child-care environment, as these are the settings in which children under the age of 5 years spend most of their time.
\end{abstract}




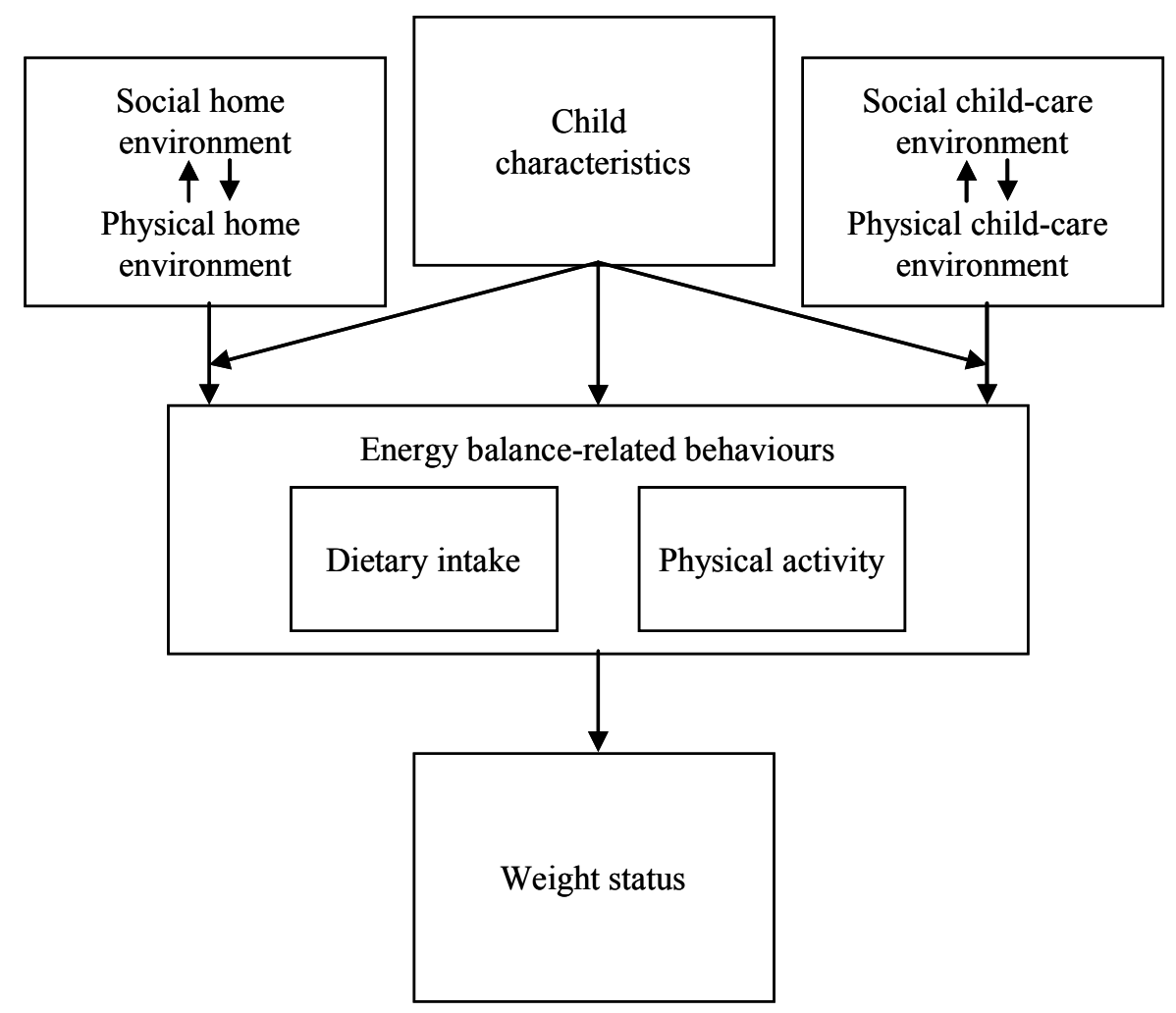

Figure 1.1: Graphical representation of the research framework

\section{Study design}

Two different sets of data regarding young children were used for the studies that are described in the current dissertation. Their designs will be described shortly below.

First of all, data from the KOALA Birth Cohort Study (the Netherlands) were used. The KOALA Cohort is a prospective cohort study which was started in the year 2000. KOALA is the Dutch acronym for Child, Parent and health: Lifestyle and Genetic constitution (Kind, Ouders en gezondheid: Aandacht voor Leefstijl en Aanleg). Healthy pregnant women participating in a study of the development of pregnancy-related pelvic girdle pain were recruited for the study (conventional recruitment group). In addition, women were recruited through 'alternative' recruitment channels (e.g., anthroposophist midwives and general practitioners, Steiner schools and organic food shops; Kummeling et al., 2005). This alternative recruitment group (17.3\%) had alternative lifestyles in terms of for instance dietary habits (e.g., choice of organic foods) or child rearing. All participants signed informed consent, and ethical approval was obtained from the Maastricht 
University/University Hospital Maastricht medical ethics committee. In total, 2834 women participated, and completed questionnaires during pregnancy, and regularly after their child was born (at ages 3 and 7 months, and 1,2 and 5 years). These questionnaires assessed various parental and child background characteristics, such as parental educational level, age, working hours and marital status, and child gender and birth weight. In addition, anthropometric data were assessed via the parents, including child and parent weight and height at several time points. The questionnaires furthermore assessed EBRBs of the children, such as the time spent in different activities (e.g., playing outside, television viewing), and their dietary intake at different ages. Finally, the questionnaires assessed various other components of the child's life, such as child-care and school use and parental use of energy balance-related parenting practices. In addition to the questionnaires, there were several subgroups within the cohort that received additional measures, such as home visits at which height and weight were assessed. The KOALA dataset was used in the Chapters 2 to 6 .

In addition to the KOALA data, a second dataset was obtained through observations at nine child-care centres in Maastricht, the Netherlands. These childcare centres were all part of the coordinating organization Maatwerk In Kinderopvang (MIK), i.e., 'custom-made child-care'. All nine child-care centres were visited three times: once for an interview with the centre manager and a tour around the facility, and twice for observations. The interviews and observations were performed in May and June of 2008. Two trained observers performed the observations. At the observation days, children were randomly selected for observations. Physical activity intensity and environmental features were observed by a momentary-sampling procedure. In total, physical activity of 10 children was observed per centre, per day. In addition to the children's physical activity, dietary intake during the eating moments was observed. Five children were observed per centre, per eating moment. The eating moments that were observed at each childcare centre were the morning snack, the lunch break, and the afternoon snack. Trained observers assessed dietary intake, as well as various environmental features during the meal, such as staff modelling behaviour. Each child-care centre was visited during one morning and one afternoon on 2 separate days, with at least one week between the 2 observation days. In total, physical activity was observed for 175 children, and dietary intake was observed for 130 children. The observational child-care studies are reported in the Chapters 7 and 8 .

\section{Outline}

The current dissertation consists of two parts, focussing on the two main micro environments that infants and pre-school children up to 5 years encounter, i.e., the home environment and the child-care environment. The outline of these parts and the specific chapters are described below. 
The first part of this dissertation discusses the influence of the home environment on children's EBRBs and weight status. Chapter 2 describes the influence of breast-feeding duration and the feeding pattern parents apply, on the development of childhood overweight. Chapter 3 discusses clustering of EBRBs in 2-year-old children, and the influence of parental characteristics on these clusters. Chapter 4 describes the influence of diet-related parenting practices on 2-year-olds' dietary intake. Chapter 5 discusses the influence of energy balance-related parenting practices on 5-year-olds' physical activity and dietary intake, and their subsequent BMI. Chapters 4 and 5 both take into account the interaction between child and parent, by applying moderation analyses.

The second part discusses the influence of the child-care environment on children's EBRBs and weight status. Chapter 6 describes the association between child-care use and BMI and overweight, as well as parental predictors of child-care use. Chapter 7 examines dietary intake at child-care and its association with childcare staff behaviour. Chapter 8 investigates the direct and interactive association of both the social and the physical child-care environment with children's physical activity levels at child-care.

Finally, Chapter 9 provides an overview and a general discussion of all the chapters described above, by discussing the most important micro-environmental determinants of young children's physical activity and dietary intake behaviour. Furthermore, it will discuss the practical and theoretical implications of the findings of the current project. 



\section{Part 1: Home environment}





\section{Chapter 2: Association of breast-feeding and feeding on demand with child weight status up to 4 years}

This chapter has been accepted for publication as: Gubbels, J.S., Thijs, C., Stafleu, A., van Buuren, S. \& Kremers, S.P.J. (In Press). Association of breastfeeding and feeding on demand with child weight status up to 4 years. International Journal of Pediatric Obesity. 


\begin{abstract}
Objectives: The mechanisms underlying the protective effect of breast-feeding on the development of childhood overweight are unclear. This study examines the association of breast-feeding with weight gain in the first year, and BMI and overweight up to 4 years. In addition, we examine possible mechanisms of this effect (i.e., feeding pattern, eating style, unhealthy snacking behaviour).

Methods: Data originated from the KOALA Birth Cohort Study ( $\mathrm{N}=2834)$. Questionnaires assessed breast-feeding duration up to 12 months, feeding pattern (i.e., feeding on demand or feeding to schedule) at 3 months, BMI at 1, 2 and 4 years, eating style (e.g., slow eating) at age 1, and unhealthy snacking at age 2 . Linear and logistic regression analyses were used to examine the association of breast-feeding and feeding pattern with eating style, unhealthy snacking, BMI zscores and overweight.

Results: Each additional month of breast-feeding was associated with less weight gain in the first year (regression coefficient $\mathrm{B}=-37.6$ grams, $\mathrm{p}<.001$ ), a lower BMI $\mathrm{z}$-score at age $1(\mathrm{~B}=-.02, \mathrm{p}<.01)$, and a lower odds of being overweight at age 1 (odds ratio=.96, $\mathrm{p}<.05$ ). Breast-feeding was associated with fewer unhealthy snacking occasions per week at age $2(B=-.19$ for each month of breast-feeding, $\mathrm{p}<.001$ ), but was unrelated to eating style. Feeding pattern was unrelated to all outcome variables after adjustment for breast-feeding duration.

Conclusions: The study showed a short-term protective effect of breast-feeding against overweight development. Possible mechanisms through which breastfeeding may protect against overweight include less unhealthy snacking behaviour, but not feeding pattern or child's eating style.
\end{abstract}




\section{Introduction}

An unhealthy diet is among the most important determinants of childhood overweight (Rennie et al., 2005). Dietary habits are often formed at a young age, and once established maintained throughout life (Kelder et al., 1994; Singer, Moore, Garrahie, \& Ellison, 1995). The first encounter with food in infancy is drinking milk, and many studies have examined the influence of breast-feeding compared to formula feeding on weight development (Arenz et al., 2004; Harder et al., 2005; Owen et al., 2005). Most indicate that breast-feeding protects against overweight and obesity development (Arenz et al., 2004; Harder et al., 2005; Owen et al., 2005), with a dose-response relationship (Harder et al., 2005). However, some studies reported no effect of breast-feeding on later overweight (Hediger, Overpeck, Kuczmarski, \& Ruan, 2001; Huus, Ludvigsson, Enskar, \& Ludvigsson, 2008; Neutzling et al., 2009).

The mechanisms through which breast-feeding might protect against overweight development are unclear, although both behavioural and physiological mechanisms have been proposed to play a role (Arenz et al., 2004; Bartok \& Ventura, 2009; Harder et al., 2005). One of the behavioural mechanisms linked to the protective effect of breast-feeding regards the feeding pattern and practices used by mothers (Gillman et al., 2001). The 'Start Healthy Feeding Guidelines for Infants and Toddlers' of the American Dietetic Association recommend responsive parenting, recognizing and appropriately responding to infant hunger and satiety cues, including feeding on demand (Butte et al., 2004). Dutch guidelines are in line with this (Voedingscentrum, 2008). Some authors suggest that breast-fed children may have more control over the amount they consume, as breast-feeding mothers can only rely on infants' signals for frequency and volume of feedings. Formula-fed children are more often fed to a fixed schedule, with a fixed volume to drink, possibly leading to overconsumption (Gillman et al., 2001). However, although breast-fed children are more likely to be fed on demand and formula-fed children more often to schedule (Gillman et al., 2001), these combinations are not fixed. Bartok and Ventura (2009) recently called for examination of interactive effects of infant feeding components.

Other researchers have suggested a negative influence of feeding on demand on weight development. Erlanson-Albertsson and Zetterstrom (2005) suggested that not adhering to a fixed feeding schedule when breast-feeding, but feeding on demand instead, would lead to overweight. The mechanism claimed to be responsible for this hypothetically increased risk is that children fed on demand consume smaller volumes several times throughout the day, leading to a habit of snacking in later life. This in turn would favour obesity development (ErlansonAlbertsson \& Zetterstrom, 2005). They also argue that mothers feeding on demand might soothe children crying for reasons other than hunger, by providing them with milk, leading to overfeeding and overweight (Erlanson-Albertsson \& Zetterstrom, 
2006). Feeding in response to emotional cues is indeed considered undesirable (Savage et al., 2007). The hypothesis of Erlanson-Albertsson and Zetterstrom has received critique (Aarts \& Greiner, 2006), as feeding on demand is considered to be important for establishing a balanced nutritional homeostasis (Marchini, Persson, Berggren, \& Hagenas, 1998).

We examined effects of both the type of infant feeding (breast-feeding versus formula feeding), and the feeding pattern (feeding to a fixed schedule versus feeding on the child's demand), on weight gain, body mass index (BMI) and overweight in early childhood. We explored the interactive effect of feeding pattern and type, to examine the possibility that their combination is responsible for the association with weight status. We also examined the relationship of infant feeding with children's eating style and unhealthy snacking behaviour at later ages, to elucidate the possible overweight-related behavioural consequences of feeding types and patterns.

\section{Methods}

\section{Respondents and procedure}

The Dutch KOALA Birth Cohort Study is a prospective cohort study which started in the year 2000. The KOALA cohort was initially focused on the aetiology of atopic diseases (Kummeling et al., 2005). Pregnant women were recruited from a cohort of healthy women from the general population, recruited by midwives for a study of the aetiology of pregnancy-related pelvic girdle pain (conventional recruitment group; Bastiaanssen et al., 2005; Kummeling et al., 2005). A total of $38.6 \%$ of the approached mothers agreed to participate $(\mathrm{N}=2343)$. In addition, healthy pregnant women were recruited through 'alternative lifestyle' recruitment channels (e.g., anthroposophist midwives, organic food shops; Kummeling et al., 2005). This alternative recruitment group $(\mathrm{N}=491)$ had an alternative lifestyle in terms of aspects like dietary habits (e.g., using organic foods). All 2834 participating mothers signed informed consent, and ethical approval was obtained from the Maastricht University/University Hospital Maastricht medical ethics committee. Ten children were excluded because of congenital defects (e.g., Down's syndrome), leaving 2824 children for analyses. Questionnaires at the child's ages of $3(\mathrm{~N}=2645)$ and 7 months $(\mathrm{N}=2606)$, and at $1(\mathrm{~N}=2557), 2(\mathrm{~N}=2569)$ and 4 years $(\mathrm{N}=2037)$, were used to assess various characteristics of the children and their parents. Questionnaires at all 5 ages were returned for a total of 1863 children (66.0\%). Mothers who returned all five questionnaires had children with a slightly higher birth weight (3529 vs. $3460 \mathrm{~g} ; \mathrm{p}<.01)$. There was no significant difference between these groups in maternal pre-pregnancy BMI $\left(23.6 \mathrm{~kg} / \mathrm{m}^{2}\right.$ in both groups; $\mathrm{p}>.05$ ). 


\section{Child feeding}

The 3-months questionnaire asked parents whether the child had received breastmilk, formula feeding or a combination of both during the first three months of life. The questionnaires at 7 and 12 months asked parents to indicate the duration of breast-feeding in the period between the questionnaires (including non-exclusive breast-feeding). These questions were combined into one measure of breast-feeding duration in months. No breast-feeding in the first three months was coded 0 ; partly breast-feeding and partly formula feeding in the first three months was coded as the midpoint value of the category, i.e., 1.5 months. For children that were still being breast-fed at 3 months, the breast-feeding duration was derived from the questionnaire at 7 months. For children who were still being breast-fed at 7 months, the duration was derived from the questionnaire at 12 months.

The feeding pattern was assessed in the 3 months questionnaire, asking: 'How did you try to feed your baby during the last week?' Answering options were 'When the baby asked for it', which we called 'feeding on demand'; 'According to a fixed schedule', called 'feeding to schedule'; and 'Both', called 'mixed feeding'.

\section{Child's eating style and unhealthy snacking behaviour}

The child's eating style at 1 year was assessed by asking parents how they would describe their child's eating behaviour, using 7 eating style characterizations: 'slow', 'eager', 'fast', 'with reluctance', 'with pleasure', 'doesn't like many things' and 'likes many things'. Each of these items was considered as a separate eating style characterization, and could either apply or not. Multiple eating style characterizations could therefore be applicable simultaneously (e.g., eating both slowly and with pleasure).

Unhealthy snacking behaviour at age 2 was assessed by asking parents how often their child consumed the following types of snacks: chocolate, cookies and pastry, chips and other savoury snacks, and sweets. Response categories for each type were 'never', 'less than once a week', '1-3 times a week', '4-6 times a week', 'once a day', 'twice a day', ' 3 times a day' and 'more than 3 times a day'. The intake was recoded into weekly intake frequency (e.g., once a day was recoded into 7 times a week). For response categories that comprised a certain range of frequencies, the midpoint value of this range was used (e.g., 1-3 times was translated into 2 times a week). Intake of the various snacks (i.e., chocolate, cookies and pastry, chips and other savoury snacks, sweets) was then summed, resulting in one measure of total unhealthy snack intake frequency. Portion size of the snacks was not assessed. 
Parents were asked to report their child's birth weight, as well as height and weight at 1, 2 and 4 years. Weight gain in the first 12 months of life was calculated by subtracting birth weight from weight at age 12 months (standardized for age; both in grams). BMI (i.e., weight $(\mathrm{kg}) /(\text { height }(\mathrm{m}))^{2}$ ) was calculated at ages 1,2 and 4 and converted to z-scores, using data from the national reference population (Fredriks, van Buuren, Wit, \& Verloove-Vanhorick, 2000). BMI z-scores indicate by how many standard deviations a child differs from the median BMI of the reference population for his/her age. We classified children with a BMI z-score above the $85^{\text {th }}$ percentile as overweight (Barlow, 2007), including those who were obese.

\section{Other parental and child characteristics}

Mothers were asked to indicate their own height and weight before they got pregnant, and their pre-pregnancy BMI was calculated. Maternal age at delivery and smoking during pregnancy were also assessed, as well as the child's gender.

\section{Data analyses}

The distribution of demographic background variables, breast-feeding duration and feeding pattern were explored, after which regression analyses were performed. Children fed according to a mixed feeding pattern (on demand as well as to schedule) were excluded from all regression analyses $(\mathrm{N}=216)$. Missing values were excluded pair-wise. The influence of breast-feeding duration (in months) and feeding pattern on eating style at 1 year was explored using binary logistic regression analyses with each of the 7 eating style characterizations (applicable/non-applicable) as dependent variables. The influence on unhealthy snack intake at age 2 was explored using linear regression analyses. The analyses were adjusted for the influence of several potential confounders: child gender and birth weight, maternal age and pre-pregnancy BMI, maternal smoking during pregnancy (yes/no) and recruitment group (alternative vs. conventional). To test for potential effect-modification (i.e., moderation) of feeding pattern in the relationship between breast-feeding duration and eating style, an interaction term was included in the second step of the analyses. Stratified regression analyses were performed in case of significant interaction terms.

The influence of breast-feeding and feeding pattern on weight gain in the first year and on BMI z-score and overweight (yes/no) at ages 1, 2, and 4 years was examined in a similar manner, using linear and logistic regression. The feeding variables and covariates were included in the first step, the interaction between feeding pattern and breast-feeding duration in a separate step. Stratified regression 
analyses were performed for significant interaction terms. P-values $<.05$ were considered statistically significant.

\section{Results}

A little over half $(51.2 \%)$ of the children were male, and mean birth weight was $3506 \mathrm{~g}(\mathrm{SD}=511)$. Mean maternal pre-pregnancy BMI was $23.6 \mathrm{~kg} / \mathrm{m}^{2}(\mathrm{SD}=4.0)$ and $7.3 \%$ of the mothers smoked during pregnancy. Mean maternal age at delivery was 32 years $(\mathrm{SD}=4)$.

Children gained an average of $6288 \mathrm{~g}(\mathrm{SD}=958)$ during their first year. Mean BMI z-score at 1 year was -.01 ( $\mathrm{SD}=1.03)$, and 365 children $(15.1 \%)$ were overweight (including those who were obese). Mean BMI z-score at age 2 was -.04 $(\mathrm{SD}=1.05)$, and 340 children $(14.5 \%)$ were overweight. A total of 170 children $(8.9 \%)$ were overweight at age 4 , with a mean BMI z-score of -.26 ( $\mathrm{SD}=.99)$.

\section{Breast-feeding duration and feeding patterns}

The vast majority $(80.2 \%)$ of the mothers had initiated breast-feeding. Table 2.1 shows the feeding patterns used by mothers in different categories of breast-feeding duration. There was a strong association between feeding pattern and duration of breast-feeding (Pearson $\mathrm{Chi}^{2}=334.6, \mathrm{p}<.001$ ). Of the mothers who never breast-fed their child (i.e., exclusively formula feeding), about three quarters $(78.0 \%)$ fed to schedule. By contrast, slightly over half of those who breastfed for 4 months or more did so on demand.

Table 2.1: Feeding pattern according to breast-feeding duration $(\mathrm{N}=2634)$

\begin{tabular}{lccccc}
\hline & & \multicolumn{3}{c}{ Feeding pattern N $(\%)^{a}$} & $\mathrm{~N}^{a}(\%)^{b}$ \\
\cline { 3 - 5 } & & On demand & To schedule & Mixed & Total \\
\hline \multirow{2}{*}{ No breast-feeding } & 67 & 319 & 23 & $\mathbf{4 0 9}$ \\
& & $(16.4 \%)$ & $(78.0 \%)$ & $(5.6 \%)$ & $\mathbf{( 1 5 . 5 \% )}$ \\
Breast-feeding & $1-3$ months & 236 & 577 & 65 & $\mathbf{8 7 8}$ \\
duration & & $(26.9 \%)$ & $(65.7 \%)$ & $(7.4 \%)$ & $\mathbf{( 3 3 . 3 \% )}$ \\
& $4-6$ months & 230 & 171 & 40 & $\mathbf{4 4 1}$ \\
& & $(52.1 \%)$ & $(38.8 \%)$ & $(9.1 \%)$ & $\mathbf{( 1 6 . 7 \% )}$ \\
& & 508 & 310 & 88 & $\mathbf{9 0 6}$ \\
& & $(56.1 \%)$ & $(34.2 \%)$ & $(9.7 \%)$ & $\mathbf{( 3 4 . 4 \% )}$
\end{tabular}

$\bar{a} \%$ for each feeding pattern category are calculated $\overline{\text { horizontally (as proportion of the breast-feeding }}$ duration category; for N's see final column).

${ }^{b} \%$ for each breast-feeding duration category are calculated vertically (as proportion of the total sample; $N=2634$ ). 
Table 2.2 shows the association of feeding pattern and breast-feeding duration with children's eating style at age 1 and unhealthy snacking behaviour at age 2 . Feeding pattern was unrelated to eating style and unhealthy snacking, and breast-feeding duration was not associated with eating style either. However, each additional month of breast-feeding duration was associated with .19 fewer unhealthy snacking occasions a week at age $2(p<.001)$. Feeding pattern and breast-feeding duration did not interact in determining unhealthy snacking behaviour or eating style (tests for statistical interaction $\mathrm{p}<.05)$.

\section{Association of feeding with weight gain, BMI and overweight}

Table 2.3 shows the association of feeding pattern and breast-feeding duration with weight development. The influence of feeding pattern was adjusted for breastfeeding (and vice versa). Feeding pattern was unrelated to weight gain, BMI or overweight. Breast-feeding duration was negatively associated with weight gain: children gained, on average, $37.6 \mathrm{~g}$ less in their first year for each additional month of breast-feeding $(\mathrm{p}<.001)$. Breast-feeding duration was also slightly negatively associated with BMI z-score at age 1 , decreasing by .02 for each additional month of breast-feeding $(\mathrm{p}<.01)$. Consistent with this finding, each additional month of breast-feeding significantly decreased the odds of being overweight at age 1 $(\mathrm{OR}=.96,95 \%-\mathrm{CI}=.93-1.00)$. No associations between breast-feeding and BMI or overweight were found at ages above 1 year, and breast-feeding and feeding pattern did not interact in determining weight gain, BMI, or overweight.

\section{Discussion}

The current study examined the association of infant feeding type and pattern with weight development in early childhood. Breast-feeding duration was found to be inversely associated with weight gain in the first year of life, BMI, and overweight at age 1. This confirms conclusions of previous reviews regarding the (dosedependent) protective effect of breast-feeding against overweight (Arenz et al., 2004; Harder et al., 2005; Owen et al., 2005). We failed to find effects on BMI or overweight beyond the age of 1 year. 


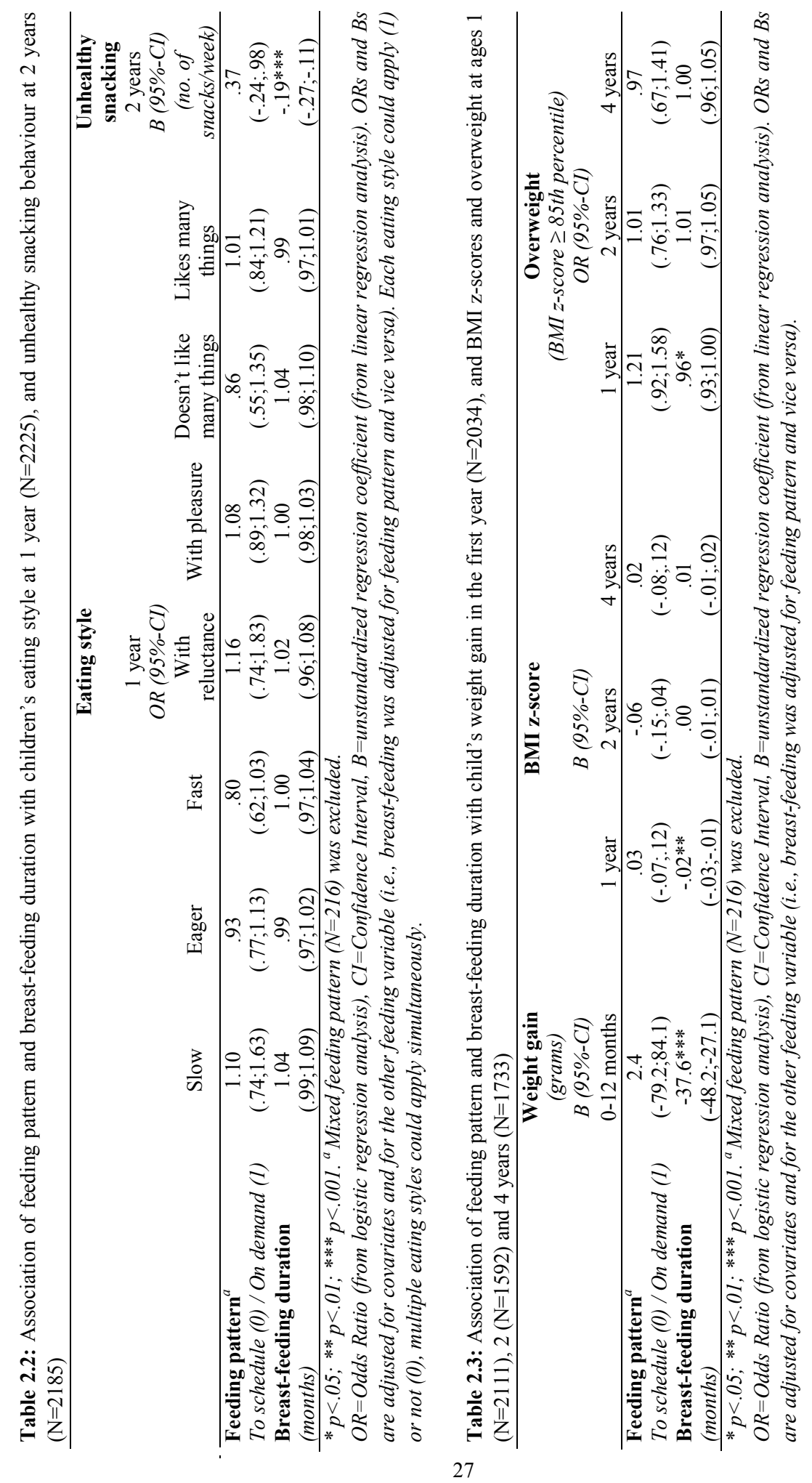


One of the mechanisms through which breast-feeding has been proposed to protect against overweight is the feeding pattern, suggesting that breast-fed children are more often fed on demand, which is postulated to be preferable to feeding to schedule because a fixed feeding schedule could disturb a child's internal regulation of satiety and hunger (Gillman et al., 2001). In line with this, breast-feeding has previously been found to be associated with less maternal controlling behaviour at the age of 1 year (Taveras et al., 2004), which partly explained the association between breast-feeding and overweight (Taveras et al., 2006). The current study shows that children breast-fed for more than 3 months were indeed mostly fed on demand, whereas formula-fed children were more often fed to schedule. However, children breast-fed for less than 3 months were also most often fed to schedule. This probably results from the way feeding pattern was assessed, by asking parents at infant age 3 months. Mothers who breast-fed up to 3 months switched to formula feeding somewhere in those first three months. They were therefore formula feeding, and thus probably feeding to schedule, at the time of the questionnaire. An alternative explanation could be that mothers who breast-fed to schedule were less successful than those breast-feeding on demand, and therefore stopped breastfeeding at an earlier age. In addition, mothers who explicitly choose to breast-feed for a longer time, might also explicitly choose to feed on demand, as both are recommended by professionals (Butte et al., 2004; Voedingscentrum, 2008), while mothers who breast-feed for a shorter duration might be less conscious in their choice, or less successful in following the recommendations.

Although breast-feeding mothers more often fed on demand, feeding on demand was unrelated to BMI or overweight. This contradicts the suggestion by Gillman et al. (2001) that feeding pattern and its influence on the child's selfregulation of energy intake is the mechanism through which breast-feeding protects against overweight development. On the other hand, the hypothesis of ErlansonAlbertsson and Zetterstrom (2005) that feeding on demand causes overweight was not confirmed either: feeding on demand was not associated with a higher BMI or increased odds of becoming overweight.

Bartok and Ventura (2009) suggested that the interactive effects of the many components of infant feeding are responsible for the association with weight status and that these components are inseparable. We found no interaction between breast-feeding and feeding pattern, which means that there was no moderation (i.e., effect-modification) of feeding pattern in the relationship between breast-feeding and weight status. Thus, the effect of breast-feeding was not dependent on whether the feeding pattern was on demand or to schedule. However, as our analyses were based on a single measurement of feeding pattern, our results do not rule out a dynamic interaction over time.

We further examined possible mechanisms explaining the protective effect of breast-feeding by assessing the association of breast-feeding and feeding pattern with subsequent eating styles and unhealthy snacking. Although breast-feeding was 
unrelated to eating style, breast-fed children consumed significantly fewer unhealthy snacks at age 2, especially with longer breast-feeding duration. Previous research also found that breast-fed children consumed fewer snacks at age 7, although this did not explain the difference in overweight between breast-fed and formula-fed children (Scholtens et al., 2008). Breast-feeding has further been shown to influence children's food preferences (Forestell \& Mennella, 2007; Mennella, Jagnow, \& Beauchamp, 2001), which might explain the inverse association found between breast-feeding and unhealthy snacking. Breast-feeding has also been found to be associated with later parental feeding style and practices (Farrow \& Blissett, 2006; Fisher, Birch, Smiciklas-Wright, \& Picciano, 2000; Taveras et al., 2004), which could indicate that breast-feeding merely reflects parents' global parenting philosophy. Other suggested behavioural mechanisms include children's fruit and vegetable intake (Cooke et al., 2004; Scholtens et al., 2008; Toschke et al., 2002), sweetened beverage intake and added sugar intake (Lande et al., 2004), and sedentary behaviour (Scholtens et al., 2008; Toschke et al., 2002), although most studies did not find significant mediation of these factors in the relationship between breast-feeding and weight development (Scholtens et al., 2008; Toschke et al., 2002). Some suggested that mothers who breast-feed might be more nutritionconscious in general (Kramer et al., 2002). This does not, however, exclude the possibility that maternal nutrition-consciousness can develop or change during early motherhood (with its dietary changes related to the psychophysiology of pregnancy and lactation), as mothers gain more experience with child feeding. The possibility that breast-feeding reflects a general nutrition or lifestyle orientation seems specifically probable in the respondents from the alternative recruitment group in the current study (Kummeling et al., 2005), but all analyses were corrected for recruitment group. Feeding pattern was unrelated to eating style or unhealthy snack intake in the current study. These findings further refute the hypothesis of ErlansonAlbertsson and Zetterstrom (2005).

Note that non-exclusive breast-feeding was included in our measure of breast-feeding duration. Performing the same analyses with exclusive breastfeeding duration could possibly have yielded different results, as exclusivity of breast-feeding has been reported to strengthen the protective effects against overweight (Bartok \& Ventura, 2009). In line with this, introduction to solid foods might confound current findings, as breast-feeding duration and age of introduction to solids have been found te be negatively correlated (see e.g., Burdette, Whitaker, Hall, \& Daniels, 2006). A recent study showed that the effects of breast-feeding on long term BMI, decreased when age of introduction to solid foods was added to the analyses (Schack-Nielsen, Sorensen, Mortensen, \& Michaelsen, 2010). The relationships of solid food introduction and exclusive breast-feeding with infant feeding patterns are unclear, and should be further examined in future research. In addition, we did not assess whether children drank expressed breast-milk from a bottle instead of directly from their mother's breast. Examining the effect of bottle- 
fed breast-milk could be an important step in sorting out the mechanisms behind the effects of breast-feeding on weight status.

Some limitations should be taken into account when interpreting our results. Overweight percentages in this study were relatively low, compared to the reference population (e.g., $8.9 \%$ vs. $15 \%$ at age 4; Fredriks, et al., 2000), which perhaps limits the generalizability of the current findings. In addition, all data, including weight, height and feeding variables, were reported by parents and could therefore be biased. Previous research in the Netherlands has shown that parental reports of weight and height showed only small differences from measured weight and height. However parents especially tended to underestimate overweight children's weight (Scholtens et al., 2007). Possibly this also resulted in underestimation of children's BMI and overweight prevalence in the current study. However, the questionnaires at ages 1 and 2 years were completed shortly after visits to infant welfare centres (typically scheduled around 11 and 24 months, respectively), and parents were asked to report the results of anthropometric measurements taken there. Furthermore, data were gathered longitudinally, which decreases the probability of recall bias. The assessment of the feeding variables also poses some limitations. Breast-feeding in the first three months was not assessed for each month specifically, but rather as a categorical variable (no breast-feeding, partly breast-feeding or completely breast-feeding). Feeding pattern was assessed only once, at 3 months. Finally, several concepts (e.g., feeding pattern, eating style, snacking behaviour) were assessed with questions that were developed for the current study, and have not been previously validated.

This study showed that breast-feeding had a short-term protective effect against overweight at the age of 1 year. In the longer run, however, no association was found between breast-feeding and BMI or overweight. The present study was, to our knowledge, the first to examine the influence of feeding pattern, and found no significant association between feeding pattern and BMI, overweight, unhealthy snacking, or eating style. The results do support the notion that feeding on demand is an element in mother-child adaptations contributing to successful breast-feeding continuation. Breast-feeding duration was associated with less unhealthy snacking at age 2, which might be one of the behavioural mechanisms through which breastfeeding favourably influences weight development. By contrast, an influence of breast-feeding on eating style does not seem to be a major protective mechanism. We conclude, first, that feeding on demand is not deleterious in terms of eating habit formation and the development of overweight; and second, that breast-feeding is associated with less unhealthy snacking, protecting against overweight development in childhood, and therefore possibly also against later obesity. 


\section{Chapter 3: Clustering of dietary intake and sedentary behaviour in 2-year-old children}

This chapter has been published as: Gubbels, J.S., Kremers, S.P.J., Stafleu, A., Dagnelie, P.C., de Vries, S.I., de Vries, N.K. \& Thijs, C. (2009). Clustering of dietary intake and sedentary behavior in 2-year-old children. Journal of Pediatrics, 155(2), 194-198. 


\begin{abstract}
Objective: To examine clustering of energy balance-related behaviours (EBRBs) in young children. This is crucial because lifestyle habits are formed at an early age and track in later life. This study is the first to examine EBRB clustering in children as young as 2 .

Study design: Cross-sectional data originated from the Child, Parent and Health: Lifestyle and Genetic Constitution (KOALA) Birth Cohort Study. Parents of 2578 2-year-old children completed a questionnaire. Correlation analyses, principal component analyses and linear regression analyses were performed to examine clustering of EBRBs.

Results: We found modest but consistent correlations between EBRBs. Two clusters emerged: a 'sedentary-snacking cluster' and a 'fibre cluster'. Television viewing clustered with computer use and unhealthy dietary behaviours. Children who frequently consumed vegetables also consumed fruit and brown bread more often, and white bread less often. Lower maternal education and maternal obesity were associated with high scores on the sedentary-snacking cluster, whereas higher educational level was associated with high fibre cluster scores.

Conclusions: Obesity-prone behavioural clusters are already visible in 2-year-old children and are related to maternal characteristics. The findings suggest that obesity prevention should apply an integrated approach to physical activity and dietary intake in early childhood.
\end{abstract}




\section{Introduction}

Several studies have examined the co-occurrence, or 'clustering', of energy balance-related behaviours (EBRBs). Clustering is defined as a combination of behaviours which is more prevalent than expected based on the prevalence of the separate behaviours (Schuit et al., 2002). The potential synergy between EBRBs could be used in obesity prevention interventions, applying an integrated approach in which multiple EBRBs are addressed simultaneously. Furthermore, by identifying behavioural clusters, high risk groups can be identified.

Evidence for the clustering of EBRBs in 7- to 18-year-old children and adolescents is extensive. However, little is known about EBRB clustering in children younger than 7, though attention to this age group is increasing. DuRant and colleagues observed 3- and 4-year-old children and found an inverse correlation between television viewing and time spent being physically active (DuRant et al., 1994). Dietary clusters were found in 2- to 3-year-old children from low-income families, as well as in 4-year-olds, reflecting behavioural combinations that contribute either positively or negatively to the energy balance (Knol et al., 2005; Northstone \& Emmett, 2005). Only one study has examined clustering in young children across dietary intake and physical activity (Lioret et al., 2008). This study reported two EBRB clusters in 3- to 6-year-olds: 1 characterized by high levels of physical activity and varied food intake, and 1 by sedentary and snacking behaviour. However, as yet it is unclear whether these clusters already exist in even younger children.

The aim of this study was to examine EBRB clustering in 2-year-old children. We hypothesized that EBRBs contributing to a positive energy balance are already clustered at the age of 2 years, both within and across behavioural domains.

\section{Methods}

The Child, Parent and Health: Lifestyle and Genetic Constitution (KOALA) Birth Cohort Study, The Netherlands, is a prospective cohort study which started in 2000. Pregnant women were partially recruited from an existing cohort on pregnancyrelated pelvic girdle pain, and partially through alternative channels (Kummeling et al., 2005). All participants signed informed consent, and ethical approval was obtained from the Maastricht University/University Hospital Maastricht medical ethics committee. In total, 2834 women completed questionnaires during pregnancy and regularly after birth. Questionnaires regarding 2578 2-year-old children were returned $(91.0 \%)$. In all, $51.2 \%$ of the children were male, and more than half $(58.5 \%)$ attended daycare. The age of the mother during pregnancy ranged from 17 to 45 years (mean: 32 ), and the country of birth was in most cases the Netherlands (96.5\%). A total of $48.7 \%$ of the mothers had a high education level, $37.6 \%$ a medium education level and $13.7 \%$ a low education level. A total of $17.9 \%$ of the 
mothers were recruited through alternative channels and could have alternative lifestyles with regard to dietary habits (e.g., vegetarian, organic), child rearing, vaccination schemes, or antibiotics use.

When the children reached the age of 2 years, the parents (mostly mothers) completed a questionnaire regarding their child's EBRBs during the preceding month (i.e., including both weekdays and weekend-days). The following activity behaviours were assessed: television (TV) viewing, computer use and playing outside. TV viewing was measured by asking 'How much time does your child spend watching television/videos?' The response categories were 'My child does not watch television/videos', 'Up to 15 minutes per day', '15-60 minutes per day', '1-2 hours per day' and 'More than 2 hours per day'. Computer use was assessed with a similar item, while playing outside was measured using two questions. The first was 'Does your child play outside? (Yes/No) If yes, how often?' (Daily/once every two days/1-2 times per week/rarely). The second was 'How long does your child play outside on those occasions?' (Less than 1 hour at a time/1-2 hours at a time/more than 2 hours at a time).

Dietary intake was assessed by asking parents how often their child consumed the following 13 food items: brown bread (including both whole-wheat bread and brown bread), white bread, raw vegetables, cooked vegetables, fresh fruit, squash concentrate, soft drinks with little or no sugar (light), other soft drinks, chocolate milk, chocolate, cookies and pastry, chips and other salty snacks, and sweets. Response categories were 'never', 'less than once per week', '1-3 times per week', '4-6 times per week', 'once per day', 'twice per day', ' 3 times per day' and 'more than 3 times per day'. Portion size was not assessed. Cooked and raw vegetables were combined into one variable representing total vegetable intake frequency. Cookies, chocolate, chips and sweets were aggregated to represent total snack intake frequency. Finally, squash concentrate, sugar-sweetened soft drinks and chocolate milk were combined to represent total sugar-sweetened beverage intake. This category was solely based on sugar content of the drinks.

All activity behaviour items were re-coded into average minutes per day, while the food variables were recoded into average daily intake frequency. For answer categories that comprised a range of response possibilities, the midpoint value of the category was used for further calculation. For example, the category of watching 1 to 2 hours of TV a day was translated into 90 minutes a day and multiplied by the frequency of the behaviour in order to calculate the total number of minutes per week, which was then divided by seven to calculate the average per day.

Parents were asked to report the weight and height of the child as assessed at his/her periodic screening at an infant welfare centre at age 2 . Weight and height were used to calculate the child's Body Mass Index (BMI) - i.e., weight $/(\text { height })^{2}$ $\left(\mathrm{m} / \mathrm{kg}^{2}\right)$. Because of variations in measurement dates at the infant welfare centres, BMI was only calculated for children that visited these centres between the age of 
22 and 26 months old (i.e., 24 months $+/-2$ months). Because of this restriction, a valid BMI score could not be computed for $7.2 \%$ of the children. To categorize children as non-overweight (i.e., normal or underweight), overweight or obese, the BMI cut-off points defined by Cole et al. were applied (Cole, Bellizzi, Flegal, \& Dietz, 2000). Mothers and fathers were also asked to indicate separately whether they perceived themselves to have severe overweight (yes/no). Additionally, the questionnaire asked about mother's country of birth, age and highest completed education, season of questionnaire completion and alternative lifestyle (yes/no). The highest education completed by the mother was categorized into 3 levels (low, medium and high), in line with international classification systems (Eurostat, 2007). Low education level comprised elementary school, lower vocational education and lower general secondary education. Medium educational level comprised intermediate vocational education, higher general secondary education and collegeprep. Higher education level comprised higher vocational education and college.

All analyses were performed using SPSS software version 13.0 (Chicago, Illinois). Cases with missing values were excluded per analysis. Independent $t$ tests were performed to test sex and weight status differences. Bivariate correlations between intake frequencies and activity durations were calculated using Spearman's rank correlations. Interpretation of the correlations was based on Cohen's guidelines (Cohen, 1988): absolute correlations (r's) between .1 and .3 were considered small; $.3-.5$ medium; and $\geq .5$ large. Principal component analysis with oblique rotation was performed to examine behavioural clustering. All variables were standardized, and a scree plot was used to determine the number of components. Items with absolute component loadings larger than .3 were considered part of the component, in line with previous research (Field, 2005), although cut-offs in other studies were found to vary between .2 and .6. Cluster scores were computed for each child as the sum of all behavioural item scores multiplied by their corresponding component loadings (Lioret et al., 2008). These cluster scores were then used as dependent variables in backward linear regression analyses to examine the relationship between background characteristics and cluster scores. The independent variables were sex and weight status of the child, parental obesity and alternative lifestyle, maternal education, age and country of birth, and season of questionnaire completion. In all analyses, $\mathrm{p}$-values $<.05$ were considered statistically significant.

\section{Results}

Of the 2392 children with complete weight and height data, 207 (8.7\%) were overweight, and $36(1.5 \%)$ were obese. A total of $5.2 \%$ of the mothers and $2.8 \%$ of the fathers reported being obese. Furthermore, $2.5 \%$ of the children of obese mothers were obese themselves, as compared to $1.4 \%$ of the children with a nonobese mother. For the fathers, $1.6 \%$ of the obese and $1.4 \%$ of the non-obese fathers had an obese child. 
On average, the children played outside for approximately 1 hour per day (see Table 3.1). Boys played outside significantly longer than girls $(p<.05), 69$ minutes per day $(\mathrm{SD}=39)$ for the boys compared with 66 minutes $(\mathrm{SD}=40)$ for the girls. Only $4.5 \%$ of the children used computers. The average computer time for the children who did use them was 11 minutes per day $(\mathrm{SD}=12)$.

Table 3.1: Average activity behaviour duration of 2-year-old children in the KOALA Birth Cohort Study $\left(\mathrm{N}=2471^{a}\right)$

\begin{tabular}{|c|c|c|c|c|c|}
\hline & $\begin{array}{c}0 \\
\min . / \text { day }(\%)\end{array}$ & $\begin{array}{c}1-14 \\
\text { min./day (\%) }\end{array}$ & $\begin{array}{c}15-59 \\
\text { min./day (\%) }\end{array}$ & $\begin{array}{c}60-119 \\
\text { min./day (\%) }\end{array}$ & $\begin{array}{c}\geq 120 \\
\text { min./day }(\%)\end{array}$ \\
\hline Playing outside & 1.5 & 6.2 & 35.3 & 38.3 & 18.7 \\
\hline TV viewing & 8.1 & 33.8 & 49.1 & 7.9 & 1.1 \\
\hline Computer use & 95.5 & 4.0 & .5 & .0 & .0 \\
\hline
\end{tabular}

${ }^{a} n$ deviates from total sample size because of missing values.

For dietary intake (see Table 3.2), overweight or obese children consumed significantly fewer snacks on average than non-overweight children: $1.5(\mathrm{SD}=1.0)$ versus 1.6 snacks $(\mathrm{SD}=.8)$ per day respectively; $\mathrm{p}<.05$. Light soft drinks were consumed less than once per week on average, only $16.6 \%$ of the children consumed them. With the exception of playing outside and snack intake, there were no significant sex or weight status differences.

Table 3.2: Average dietary intake frequencies of 2-year-old children in the KOALA Birth Cohort Study $\left(\mathrm{N}=2373^{a}\right)$

\begin{tabular}{lcccc}
\hline & $\begin{array}{c}<\mathbf{1} \\
\text { time/ day (\%) }\end{array}$ & $\begin{array}{c}\mathbf{1 - 2} \\
\text { times/ day (\%) }\end{array}$ & $\begin{array}{c}\mathbf{2}-\mathbf{3} \\
\text { times/ day (\%) }\end{array}$ & $\begin{array}{c}\geq \mathbf{3} \\
\text { times/ day (\%) }\end{array}$ \\
\hline Snacks & 26.1 & 44.1 & 20.4 & 9.4 \\
Sugar-sweetened drinks & 70.1 & 13.1 & 10.4 & 6.4 \\
Light soft drinks & 98.3 & 1.0 & .5 & .2 \\
Vegetables & 40.4 & 54.6 & 4.2 & .8 \\
Fresh fruit & 23.9 & 53.1 & 21.3 & 1.7 \\
Brown bread & 10.6 & 20.0 & 66.8 & 2.6 \\
White bread & 94.6 & 3.3 & 2.1 & .0 \\
\hline
\end{tabular}

${ }^{a} n$ deviates from total sample size because of missing values.

The correlations between activity behaviours and food intake behaviours were small (absolute r's between .1 and .3), except for the medium correlation between snacking and sugar-sweetened drink intake $(\mathrm{r}=.37$; see Table 3.3). Behaviours expected to contribute to a positive energy balance (such as television viewing, snacking and sugared drink intake) were positively correlated and inversely related with healthy behaviours, such as playing outside and eating fresh fruit, vegetables and brown bread. 
Table 3.3: Correlations between activity behaviour and dietary intake $\left(\mathrm{N}=2276^{a}\right)$

\begin{tabular}{|c|c|c|c|c|c|c|c|c|c|}
\hline & TV & $\begin{array}{l}\text { Com- } \\
\text { puter }\end{array}$ & Snacks & $\begin{array}{c}\text { Sugar- } \\
\text { sw. } \\
\text { drinks }\end{array}$ & $\begin{array}{c}\text { Light } \\
\text { soft } \\
\text { drinks }\end{array}$ & $\begin{array}{l}\text { Vege- } \\
\text { tables }\end{array}$ & $\begin{array}{l}\text { Fresh } \\
\text { fruit }\end{array}$ & $\begin{array}{c}\text { Brown } \\
\text { bread }\end{array}$ & $\begin{array}{l}\text { White } \\
\text { bread }\end{array}$ \\
\hline \multicolumn{10}{|l|}{ Activity behaviour } \\
\hline Playing outside & -.10 & & & & & .10 & & & \\
\hline TV & - & & .22 & .16 & & -.15 & -.11 & & \\
\hline Computer & & - & & & & & & & \\
\hline \multicolumn{10}{|l|}{ Dietary intake } \\
\hline Snacks & & & - & .37 & .13 & & & & .12 \\
\hline Sugar-sw. drinks & & & & - & .17 & & -.10 & & .16 \\
\hline Light soft drinks & & & & & - & & & & .13 \\
\hline Vegetables & & & & & & - & .28 & & \\
\hline Fresh fruit & & & & & & & - & .19 & -.12 \\
\hline Brown bread & & & & & & & & - & -.23 \\
\hline
\end{tabular}

Principal component analyses revealed 2 clusters (Table 3.4). The first cluster included TV viewing, computer use, eating snacks and drinking sugarsweetened and light drinks ('sedentary-snacking cluster'). The second comprised vegetable, fresh fruit and brown bread consumption, and rare white bread consumption ('fibre cluster'). These 2 clusters were inversely correlated ( $\mathrm{r}=-.14)$, and explained $30.3 \%$ of the variance in the original items.

Table 3.4: Component loadings of principal component analysis on activity behaviour and dietary intake $\left(\mathrm{N}=2097^{a}\right)$

\begin{tabular}{lcc}
\hline & \multicolumn{2}{c}{ Cluster } \\
\cline { 2 - 3 } & 1 & 2 \\
\hline Activity behaviour & -.015 & .151 \\
$\quad$ Playing outside & .479 & -.135 \\
Television & .326 & .122 \\
Computer & & \\
\hline Dietary intake & .733 & -.027 \\
$\quad$ Snacks & .669 & -.009 \\
Sugar-sweetened drinks & .361 & -.097 \\
Light soft drinks & -.106 & .468 \\
$\quad$ Vegetables & -.094 & .532 \\
$\quad$ Fresh fruit & .299 & .736 \\
$\quad$ Brown bread & .017 & -.606 \\
$\quad$ White bread & & \\
\hline a deviates from total sample size due to missing values. \\
Results of oblique principal component analysis.
\end{tabular}


Regression analyses on the cluster scores showed that higher sedentarysnacking cluster scores were predicted by maternal obesity (standardized regression coefficient $\beta=.06 ; \mathrm{p}<.01)$, and by low and medium educational level $(\beta=.13 ; \mathrm{p}<.001$ and $\beta=.16 ; \mathrm{p}<.001$ respectively, compared with high educational level). With regard to the fibre cluster, low $(\beta=-.17 ; \mathrm{p}<.001)$ and medium $(\beta=-.15 ; \mathrm{p}<.001)$ maternal educational level compared with high educational level were significantly inversely related to cluster score. Reported regression coefficients were corrected for potential confounding influences of alternative lifestyle and season of questionnaire completion. Sex, child and father's weight status, and mother's country of birth and age were not significantly related to either cluster.

\section{Discussion}

Two interesting behavioural clusters emerged in the present study. The first, a sedentary-snacking cluster, comprised sedentary behaviour and unhealthy dietary intake behaviours such as snacking and soft drink consumption. Children scoring high on this cluster are more likely to develop obesity (WHO/FAO Expert Consultation, 2003). There are several proposed mechanisms to explain the clustering of sedentary behaviours and unhealthy food intake, including the stimulating influence of TV snack commercials (Halford, Boyland, Hughes, Oliveira, \& Dovey, 2007), provision of a context during sedentary activities that promotes passive snacking or overeating (Coon et al., 2001), and the distracting influence of TV watching while eating, which disrupts habituation to food cues (e.g., satiety; Temple, Giacomelli, Kent, Roemmich, \& Epstein, 2007). Second, we identified a fibre cluster characterized by eating fresh fruit, vegetables and brown bread more often and white bread less often. There is convincing evidence for the protective role of dietary fibre against the development of overweight and obesity (WHO/FAO Expert Consultation, 2003).

Light soft drinks are calorie free, and could therefore be expected to cluster with healthy behaviours. Instead, they were associated with sedentary behaviours and unhealthy dietary patterns. This pattern is consistent with earlier findings among adolescents (Striegel-Moore et al., 2006), and may be explained by parents restricting light soft drink consumption because they consider it unhealthy because of potentially negative effects in other health areas.

Parents play a crucial role in the lives of young children. They decide on almost every aspect of the toddler's life, including factors influencing EBRBs and weight gain. Parents control the availability of and exposure to foods, determine daily activity patterns, are role models, practice certain parenting styles, and can provide support and structure for their child (Ebbeling, Pawlak, \& Ludwig, 2002; Golan \& Crow, 2004). When trying to change EBRBs and prevent excessive weight gain in young children, it is therefore necessary to target parents more intensively than is current practice in the obesity prevention field. In this study, maternal 
education was found to significantly influence both cluster scores, with lower educational level being related to EBRBs that could predict obesity risk. This is in line with earlier research (Aranceta, Perez-Rodrigo, Ribas, \& Serra-Majem, 2003; Lioret et al., 2008). Furthermore, maternal obesity was associated with a high sedentary-snack cluster score in the current study. These findings stress the importance of parents in the life of young children, and indicate that interventions should focus on these high risk groups. In contrast to mother's obesity, father's obesity was not related to these 2 clusters, possibly reflecting the traditionally greater role mothers play in rearing young children.

Contrary to expectations, no association was found between the child's weight status and cluster scores. This could be due to the cross-sectional nature of the data; it would therefore be of interest to re-examine the effect of the cluster scores on weight status at a later age. However, it also indicates that at this age, the deleterious effects of unhealthy behaviours have not yet manifested themselves in the child's weight status. This implies a window of opportunity for early prevention. The slightly lower snack intake found in overweight and obese children could be an indication of adaptive behaviour of the parents, with parents already correcting the diet of their children because of emerging overweight of the child. This seems plausible because the child was physically examined (including weight and height measurements) at the infant welfare centre just before filling out the questionnaire.

In interpreting these results, some limitations should be taken into account. Our study population was not a representative sample of the Dutch population. Almost half of the mothers had a high educational level, as opposed to one-third of the 25- to 45-year-old women in the Netherlands (Centraal Bureau voor de Statistiek, 2005). All data, including weight and height, were reported by parents, and could therefore be biased. However, the questionnaires were completed shortly after visits to infant welfare centres, and parents were asked to take down the results of the anthropometric measurements taken there. Overweight and obesity prevalence in the study population were similar to other Dutch prevalence figures (Van den Hurk et al., 2007). However, parental reports of their child's behaviours may have been subject to bias, and results concerning children attending daycare (58.5\%) might be influenced, due to less parental awareness of the child's EBRBs during daycare. Furthermore, the food frequency questions and activity measures were not validated, and not all food intake and activity behaviours were taken into account (e.g., active indoor playing), although the EBRBs examined here are commonly considered the most important risk factors for child and adolescent obesity (Rennie et al., 2005; WHO/FAO Expert Consultation, 2003).

Despite these limitations, this study provides valuable insights and expands the evidence base of EBRB clustering in toddlers. Clustering across dietary intake and activity behaviour has been demonstrated in 2-year-olds. The existence of 
behavioural clusters at this young age calls for an integrated prevention approach in which physical activity and dietary intake are addressed simultaneously. 


\section{Chapter 4: Diet-related restrictive parenting practices. Impact on dietary intake of 2-year- old children and interactions with child characteristics}

This chapter has been published as: Gubbels, J.S., Kremers, S.P.J., Stafleu, A., Dagnelie, P.C., Goldbohm, R.A., de Vries, N.K. \& Thijs, C. (2009). Diet-related restrictive parenting practices. Impact on dietary intake of 2-year-old children and interactions with child characteristics. Appetite, 52, 423-429. 


\begin{abstract}
Aim: This study examined the relationship between diet-related parenting practices, parental characteristics, child characteristics, and 2-year-old child's dietary intake. Methods and Results: Cross-sectional data $(\mathrm{N}=2578)$ originated from the KOALA Birth Cohort Study. Principal component analyses revealed two restrictive parenting practice clusters: a cluster characterized by prohibition of the intake of various snacks and soft drinks, and a separate cluster characterized by prohibition of cookies and cake. Regression analyses showed that these clusters were related to the children's behavioural style (i.e., oppositional, depressive and/or aggressive behaviour) and to educational level, age and alternative lifestyle of the mother. The clusters also had a favourable influence on dietary intake (i.e., restrictive parenting practices were related to less consumption of the restricted (unhealthy) items and higher consumption of items considered to be healthy), which was moderated by child characteristics. The parenting practices showed a stronger association with dietary intake in children with a favourable behavioural style (i.e., non-depressed, low anxious, low overactive), a favourable eating style or a lower BMI.

Conclusions: The findings suggest opportunities for preventive interventions focussing on parents of young children, and indicate that different approaches to parenting practice interventions are needed for different types of children.
\end{abstract}




\section{Introduction}

Childhood overweight and obesity are a growing problem worldwide. In 2005, at least 20 million of the world's children under the age of five were overweight, and it is expected that these numbers will continue to rise (World Health Organization, 2006). Moreover, overweight and obese children often develop into overweight and obese adults (Singh, Mulder, Twisk, van Mechelen, \& Chinapaw, 2008).

One of the main risk factors for overweight in childhood is an unhealthy diet (Rennie et al., 2005). Parents can have a strong influence on their child's health behaviour, including their dietary intake. For instance, parents control the availability of and exposure to food, act as role models, can provide their child with support and structure, and use specific parenting practices (Golan \& Crow, 2004). These parenting practices appear to have a long-lasting impact on dietary habits and food intake, not only during childhood, but throughout life (Puhl \& Schwartz, 2003).

The term parenting practices refers to content-specific acts of parenting, for example food rules (Darling \& Steinberg, 1993). Although several studies have examined the immediate influence of parenting practices on dietary intake and weight in children and adolescents, their results were not consistent. Some studies found favourable associations between diet-related parenting practices and children's dietary intake. Restriction of unhealthy food items was associated with lower consumption of those items (e.g., lower soft drink consumption; De Bruijn, Kremers, de Vries, van Mechelen, \& Brug, 2007), and higher consumption of healthy items (more fruit and vegetable consumption; Zabinski et al., 2006). Such a favourable association was also seen for promotion or imposition of healthy foods, which was associated with lower consumption of unhealthy food items (e.g., lower soft drink consumption, De Bourdeaudhuij \& Van Oost, 2000; less dietary fat intake, Zabinski et al., 2006) and higher consumption of healthy items (more fruit and vegetable consumption, Zabinski et al., 2006). Furthermore, high parental control was associated with higher intake of healthy snacks (Brown \& Ogden, 2004). Other studies, however, found restriction to be paradoxically associated with increased intake and preference for the restricted foods, and with overeating and weight gain (e.g., Fisher \& Birch, 1999; Liem, Mars, \& De Graaf, 2004; Montgomery, Jackson, Kelly, \& Reilly, 2006). Promotion of healthy foods or pressure to eat such foods (e.g., vegetables) was associated with lower preference for and intake of these foods (Matheson, Robinson, Varady, \& Killen, 2006). Moreover, higher control over food intake in general was associated with higher unhealthy snack intake (Brown \& Ogden, 2004). This paradoxical effect is assumed to arise because parental control impedes the development of self-control over food intake (Tiggemann \& Lowes, 2002), a mechanism which was confirmed by the findings of Johnson and Birch (1994), who reported reduced ability to compensate 
food intake in children of parents who exerted high levels of control (Johnson \& Birch, 1994).

Several other findings complicate matters even more, but could also provide the key to explaining these conflicting findings. Van der Horst and colleagues (2007) reported that the influence of parenting practices depended on the parents' more general parenting style, with practices having the desired influence on child behaviour only when parents were moderately strict and highly involved (Van der Horst et al., 2007). This is in line with the theoretical model proposed by Darling and Steinberg (1993), who argue that general parenting styles provide the context in which specific parenting practices operate. In line with this model, the influence of parenting practices has also been found to be moderated by the child's personality traits (De Bruijn et al., 2007) and gender (e.g., Fisher \& Birch, 1999; Montgomery et al., 2006; Zabinski et al., 2006).

The influence of parental and child characteristics on parenting practices has been examined in several studies. While efforts to identify which parents use what practices can help to identify target groups for interventions, an examination of the interactions of parent and child characteristics with parenting practices may also help explain the above moderation effects. With regard to parental factors, stricter parenting practices have been found to be associated with higher parental level of education (e.g., Brown, Ogden, Vogele, \& Gibson, 2008) and socioeconomic status (Hupkens, Knibbe, Van Otterloo, \& Drop, 1998), as well as with both lower (e.g., Brown et al., 2008; Liem et al., 2004; Montgomery et al., 2006) and higher (Keller, Pietrobelli, Johnson, \& Faith, 2006) parental BMI, with dietary restraint by the parents (Birch \& Fisher, 2000; Fisher \& Birch, 1999; Tiggemann \& Lowes, 2002), with parents who are older (Brown et al., 2008; Keller et al., 2006; Liem et al., 2004) and with parents who stay at home to take care of the children (Brown et al., 2008). Furthermore, the children's age (e.g., De Bruijn et al., 2007), gender (Stang, Rehorst, \& Golicic, 2004) and weight (both measured and perceived; e.g., Birch \& Fisher, 2000; Keller et al., 2006; Matheson et al., 2006; Tiggemann \& Lowes, 2002) were associated with parenting practices.

It is often not clear whether a child's dietary intake and weight are causes or consequences of certain parenting practices. In practice, however, both are probably true (Faith \& Kerns, 2005). Birch and Fisher (2000) have therefore proposed and validated a cyclical model, in which the relative weight of the child is a predictor of restrictive parenting practices, as it influences the parents' perceptions of their child's overweight risk, but in which the child's weight is also a consequence of restrictive parenting practices, through the influence of these practices on energy intake regulation. Overweight children and their parents therefore find themselves in a vicious circle, in which child overweight leads to stricter practices, which in turn may lead to even more weight gain (Birch \& Fisher, 2000). 
A specific dietary behaviour that puts a child at risk for becoming overweight is a high consumption of energy-dense foods such as snacks and sugarsweetened beverages (Rennie et al., 2005). It is worrying that such eating habits find their origin in early childhood (Savage et al., 2007), and track into later life (Kelder et al., 1994). It is therefore crucial to study parental influences even in very young children. Note that it is not single behavioural acts, but combinations of multiple risk behaviours and the complex interplay between them that are expected to lead to excess weight gain (Rennie et al., 2005). 'Obesogenic' behaviours have repeatedly been shown to cluster (i.e., co-occur) at various ages (e.g., Kremers, van der Horst, \& Brug, 2007; Lioret et al., 2008; Utter et al., 2003). Nevertheless, previous studies have not used a clustering approach to examine diet-related parenting practices. Clusters of parenting practices could be indicators of a wider obesogenic parental context, and insights into the clustering of parenting practices could help inform the development of interventions aimed at improving diet-related parenting.

The present study examined whether diet-related parenting practices cluster in parents of 2-year-old children, and how these clusters are related to the child's dietary intake. We also examined associations of parental and child characteristics with the practice clusters, and whether these child characteristics influenced the impact of the parenting practices on the child's dietary intake. We hypothesized that restrictive parenting practices would indeed cluster, and that these restrictive clusters would be associated with less unhealthy dietary behaviour on the part of the children. We further hypothesized that the practices would be related to distinct parent and child characteristics, and that the impact of the practices was moderated by child characteristics (see Figure 4.1).

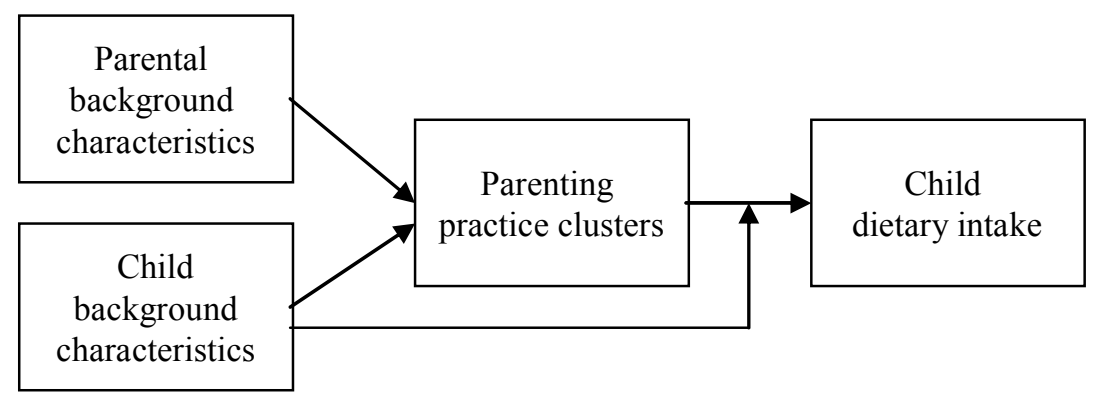

Figure 4.1: Hypothesized model of the interaction between parent and child with respect to dietary intake, restrictive practice clusters and background characteristics 


\section{Methods}

\section{Respondents and procedure}

The KOALA Birth Cohort Study (The Netherlands) is a prospective cohort study which started in the year 2000. KOALA is the Dutch acronym for Child, Parent and health: Lifestyle and Genetic constitution. Pregnant women were recruited from an existing cohort for a study of pregnancy-related pelvic girdle pain, as well as through recruitment channels among 'alternative lifestyle' circles (e.g., anthroposophist midwives and general practitioners, Steiner schools and organic food shops; Kummeling et al., 2005). The latter group of women (17.9\%) could have an alternative lifestyle in terms of dietary habits (e.g., preferring organic foods), child rearing, vaccination schemes or antibiotics use. All participants signed informed consent, and approval was obtained from the Maastricht University/University Hospital Maastricht medical ethics committee. In total, 2834 women participated and completed questionnaires during pregnancy, and when their child was 3, 7, 12 and 24 months old. At the time the children were 2 years old, 2578 questionnaires had been returned (91.0\%). In all, 51.2\% of these children were male. The mean age of the mothers during pregnancy was 32, ranging from 17 to 45 years. The country of birth of the mother was the Netherlands in $96.5 \%$ of the cases. A total of $48.7 \%$ of the mothers were highly educated, while $37.6 \%$ had a medium educational level and $13.7 \%$ had a low educational level.

\section{Questionnaire}

When the children were 2 years old, parents completed a questionnaire regarding their diet-related restrictive parenting practices (i.e., restrictive rules on food intake) and their child's dietary intake during the month preceding the questionnaire. Several other child and parent characteristics were also assessed.

\section{Diet-related restrictive parenting practice clusters}

Parenting practices were assessed by asking parents: 'Are there specific foods that you do not allow your child to eat or drink?' (no/yes). Parents who answered 'yes' were then asked to indicate which of the following foods the child was not allowed to eat: 'Sweets', 'Cookies', 'Cake', 'Soft drinks', 'Crisps' and 'Sugar'. Principal component analysis with oblique rotation was used to form clusters of practices. Both a scree plot and the Eigen-values were used to determine the number of components (Field, 2005). For interpretation purposes, an absolute cut-off of .5 was used with regard to component loadings. Cluster scores were computed for each parent-child combination as the sum of the component loadings corresponding to the practices that were applied by the parent. 


\section{Dietary intake}

Dietary intake was measured by asking parents how often their child consumed 65 food items. Six items corresponding with the restrictive parenting practices were selected for analyses: 'Sweets', 'Chocolate', 'Cookies and Cake', 'Soft drinks with little or no sugar (light)', 'Other soft drinks' and 'Crisps and other savoury snacks'. These six items were complemented with three items on vegetable and fruit intake: 'Raw vegetables', 'Cooked vegetables' and 'Fresh fruit'. Response options were 'Never', 'Less than once a week', '1-3 times a week', '4-6 times a week', 'Once a day', 'Twice a day', 'Three times a day' and 'More than three times a day'. Cooked and raw vegetables were combined into one variable measuring total vegetable intake frequency. The food items were recoded into weekly intake frequency.

\section{Child characteristics}

Several child background characteristics were assessed. The children's behavioural style was identified using a selection of questions from a translated version of the Child Behaviour Checklist (Koot, Van Den Oord, Verhulst, \& Boomsma, 1997). A total of 51 items were used to assess whether various behavioural characteristics applied to the child. Response options were 'Not applicable at all' (0), 'A little / sometimes' (1), and 'Clearly / often' (2). The items were divided into 5 subscales measuring oppositional, depressed, aggressive, anxious and overactive behaviour. These scales were derived from factor analyses, and validated in a Dutch sample (Koot et al., 1997). An example of an item measuring oppositional behaviour is 'The child's demands must be met immediately'. An example of an item measuring depressed behaviour is 'The child looks unhappy without good reason'. An example of an aggressive behaviour item is 'The child physically attacks people'. An example of anxious behaviour is 'The child is afraid to try new things'. An example of an item measuring overactive behaviour is 'The child cannot sit still, is restless or hyperactive'.

Eating style at the age of one year was assessed by means of the question: 'Can you describe the eating behaviour of your child'. Response options were 'Eager', 'With reluctance', 'With pleasure', 'Fast, 'Slow', 'Doesn't like many things', 'Likes many things'. Parents could indicate for each response option whether or not it applied to their child (yes/no).

Parents were asked to report the height and weight of their child as periodically assessed at infant welfare centres at age 1 and 2, under the Dutch preventive health system. Weight and height were used to calculate Body Mass Index (BMI, i.e., weight / (height $)^{2} ; \mathrm{kg} / \mathrm{m}^{2}$ ). In accordance with international standards for 2-year-olds, boys with a BMI above 18.41 and girls with a BMI above 18.02 were considered to be overweight. The cut-offs for obesity are 20.09 for boys and 19.81 for girls (Cole et al., 2000). Because these cut-offs were not defined for one-year-olds, BMI at age one was categorized into high and low, with a cut-off at 
the median $\left(17.01 \mathrm{~kg} / \mathrm{m}^{2}\right)$. We also recorded the child's gender, as well as the number of hours the child spent at day care.

\section{Parental characteristics}

Parents were asked to indicate their own weight status, i.e., whether one or both parents were obese (yes/no). The number of hours a week that both parents were at work was also assessed. Additionally, country of birth, age and highest completed education of the mother were assessed. Education of the mother was categorized into three levels (low, medium and high), in line with international classification systems (Eurostat, 2007; United Nations Educational Scientific and Cultural Organization, 1997). Finally, we recorded whether the parents had been recruited through conventional or 'alternative' channels.

\section{Data analyses}

The parenting practice cluster scores were used as dependent variables in backward linear regression analyses, to examine the relation with parental and child characteristics. Linear regression was used to assess the association between cluster scores and the reported consumption frequency of sweets, chocolate, cookies and cake, sugar-sweetened and light soft drinks, crisps, vegetables and fruit. These analyses were corrected for the child and parent background characteristics mentioned above. Finally, interactions between child characteristics and the impact of the practice clusters on the child's dietary intake were explored by examining the explanatory value of adding interaction terms to the regression as described above. In all analyses, $\mathrm{p}$-values $<.05$ were considered statistically significant.

\section{Results}

Mean BMI of the children at age 2 was $16.4 \mathrm{~kg} / \mathrm{m}^{2}$. No valid BMI score could be computed for $7.2 \%$ of the children because of missing data on weight and height. A total of $207(8.7 \%)$ of the remaining children were overweight, and $36(1.5 \%)$ were obese. Further, $5.2 \%$ of the mothers and $2.8 \%$ of the fathers reported to be obese. Mean number of hours at work per week was 19 hours (SD =12) for the mothers and 40 hours $(\mathrm{SD}=10)$ for the fathers. Children spent an average of 11 hours per week $(\mathrm{SD}=11)$ at day care.

Table 4.1 shows the percentage of parents who applied the various food restrictions. In total, almost half of the parents had certain restrictive parenting practices with regard to one or more of these food items $(\mathrm{N}=1149,44.6 \%)$. Frequencies for most restrictions were, however, rather low. The most common restriction concerned soft drink consumption (applied by $42.2 \%$ of the parents), while least common was restriction of cookies ( $1.4 \%$ of the parents). 
Table 4.1: Frequencies of diet-related restrictive parenting practices in 2-year-old children of the KOALA Birth Cohort Study $(\mathrm{N}=2578)$

\begin{tabular}{lc}
\hline Child is not allowed to consume: & N (\%) \\
\hline Sweets & $255(9.9)$ \\
Cookies & $37(1.4)$ \\
Cake & $106(4.1)$ \\
Crisps & $153(5.9)$ \\
Sugar & $223(8.7)$ \\
Soft drinks & $1089(42.2)$
\end{tabular}

Parenting practices were assessed by asking parents to indicate which of the six items their child was not allowed to eat.

Two clusters of diet-related restrictive parenting practices were found (Table 4.2). Cluster 1 included rules regarding the intake of sweets, crisps, sugar and soft drinks. Cluster 2 included the absence of rules on the consumption of cookies and cake. The component loadings all had absolute loadings $>.5$.

Table 4.2: Component loadings of Principal Component Analysis on diet-related restrictive parenting practices $(\mathrm{N}=2578)$

\begin{tabular}{lcc}
\hline & \multicolumn{2}{c}{ Cluster } \\
\cline { 2 - 3 } & 1 & 2 \\
\hline No sweets & .67 & -.24 \\
No cookies & -.03 & -.88 \\
No cake & .06 & -.81 \\
No crisps & .67 & -.03 \\
No sugar & .53 & -.31 \\
No soft drinks & .81 & .22 \\
\hline
\end{tabular}

The two clusters were inversely correlated $(\mathrm{r}=-.33)$, and the total variance explained by the clusters was $59.6 \%$. For interpretation purposes, scores on the second cluster were multiplied by -1 (so that a higher score means more restriction), which is statistically justified, since the scale for measuring the parenting practices is dichotomous. This means that clustering of the absence of rules, also indicates clustering of the presence of rules.

Table 4.3 shows the associations of child and parent background characteristics with the parenting practices cluster scores. Higher scores on the first cluster were associated with oppositional behaviour of the child and high educational level and alternative lifestyle of the parents. Lower scores were related to depressed and aggressive behaviour of the child. High scores on the second cluster, i.e., more restriction regarding cookie and cake intake, were also inversely related to aggressive behaviour. Higher scores on cluster 2 (more restriction regarding cookies and cake) were also positively related to oppositional behaviour of the child, alternative lifestyle and older age of the mother. The total number of rules was higher for oppositional children and lower for aggressive children. 
Parents with an alternative lifestyle and/or a high educational level also reported more rules.

Table 4.3: Associations of background characteristics with diet-related parenting practice cluster scores and total number of diet-related restrictive parenting practices (Standardized regression coefficients backward regression, $\mathrm{N}=1958^{a}$ )

\begin{tabular}{lccc}
\hline & $\begin{array}{c}\text { Cluster 1: Restrictive } \\
\text { practices regarding } \\
\text { snacking }\end{array}$ & $\begin{array}{c}\text { Cluster 2: Restrictive } \\
\text { practices regarding } \\
\text { cookies and cake }\end{array}$ & Total number of rules \\
\hline $\begin{array}{l}\text { Oppositional behaviour } \\
\text { of child }\end{array}$ & $.08^{* *}$ & $.07^{*}$ & $.08^{* *}$ \\
$\begin{array}{l}\text { Depressive behaviour of } \\
\text { child } \\
\text { Aggressive behaviour of } \\
\text { child }\end{array}$ & $-.05^{*}$ & - & - \\
$\begin{array}{l}\text { Education level of mother } \\
\text { (high } v \text { s. low/medium) }\end{array}$ & $-.05^{*}$ & $-.08^{* *}$ & $-.07^{*}$ \\
$\begin{array}{l}\text { Alternative lifestyle } \\
\text { (Yes } v s . \text { No) }\end{array}$ & $.06^{* *}$ & - & $.06^{*}$ \\
\begin{tabular}{l} 
Age of the mother \\
\hline $\begin{array}{l}\text { Total variance explained } \\
\text { by the model }\left(\mathrm{R}^{2}\right)\end{array}$
\end{tabular} & $.23^{* *}$ & $.14^{* *}$ & $.23^{* *}$ \\
\hline
\end{tabular}

$* p \leq .05 ; * * p \leq .01$.

${ }^{a} N$ deviates from total sample due to missing values.

Scores on the second cluster were multiplied by -1 so that a higher score means more restriction. Excluded (non-significant) variables for all three scores: Gender, BMI at 1 and 2 years, anxious or overactive behaviour, eating style, obesity of mother and obesity of father, hours at work a week for father and mother, hours a week at day care, and country of birth of the mother.

The association between the cluster scores and the intake of specific foods was examined using linear regression (see Table 4.4). Both clusters were significantly inversely related to unhealthy intake behaviours (i.e., consumption of sweets, chocolate and cookies and cake), and positively associated with fruit and vegetable intake. Higher scores on cluster 1 were further associated with lower intake of both sugar-sweetened and light soft drinks, and with lower crisps intake. Cluster 2 was positively related to soft drink consumption. 
Table 4.4: Association between diet-related parenting practice cluster scores and dietary intake (Standardized regression coefficients, $\mathrm{N}=1931^{a}$ )

\begin{tabular}{|c|c|c|c|c|c|c|c|c|}
\hline & Sweets & Chocolate & $\begin{array}{l}\text { Cookies } \\
\text { and cake }\end{array}$ & $\begin{array}{l}\text { Sugar- } \\
\text { sw. soft } \\
\text { drinks }\end{array}$ & $\begin{array}{l}\text { Light soft } \\
\text { drinks }\end{array}$ & Crisps & $\begin{array}{l}\text { ege- } \\
\text { ables }\end{array}$ & Fruit \\
\hline Cluster 1 & $-.19 \mathrm{~m}$ & -.10 & & $-.16^{* *}$ & .14 & $-.25^{\circ}$ & & \\
\hline Cluster 2 & $-.08 * *$ & $-.05 *$ & & $.06^{* *}$ & & .01 & & \\
\hline \multicolumn{9}{|c|}{$\begin{array}{l}* p \leq .05 ; * * p \leq .01 . \\
\text { a } N \text { deviates from total sample due to missing values. } \\
\text { Scores on the second cluster were multiplied by }-1 \text { so that a higher score means more restriction. } \\
\text { Cluster } 1 \text { : Restrictive practices regarding snacking; Cluster 2: Restrictive practices regarding cookies } \\
\text { and cake. Reported } \beta \text {-weights are corrected for confounding influence of background characteristics } \\
\text { (child's behaviour, eating style, number of hours at day care, BMI at age } 1 \text { and 2; mothers' age, } \\
\text { education level, number of hours at work, country of birth; fathers' number of hours at work; parents ' } \\
\text { obesity and alternative lifestyle). Sugar-sw. = sugar-sweetened. }\end{array}$} \\
\hline
\end{tabular}

We found several significant interactions between child characteristics and the impact of parenting practice clusters on dietary intake. All results indicate that the parenting practices had a stronger favourable impact (i.e., led to less consumption of unhealthy foods and more intake of healthy items) on children with more 'favourable' characteristics (Figure 4.2). With regard to the children's behavioural style, the first cluster only had an impact $(p<.01)$ on cookie and cake intake in non-depressed children (depressed score $=0, \mathrm{~N}=1412$; see Figure 4.2a). Depressed behaviour of the child further weakened the relationship between cluster 1 (snacking restriction) and crisps intake $(\mathrm{p}<.05$; see Figure $4.2 \mathrm{~b})$. High anxious behaviour (above median) nullified the positive influence of cluster 1 on vegetable intake ( $<.05$; Figure 4.2c). Furthermore, the unfavourable influence of cluster 2 on sugar-sweetened soft drink intake was only found in overactive children $(p<.05$; Figure $4.2 \mathrm{~d}$ ). An eating style in which the child liked many foods strengthened the influence of cluster 1 (restricting snacks) on sweets intake ( $<.05$; Figure $4.2 \mathrm{e})$. The influence of the first cluster on chocolate intake was weaker for children who ate with reluctance $(p<.05$; see Figure $4.2 \mathrm{f})$, and was only present in children who were not reported to be slow eaters $(\mathrm{p}<.05$; Figure $4.2 \mathrm{~g})$. As regards vegetable intake, cluster 1 had a positive influence for children who were not reported to be slow eaters ( $<<.05$; Figure $4.2 \mathrm{~h}$ ). With regard to weight status, BMI at age 1 interacted with the second cluster $(p<.01)$, in such a way that the positive influence of restrictive parenting on cookie and cake intake was only found for children with a below-median BMI $(\mathrm{BMI}=17.01$; Figure $4.2 \mathrm{i})$. BMI at age 2 moderated the influence of cluster 1 on both sugar-sweetened soft drink and crisps intake $(\mathrm{p}<.05)$, with a weaker impact in overweight or obese children (see Figure $4.2 \mathrm{j}$ and $4.2 \mathrm{k}$ ). 


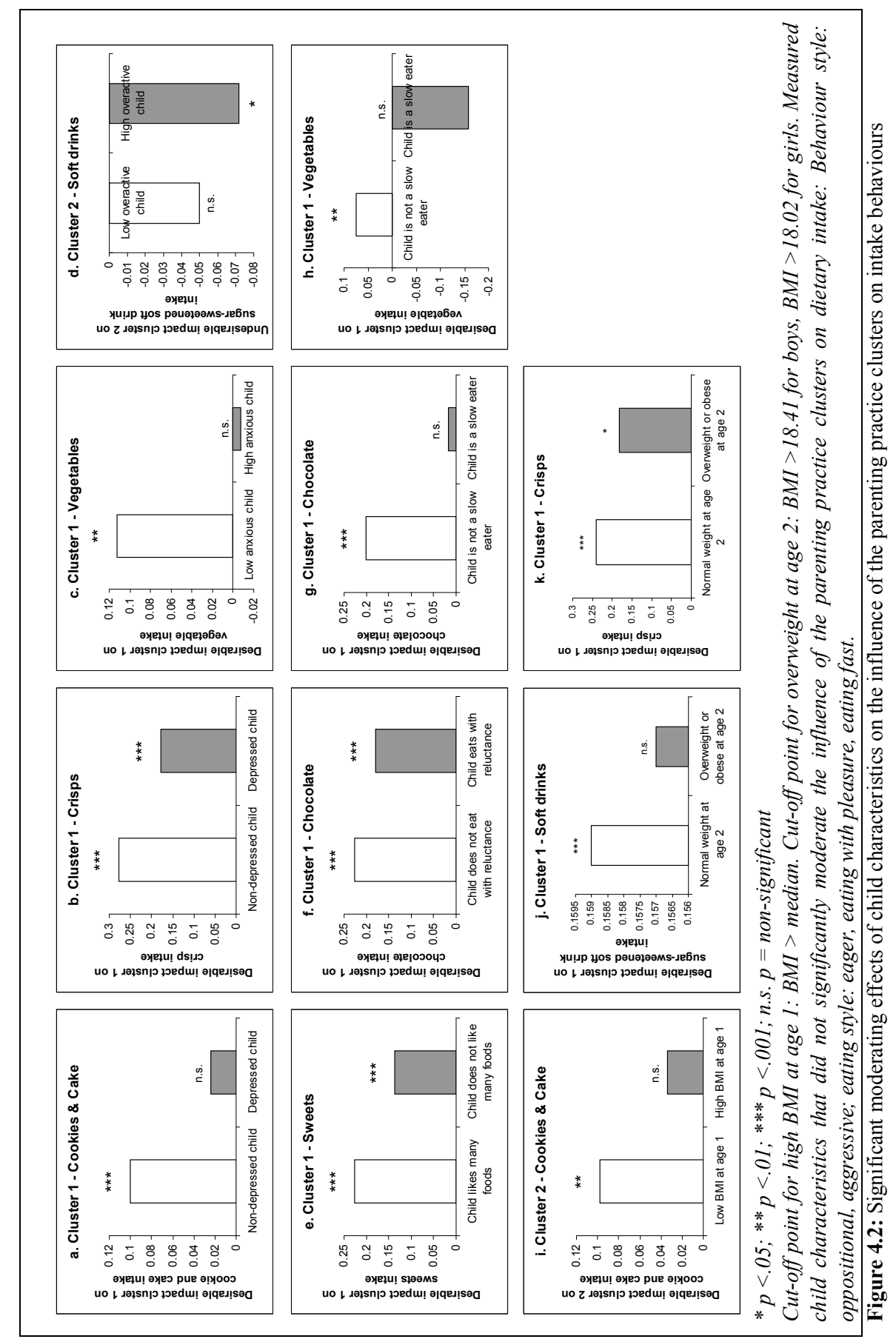




\section{Discussion}

The current study demonstrated the existence of clusters of diet-related restrictive parenting practices. The first cluster was characterized by prohibition of consumption of sugar, snacks such as sweets and crisps, and soft drinks. A second cluster was characterised by prohibition of cookies and cake.

From the lifestyle point of view, it is obvious why practices prohibiting the intake of unhealthy food cluster together: rules are part of parents' views on child rearing and lifestyle, and may thus reflect a more general parenting style. A parenting style is the constellation of attitudes held towards and communicated to the child that create an emotional climate in which the parents' behaviours are expressed (Darling \& Steinberg, 1993). This general climate probably influences which parenting practices are applied (Kremers, Brug, de Vries, \& Engels, 2003). It is not clear why a separate cluster was found for restrictions regarding cookies and cake. The fact that cookies and cake are consumed at different moments and occasions than the items in the first cluster could play a role.

The parenting practice clusters seemed primarily dependent on parental lifestyle, as those parents with an alternative lifestyle were stricter with respect to diet than traditional parents. This probably reflects the parents' own dietary habits, which they may also want their child to practise. Some of these alternative lifestyle parents $(24.7 \%)$ practiced a vegetarian, organic or other type of alternative dietary style, which are characterized by relatively healthy eating habits and which are associated with a lower risk of various chronic diseases (American Dietetic Association and Dieticians of Canada, 2003). High educational level of the mother was significantly related to more restrictive parenting practices with respect to snack intake (cluster 1), and a higher total number of rules. Other studies also found a positive relationship between educational level and strictness (Brown et al., 2008). Furthermore, older mothers imposed more restrictions regarding cookies and cake, which is also in agreement with previous research (Brown et al., 2008; Keller et al., 2006; Liem et al., 2004). The current study found no association between parental obesity and the parenting practice clusters.

The children's behavioural style was significantly related to the degree of strictness of the parents: the more oppositional (e.g., stubborn, demanding, jealous) the child, the higher the scores on the practice clusters, and the more rules were imposed. A potential explanation for this finding is that parents may feel that these children need more guidance and rules. An alternative explanation might be that these children became oppositional because of the restrictions their parents imposed on them. A familial component might also be involved: the parents of these oppositional children might be oppositional themselves, which may be reflected in the food rules they apply, especially when food rules diverge from conventional rules. Furthermore, children's depressed and aggressive behaviours were associated with less dietary restrictions. Parents may be less strict to a depressed child and may 
impose fewer rules to avoid their child engaging in aggressive behaviour. Few studies have examined the influence of child behaviour in general on diet-related parenting practices. De Bruijn and colleagues (2007) found a significant moderating effect of a personality factor referred to as 'agreeableness' (i.e., a tendency to be pleasant and accommodating in social situations) on the influence of parenting practices on soft drink consumption, but they did not examine whether agreeableness was associated with the use of certain parenting practices. In addition, several studies reported an association between specific eating behaviours, such as inhibited eating and eating in the absence of hunger, and parenting practices (see for instance the review of Faith \& Kerns, 2005), but as Birch and Fisher (2000) elucidated, this influence is likely to be bi-directional. The current study did not find a significant relation between the children's general eating styles and BMI on the one hand and the parenting practice clusters on the other.

In line with several other studies (De Bourdeaudhuij \& Van Oost, 2000; De Bruijn et al., 2007; Zabinski et al., 2006), the current study found that diet-related parenting practices did have the intended effect on food intake. However, the previous studies showing these results all involved adolescents. To our knowledge, the current study is the first to find a favourable influence of restriction on the intake of energy-dense foods and snacks in young children. Thus, we failed to confirm the paradoxical effect of increased consumption of restricted foods reported in several studies (e.g., Brown \& Ogden, 2004; Fisher \& Birch, 1999; Matheson et al., 2006; Montgomery et al., 2006), including studies among toddlers. However, these studies generally involved intake in an unrestricted setting, with free access to food. The current study measured mean intake in normal life, a setting that is seldom unrestricted for 2-year-olds.

The practice clusters not only influenced the intake behaviours they specifically related to, but were also associated with other dietary behaviours: higher scores on the clusters were associated with higher fruit and vegetable intake. Similar results were found in a study among adolescents, in which restricting unhealthy foods was associated with higher fruit and vegetable intake (Zabinski et al., 2006). This wide-ranging influence of parenting practices could possibly be due to clustering of intake behaviours (e.g., Lioret et al., 2008). An alternative explanation may be that other parenting practices, which we did not measure, play a role.

The influence of both parenting practice clusters on intake was moderated by several child characteristics. Restrictive parenting practices were found to have greater impact in children with 'favourable' characteristics, and smaller impact on children with higher depressive, anxious or overactive behavioural scores. In addition, higher BMI at both age 1 and 2 was associated with a weaker influence of the parenting practices on intake. A final child characteristic that was found to moderate the influence of parenting practices on dietary intake was the child's eating style. The influence of parenting practices proved stronger in children who 
liked many foods, whereas the influence was weaker for children who were slow or reluctant eaters. Although our study did not confirm the paradoxical adverse effects of parenting practices found in some studies, it provides some indication why parenting practices do not always result in favourable effects. In contrast to what was found in earlier studies (Fisher \& Birch, 1999; Montgomery et al., 2006; Zabinski et al., 2006), the current study did not find that gender moderated the influence of parenting practices on intake.

A strength of the current study is the large number of participants $(\mathrm{N}=$ 2578). Previous research on the effect of parenting practices on intake mostly included no more than 200 participants, and none included more than 900 participants. However, caution is warranted when generalizing the results to the larger population, as the current sample has an overrepresentation of highly educated parents and parents with an alternative lifestyle, as well as relatively low overweight and obesity prevalence rates. In addition, since the cross-sectional design does not allow any inferences regarding causality, we cannot ascertain whether certain child factors actually evoke the parenting practices, or whether parenting practices influence these child factors. Another possible limitation is that all data were reported by the parents of the children, and could therefore be biased, and that the measures of eating style, parenting practices and dietary intake were not validated. A fourth limitation is that the current study took only restrictive parenting practices into account. Other practices, like encouragement to eat healthy food and instrumental and emotional feeding might reinforce the positive influence of the restrictive practices found in this study. Moreover, these other parenting practices may interact with child characteristics in such a way that they have a greater impact on the children for whom restriction was found to have less impact, such as depressed or anxious children or slow eaters.

\section{Practical implications and further research}

The current research has shown that restrictive parenting practices regarding dietary intake cluster, and that these clusters seem to have a wide-ranging, favourable influence on children's specific dietary behaviours. This knowledge helps to identify a more general dietary and child rearing view among parents, and can provide valuable insights for preventive efforts, which are not obtained when focussing on individual parenting practices. The favourable influence of restrictive parenting practice clusters seemed to be more pronounced in children with 'favourable' characteristics. This indicates that these children in particular can gain a great deal from interventions focussing on diet-related parenting practices. Future interventions should focus on teaching parents effective parenting practices, even before the child reaches the age of 2 . This approach is in line with current increased attention for parent guidance in child rearing, stressed by professional organisations such as the American Academy of Pediatrics (Krebs \& Jacobson, 2003). In 
addition, we hypothesise that children who possess characteristics associated with lower effectiveness of restrictive parenting practices (e.g., higher BMI, anxiousness, depressiveness, overactivity, slow or reluctant eating style, not liking many foods) may benefit from more empathic diet-related parenting practices such as support and encouragement.

The results of the present study suggest that preventive interventions targeted at parenting practices, but tailored to individual child characteristics, could be effective. This hypothesis should be further examined in future research. In addition, longitudinal and experimental research is needed to examine the interactive nature of the relationship between child behaviour and parenting practices. 


\section{Chapter 5: Impact of parenting practices on children's dietary intake, physical activity and body mass index: the KOALA Birth Cohort Study}

This chapter has been submitted for publication as: Gubbels, J.S., Kremers, S.P.J., Stafleu, A., de Vries, S.I., Goldbohm, R.A., Dagnelie, P.C., de Vries, N.K., van Buuren, S. \& Thijs, C. (Submitted). Impact of parenting practices on children's dietary intake, physical activity and body mass index: the KOALA Birth Cohort Study. 


\begin{abstract}
Background: Insight into the effects of energy balance-related parenting practices on diet and physical activity at an early age is warranted in order to determine which practices should be recommended, and to whom. The purpose of this study was to examine predictors of energy balance-related parenting practices at age 5, as well as the associations of these practices with children's diet, activity and body mass index (BMI) development.

Methods: Questionnaire data originated from the KOALA Birth Cohort Study at ages $5(\mathrm{~N}=2026)$ and $7(\mathrm{~N}=1819)$. Linear regression analyses were performed to examine the association of child and parent background characteristics with parenting practices (i.e., diet- and physical activity-related restriction, monitoring and stimulation), and to examine the influence of these parenting practices on children's diet (i.e., energy intake, dietary fibre intake, added sugar intake) and activity (i.e., physical activity, sedentary time) at age 5, and BMI development from age 5 to age 7 . Moderation analyses were used to examine whether the influence of the parenting practices was dependent on child characteristics.

Results: Several child and parent background characteristics were associated with the parenting practices. Dietary monitoring, stimulation of healthy intake and stimulation of physical activity were associated with desirable energy balancerelated behaviours (i.e., dietary intake and/or physical activity) and desirable BMI development, whereas restriction of sedentary time showed undesirable effects. Child eating style and weight status, but not child gender or physical activity style, moderated the influence of the parenting practices on behaviour. Dietary restriction and monitoring seemed to be less effective or even had undesirable effects in children with a deviant eating style, while these practices had desirable effects in normal eaters. By contrast, stimulation to eat healthy worked particularly well for children with a deviant eating style or a high BMI.

Conclusion: Although most energy balance-related parenting practices were associated with desirable behaviours, there are also practices that influenced children's behaviour and weight outcomes negatively. Only parental stimulation was effective with regard to both diet and physical activity. The interaction between parenting and child characteristics in influencing behaviour calls for parenting that is tuned to the individual child.
\end{abstract}




\section{Background}

Eating and physical activity (PA) habits find their origin in early childhood (Reilly et al., 2004; Savage et al., 2007), and track into later life (Kelder et al., 1994; Patterson, Warnberg, Kearney, \& Sjostrom, 2009). Parents can have a strong influence on their child's dietary intake and PA: they can control the availability of and exposure to food and activity opportunities, they act as role models, provide their child with support and structure, and use specific parenting practices (Golan \& Crow, 2004). Parenting practices are content-specific acts of parenting (Darling \& Steinberg, 1993), such as rules about dietary intake or activity behaviour.

Many studies have aimed to examine the influence of food-related parenting practices and feeding styles on children's dietary intake and weight. Restriction of intake of unhealthy food items, for instance, has been found to be associated with a higher intake of those items and with a higher body mass index (BMI; see e.g., Clark, Goyder, Bissell, Blank, \& Peters, 2007; Faith, Scanlon et al., 2004; Rhee, 2008). However, there are also studies that show desirable effects of restriction on dietary intake (e.g., De Bruijn et al., 2007; Gubbels et al., 2009). Fewer studies examined diet-related parenting practices other than restriction, and also show inconsistent results (Faith, Scanlon et al., 2004). Promotion, stimulation or pressure to eat certain foods have been reported to have both favourable (e.g., Sleddens, Kremers, De Vries, \& Thijs, 2010; Vereecken, Keukelier, \& Maes, 2004; Zabinski et al., 2006) as well as unfavourable (e.g., Birch, Savage, \& Ventura, 2007) effects on children's diet, and were negatively associated with children's BMI (e.g., Powers, Chamberlin, van Schaick, Sherman, \& Whitaker, 2006). Conflicting findings have also been found with regard to monitoring of children's dietary intake, which was reported to be associated with childhood overweight (e.g., Moens, Braet, \& Soetens, 2007), but also with a lower child BMI (e.g., Faith, Berkowitz et al., 2004) and a healthier diet (Arredondo et al., 2006). A reason for these conflicting findings might be that many studies are cross-sectional, which makes it difficult to interpret results.

Less studies have been executed towards the effects of parenting practices concerning PA. Parental support and encouragement to be active seem to be important positive predictors of children's PA (Sallis, Prochaska, \& Taylor, 2000), although there are also many studies that do not find an effect on PA (Ferreira et al., 2007). Explicit rules about television watching have been found to be associated with less sedentary behaviour (e.g., He, Piche, Beynon, \& Harris, 2010; Salmon, Timperio, Telford, Carver, \& Crawford, 2005; Spurrier, Magarey, Golley, Curnow, \& Sawyer, 2008), but also with lower levels of PA in boys and higher levels of PA in girls (e.g., Salmon et al., 2005). Monitoring of a child's activity has been found to be associated with increased PA (Arredondo et al., 2006).

Examining the effect of different parenting practices on energy balancerelated behaviours is important in order to ascertain which practices should be 
recommended to parents in the prevention of childhood obesity. Moreover, there is an additional need to assess to whom these practices should be recommended, implying research regarding the association between background characteristics and parenting practices. For example, the use of diet-related controlling parenting practices, including restriction, has been shown to be related with parental BMI, educational level, age, ethnicity and employment (Arredondo et al., 2006; Brown et al., 2008; Gubbels et al., 2009; Hupkens et al., 1998; Liem et al., 2004; Montgomery et al., 2006). Pressure to eat was found to be related to parental ethnicity and socioecomic status, and diet monitoring to maternal age and BMI (Brown et al., 2008; Keller et al., 2006; Kroller \& Warschburger, 2009). Not only parental characteristics appear to be related to specific parenting practices, but also child characteristics have been found to evoke different parenting practices. For example, controlling practices, encouragement and monitoring have all been found to be correlated with children's weight status (e.g., Brann \& Skinner, 2005; Kroller \& Warschburger, 2009; Stang et al., 2004; Wardle \& Carnell, 2007), and controlling practices were related to the child's gender (Tiggemann \& Lowes, 2002). It is unclear which background factors predict the use of PA-related parenting practices, although a study in a Latino sample showed that employment of parents is associated with more control over the child's PA (Arredondo et al., 2006).

It has been advocated that there is an urgent need to know whether the effects of parenting practices are similar across distinct groups of children (e.g., Faith, Berkowitz et al., 2004; Gubbels et al., 2009). Insight in this question calls for moderation research (Kremers, 2010). Several child characteristics may moderate the effects of diet-related parenting practices. Dietary restriction and control were reported to have stronger undesirable effects on dietary intake of girls than of boys (e.g., Arredondo et al., 2006; Faith, Scanlon et al., 2004), but studies regarding moderation of gender in the relationship between restriction and weight status showed mixed results (Faith, Scanlon et al., 2004). Recently, we reported that restriction had a desirable impact on dietary intake of normal weight children, but not on dietary intake of overweight children (Gubbels et al., 2009). Furthermore, the desirable effect of restriction on dietary intake of 2-year-olds, was found to be less strong or even absent in children with a problematic eating style (i.e., who do not like many foods, eat with reluctance, or are slow eaters; Gubbels et al., 2009). Again, empirical evidence regarding moderators of parental influences on child PA is generally lacking, but in line with findings regarding the dietary intake domain, we would expect similar interactions of parenting practices with child factors within the PA domain.

The present study examined parental and child associates of energy balance-related (i.e., diet-related and PA-related) parenting practices. In addition, we examined the influence of energy balance-related parenting on physical activity and dietary intake in 5-year-old children, as well as the prospective influence of 
these practices on child's BMI development up to age 7. Finally, we examined whether child background characteristics moderated the impact of the parenting practices (see Figure 5.1).

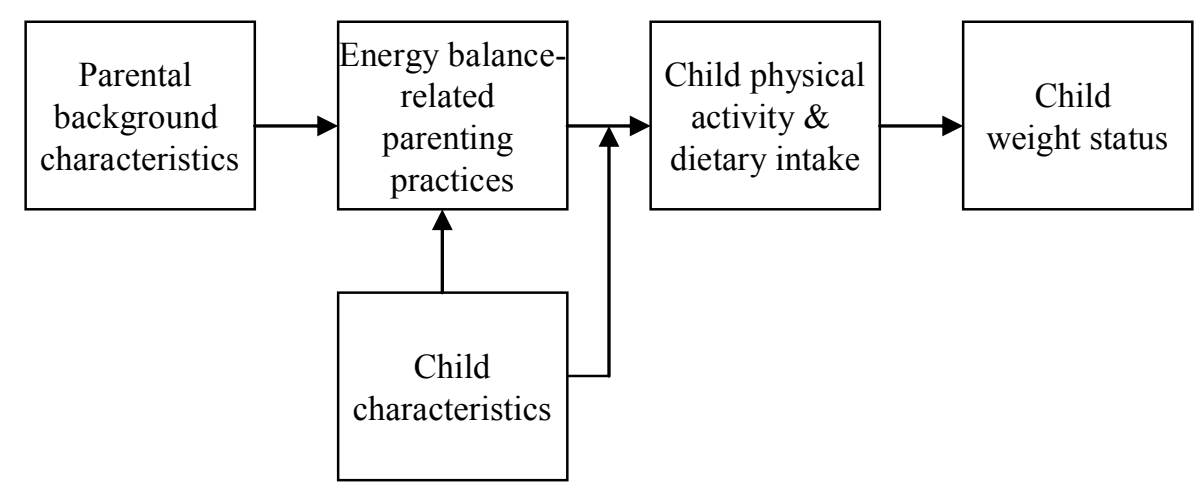

Figure 5.1: Model of energy balance-related parenting

\section{Methods}

\section{Respondents and procedure}

The KOALA Birth Cohort Study (the Netherlands) is a prospective cohort study which started in the year 2000. Healthy pregnant women were recruited from an existing cohort for a study of the aetiology of pregnancy-related pelvic girdle pain, as well as through recruitment channels among 'alternative lifestyle' circles (e.g., anthroposophist midwives and general practitioners, organic food shops; Kummeling et al., 2005). The latter group of women (17.9\%) could have an alternative lifestyle in terms of dietary habits (e.g., preferring organic foods), child rearing, vaccination schemes or antibiotics use. All participants signed informed consent, and approval was obtained from the Maastricht University/University Hospital Maastricht medical ethics committee. In total, mothers of 2834 children participated and completed questionnaires during pregnancy and regularly after birth. Ten children were excluded because of congenital defects (e.g., Down's syndrome).

\section{Questionnaires}

When the children were around 5 years old, parents completed a questionnaire regarding their energy balance-related parenting practices, their child's dietary intake, PA, weight and height, and several other child and parent characteristics. A total of 2026 questionnaires (71.7\%) were returned. At age 7, a follow-up 
questionnaire was sent, assessing weight and height, of which questionnaires regarding 1819 (89.8\%) children were returned.

\section{Child background characteristics}

The child's eating style was assessed on two dimensions: child's picky eating (Birch et al., 2001) and child's appetite. We also assessed whether the child had an active PA style. For more information about these concepts see Table 5.1. In addition, child's birth weight (in grams) and gender were also used in the current study.

\section{Parental background characteristics}

The questionnaire assessed working hours per week of father and mother, their educational level and their country of birth. Educational level was recoded into three levels (low, medium and high), in line with international classification systems (Eurostat, 2007). Country of birth was recoded into 'Netherlands' versus 'other'. In addition, maternal age at birth of the child was used in the current study.

\section{$\underline{\text { Parenting practices }}$}

We assessed parenting practices regarding children's dietary intake and PA. The items used to assess these parenting practices and the corresponding Cronbach's $\alpha$ 's can be found in Table 5.1. The parenting practices 'restriction' of unhealthy intake and 'monitoring' were assessed using the validated scales of the Child Feeding Questionnaire (CFQ; Birch et al., 2001), translated into Dutch. The current study focused on parenting practices in relation to weight gain prevention, and in this view 'stimulation of healthy intake' was considered more suitable than the original 'pressure' to eat scale of the CFQ. Pressure to eat is a practice which is often used to increase children's weight (Carper, Orlet Fisher, \& Birch, 2000). In addition, we 'converted' the diet-related items of the CFQ to the PA context in order to create a 'Physical Activity-related Parenting Questionnaire', consisting of three scales similar to the diet-related CFQ scales: 'restriction of sedentary behaviour', 'monitoring of physical activity' and 'stimulation to be physically active'.

\section{Child dietary intake, physical activity and BMI}

Children's dietary intake was assessed using a Food Frequency Questionnaire (FFQ), assessing intake during the 4 weeks preceding the questionnaire. This FFQ was especially developed to assess energy intake in children, and was validated using doubly labelled water (Dutman et al., 2010). The FFQ consisted of 71 items. For 27 foods additional questions were asked about the specific types or brands consumed and preparation methods. Parents indicated their child's habitual consumption frequency of each of the food items by checking 1 of 6 frequency categories ranging from 'never' to '6-7 days a week'. Portion sizes were asked in natural units (e.g., pieces, slices), household units (e.g., glasses, spoons) or grams 
(e.g., grams of meat). Parents were asked to measure the volume of the cups and glasses they used for the children. For the calculation of average energy intake (kJ) and fibre intake (in grams per MJ) per day, the Netherlands Food Composition (NEVO) Table 2001 was used (Stichting Nederlands Voedingsstoffenbestand, 2001). Nutritional values of products that were not (yet) included in the NEVO Table 2001, were provided by a dietician. In order to calculate added sugar intake (expressed as a percentage of total energy intake), which is not included in the NEVO Table, we used values from an earlier study (Balder, Ter Doest, \& Hulshof, 2006). Added sugar was defined as the amount of sacharose, glucose and/or fructose added to a food or meal by the consumer or the manufacturer.

Children's PA was assessed using questions based on a standard questionnaire for measuring PA, used in Dutch Youth Health Care (Monitor Jeugdgezondheid, 2009). Parents were asked how many days in a normal week during the last 4 weeks their child went to school by foot or bicycle, played sports at school (e.g., physical education lessons), played sports outside school at a sports club, and played outside (outside school hours). A second item assessed the average duration of each of these activities. The duration and number of days were multiplied to calculate the number of minutes spent in that activity per week. The minutes spent in the various activities were then summed to calculate the total number of minutes of physical activity per week, which was divided by 7 in order to get the average time (in minutes) being physically active per day. Sedentary screen-based behaviour was assessed in a similar manner, asking parents about their child's television watching (including videos and DVDs) and computer playing.

Parents were asked to report their child's weight and height (measured without shoes and cloths, specified to one decimal), in order to calculate the child's body mass index (BMI, i.e., weight $\left.(\mathrm{kg}) /(\text { height }(\mathrm{m}))^{2}\right)$. BMI was then recoded into BMI z-scores compared to the national reference population of 1997 (i.e., the Fourth Dutch National Growth Study; Fredriks et al., 2000). At age 7, parents were asked to report their child's height and weight again, and these were recoded into BMI z-scores at the age of 7 years.

\section{Data analyses}

The analyses were conducted using SPSS 15.0. Cronbach's $\alpha$ 's were calculated as an estimate of the lower bound of reliability of the scales used (e.g., the CFQ scales). All analyses described below were adjusted for recruitment group (alternative versus conventional), and $\mathrm{p}$-values $<.05$ were considered statistically significant.

First, linear regression analyses were used to examine predictors of the use of the six energy balance-related parenting practices (i.e., restriction of intake, monitoring of intake, stimulation of healthy intake, restriction of sedentary time, monitoring of PA, and stimulation to be active). The predictors that were examined 
were child background characteristics (child gender, birth weight, BMI z-score at age 5, PA style, hungry eating style and picky eating style) and parental background characteristics (parental BMI, employment, educational level, and country of birth; maternal age). All predictors were entered simultaneously, correcting for potential confounding of the other variables.

Second, linear regression models were fitted to assess the associations of the parenting practices with each of the six outcome variables: energy intake, fibre intake, added sugar intake, PA, and sedentary behaviour (all at age 5), and BMI zscore at age 7. The diet-related parenting practices were included in the analyses with the dietary intake variables as the outcome, and the PA-related parenting practices were included in the analyses with PA and sedentary time as an outcome. Both diet-related and PA-related parenting practices were included in the analyses examining the influence on BMI at age 7. These analyses were adjusted for the child characteristics (including BMI z-score at age 5) and parental background characteristics described above. The analyses with BMI z-score at age 7 as the dependent variable were repeated excluding children that were underweight (BMI $\mathrm{z}$-score $<5^{\text {th }}$ percentile) at age 5 , to examine whether this resulted in the same findings.

Third, in order to examine whether child characteristics moderate the influence of the parenting practices on energy balance-related behaviour and BMI development, we calculated interaction terms between the parenting practices and child characteristics. The interaction terms were added to the regression analyses in a separate step using stepwise forward entering. With this forward procedure, the interaction term that had the highest correlation with the unexplained variance of the outcome variable was added to the model, on condition that it significantly improved the predictive value of the model. This procedure was repeated until no significant improvement to the predictive value of the model could be made anymore by any of the interaction terms that was not yet included in the model (Field, 2005). Subsequently, stratified linear regression analyses were performed for the interaction terms that were included in the model in this separate step, in order to examine the effect of the parenting practice in the different strata of the moderator variable (i.e., the child characteristic). Continuous variables were dichotomized for this purpose, using a median split. We only report the interactions for which the effect of the parenting practice was statistically significant in either or both of the strata of the moderator variable.

\section{Results}

Of the 2026 children participating at the questionnaire around age 5, 51.2\% were male. The mean daily energy intake of the children was $6176 \mathrm{~kJ}(1467 \mathrm{kCal})$, with a standard deviation (SD) of $1286 \mathrm{~kJ}$ (306 kCal). The children consumed on average 2.5 grams of dietary fibre per MJ of energy intake $(\mathrm{SD}=.6)$, and added sugar intake 
contributed $15.8 \%(\mathrm{SD}=6.6 \%)$ to their total energy intake. Children were physically active for an average of 116 minutes per day $(\mathrm{SD}=55)$, and spent 59 minutes in sedentary screen-based activities $(\mathrm{SD}=42)$. Mean BMI z-score at age 5 was -.27 $(\mathrm{SD}=.99)$, at age 7 this was $-.29(\mathrm{SD}=.94)$. Descriptive information regarding children's eating styles and PA style can be found in Table 5.1. Mean maternal BMI was $24.0 \mathrm{~kg} / \mathrm{m}^{2}(\mathrm{SD}=3.8)$, mean paternal BMI was $25.0 \mathrm{~kg} / \mathrm{m}^{2}(\mathrm{SD}=3.1)$. Mothers worked 18.0 hours $(\mathrm{SD}=11.1)$ and fathers worked 37.8 hours $(\mathrm{SD}=10.1)$ per week on average. A total of $3.0 \%$ of the mothers and $3.7 \%$ of the fathers had not been born in the Netherlands. Educational level was high for $54.1 \%$ of the mothers and $53.2 \%$ of the fathers, medium for $38.0 \%$ and $33.9 \%$, and low for $7.9 \%$ and $12.9 \%$, respectively. Average maternal age at birth of their child was 32.2 years $(\mathrm{SD}=3.7)$.

\section{Predictors of parenting practices}

Several child characteristics were significantly related to the used parenting practices at age 5 years (Table 5.2). Parents imposed more dietary restriction on girls than on boys. The reverse was true for restriction of sedentary time: boys were more restricted by their parents than girls. A higher child BMI z-score (at age 5) was associated with more dietary restriction and more stimulation of healthy intake. Dietary intake was more restricted by parents whose child had a hungry or picky eating style. Further, children who were picky eaters were less often stimulated to eat healthy. Children with an active PA style were less often restricted in their sedentary time, but also more often stimulated to be active than their normal peers.

Various maternal characteristics were important predictors of the parenting practices (Table 5.2). A higher maternal BMI was associated with less restriction and less stimulation with regard to dietary intake. Maternal educational level was positively associated with stimulation of healthy intake, stimulation to be physically active and restriction of sedentary time, while mothers' working hours were negatively related to monitoring of both dietary intake and PA, and to stimulation to be physically active. Paternal educational level was inversely associated with monitoring of dietary intake. 


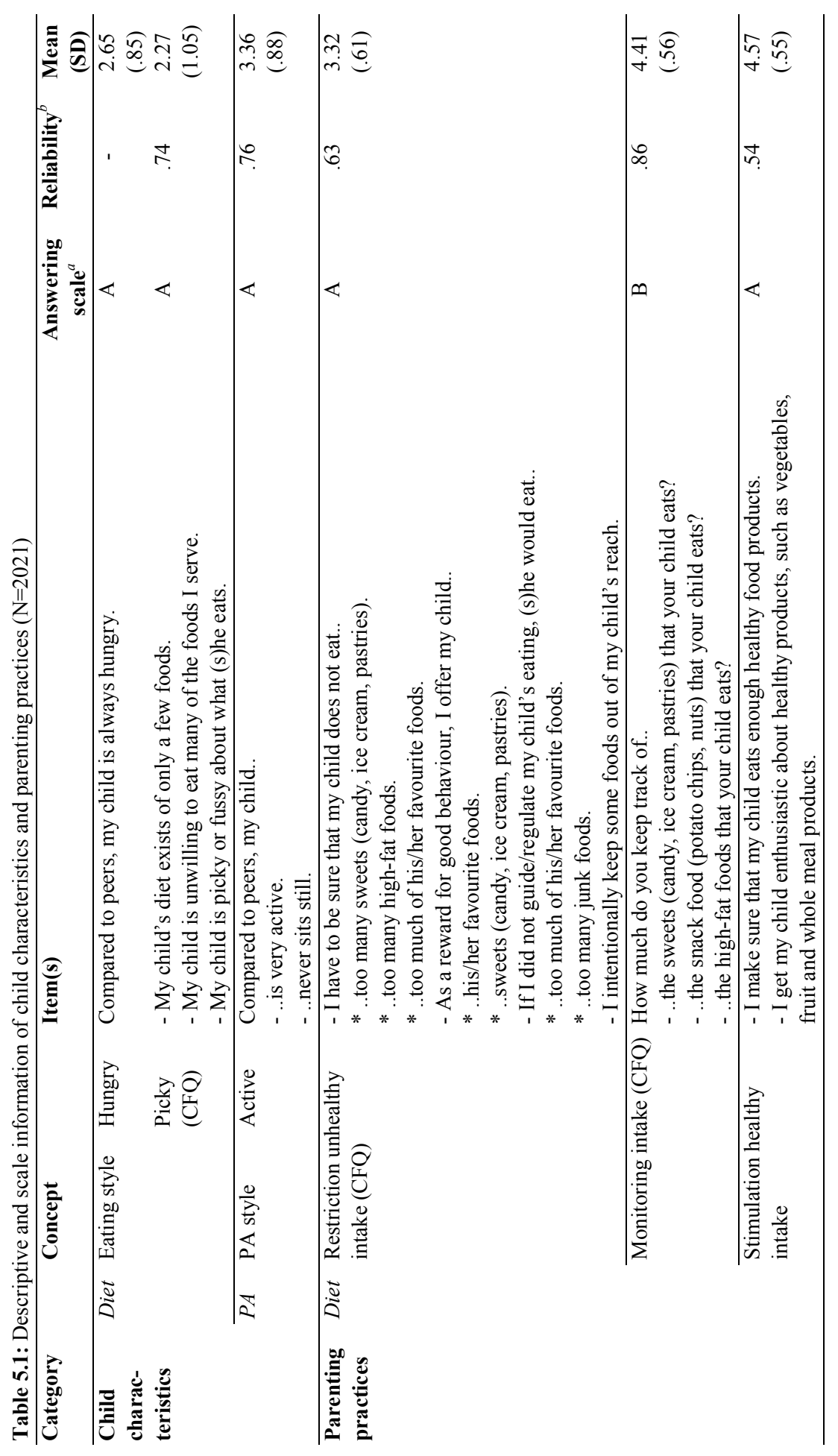




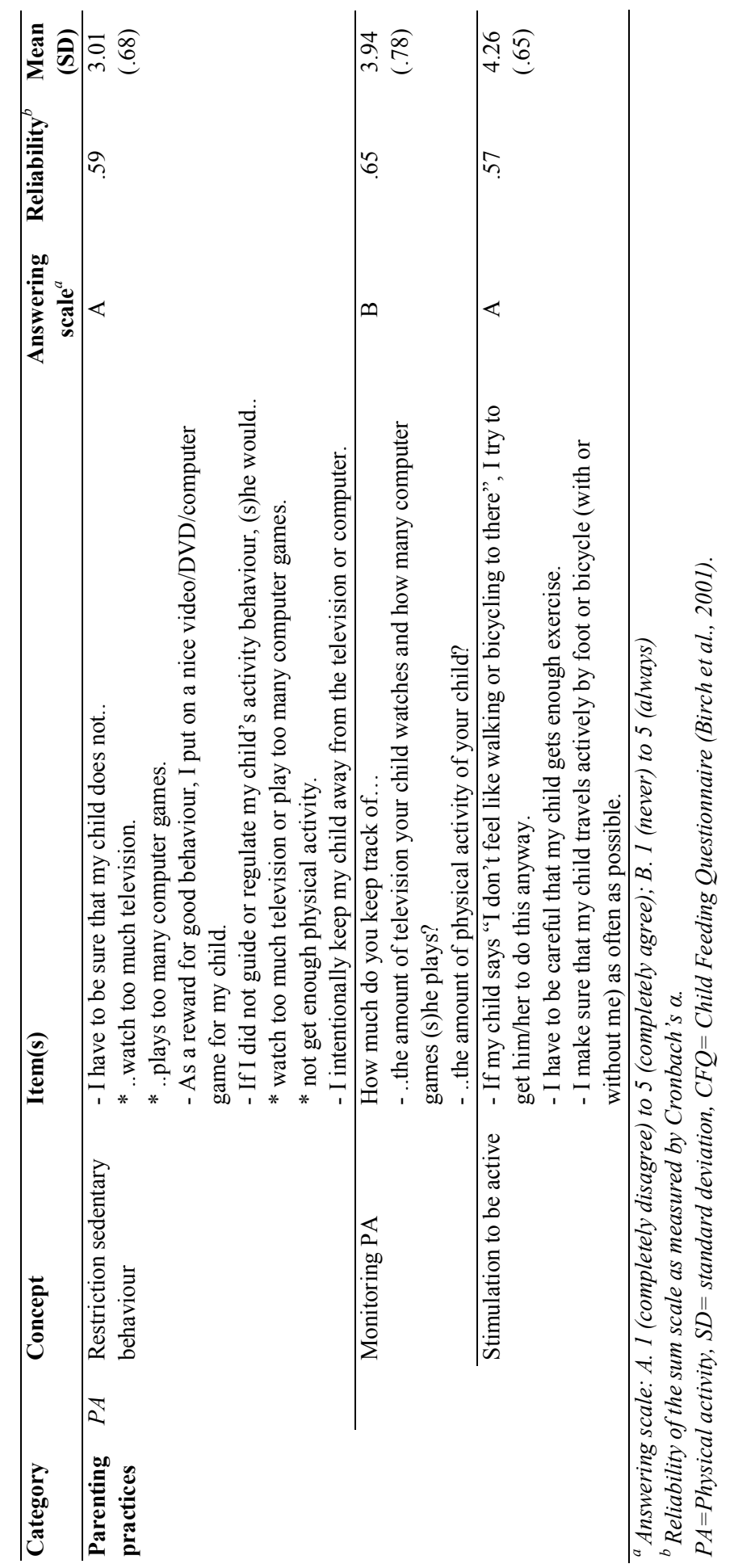




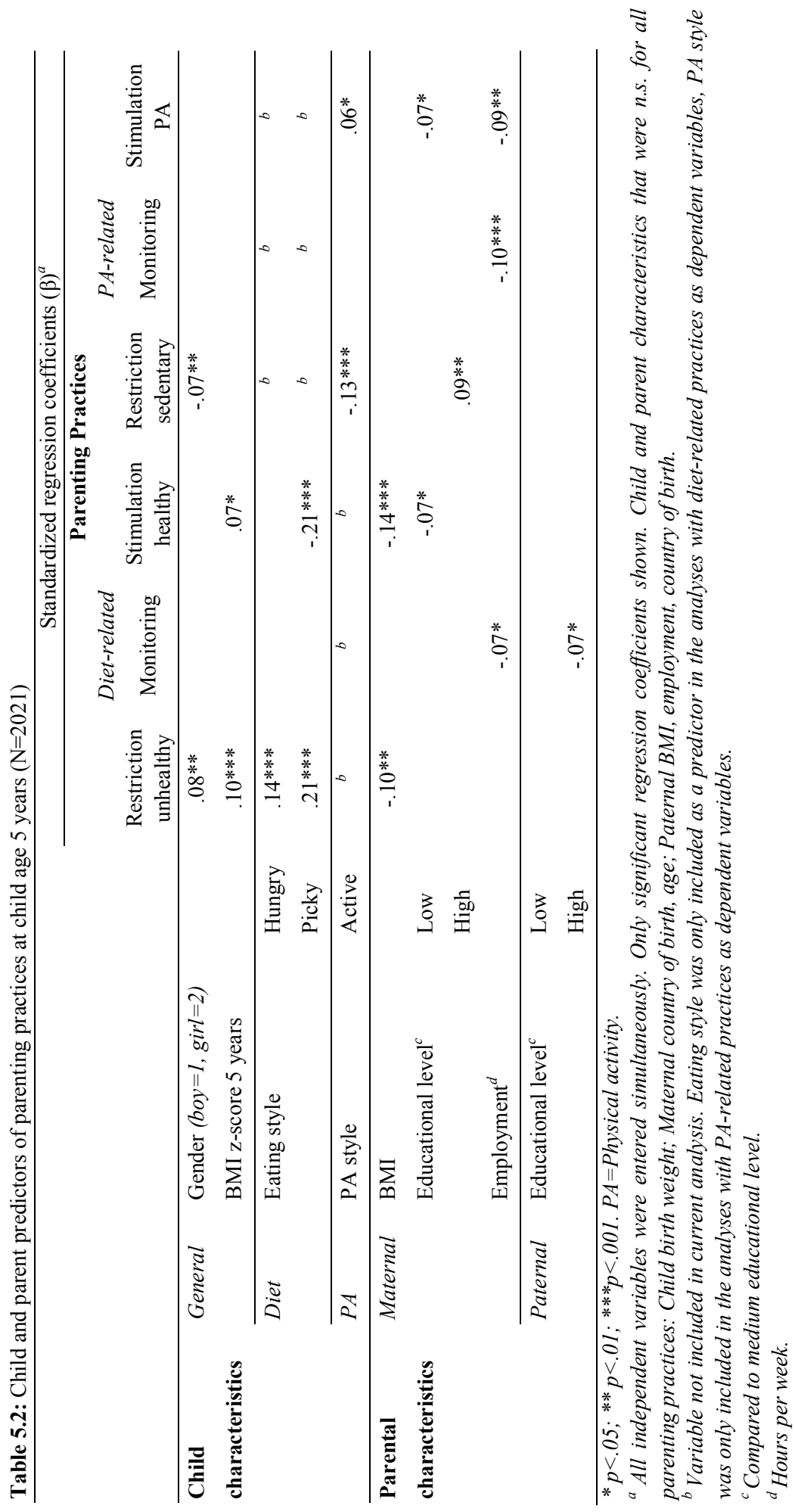




\section{Influence of parenting practices on diet and $P A$ at age 5}

Stimulation of healthy intake and monitoring of a child's diet were not only associated with more dietary fibre intake, but also with less added sugar intake (Table 5.3). Children's energy intake was not associated with the parenting practices. Restriction of sedentary behaviour was related to higher sedentary time and less PA. Stimulation to be active was positively associated with PA, and negatively with sedentary behaviour.

Table 5.3: Association of parenting practices at age 5 with child's dietary intake and physical activity at 5 years $(\mathrm{N}=2021)$

\begin{tabular}{|c|c|c|c|c|c|}
\hline & \multicolumn{5}{|c|}{ Standardized regression coefficients $(\beta)^{a}$} \\
\hline Parenting practice & $\begin{array}{c}\text { Energy } \\
\text { intake } \\
(k J / d a y)\end{array}$ & $\begin{array}{c}\text { Fibre } \\
\text { intake } \\
(g / M J) \\
\end{array}$ & $\begin{array}{c}\text { Sugar } \\
\text { intake } \\
(E n \%) \\
\end{array}$ & $\begin{array}{c}\text { Physical } \\
\text { activity } \\
(\mathrm{min} / \mathrm{day})\end{array}$ & $\begin{array}{r}\text { Sedentary } \\
\text { behaviour } \\
(\mathrm{min} / \mathrm{day})\end{array}$ \\
\hline $\begin{array}{ll}\text { Diet } & \text { Restriction } \\
& \text { unhealthy intake }\end{array}$ & -.03 & -.01 & -.04 & - & - \\
\hline Monitoring intake & -.02 & $.09 * *$ & $-.08 * *$ & - & - \\
\hline $\begin{array}{l}\text { Stimulation healthy } \\
\text { intake }\end{array}$ & .01 & $.18 * * *$ & $-.07 * *$ & - & - \\
\hline $\begin{array}{ll}\text { PA } & \text { Restriction } \\
& \text { sedentary time }\end{array}$ & - & - & - & $-.19 * * *$ & $.09 * *$ \\
\hline Monitoring $P A$ & - & - & - & .03 & -.03 \\
\hline Stimulation PA & - & - & - & $.12 * * *$ & $-.12 * * *$ \\
\hline \multicolumn{6}{|c|}{$\begin{array}{l}\text { ** } p<.01 ; * * * p<.001 \text {. } \\
k J=\text { kilo Joules; } g / M J=\text { intake in grams from nutrient per } M J \text { of total energy intake; En\%=Energy } \\
\text { intake from nutrient as a percentage of total energy intake; min/day=minutes per day; PA=Physical } \\
\text { activity. } \\
\text { analyses were adjusted for recruitment group (alternative vs. conventional), child background } \\
\text { characteristics (gender, birth weight, BMI z-score at age 5, physical activity style and eating style) and } \\
\text { parent background characteristics (parental BMI, educational level, employment and country of birth; } \\
\text { and maternal age). }\end{array}$} \\
\hline
\end{tabular}

\section{Influence of parenting practices on BMI development from age 5 to age 7}

All analyses regarding BMI at age 7 were corrected for BMI at age 5, so that BMI results at age 7 reflect BMI development from 5 to 7 years of age. Stimulation of healthy intake was negatively associated with BMI development up to age 7, while restriction of sedentary time was positively related to BMI development (Table 5.4). Repeating the analyses while excluding children that were underweight at age 5 $(\mathrm{N}=187)$ did not yield substantially different results. 
Table 5.4: Association of parenting practices at age 5 with child's BMI development up to age 7

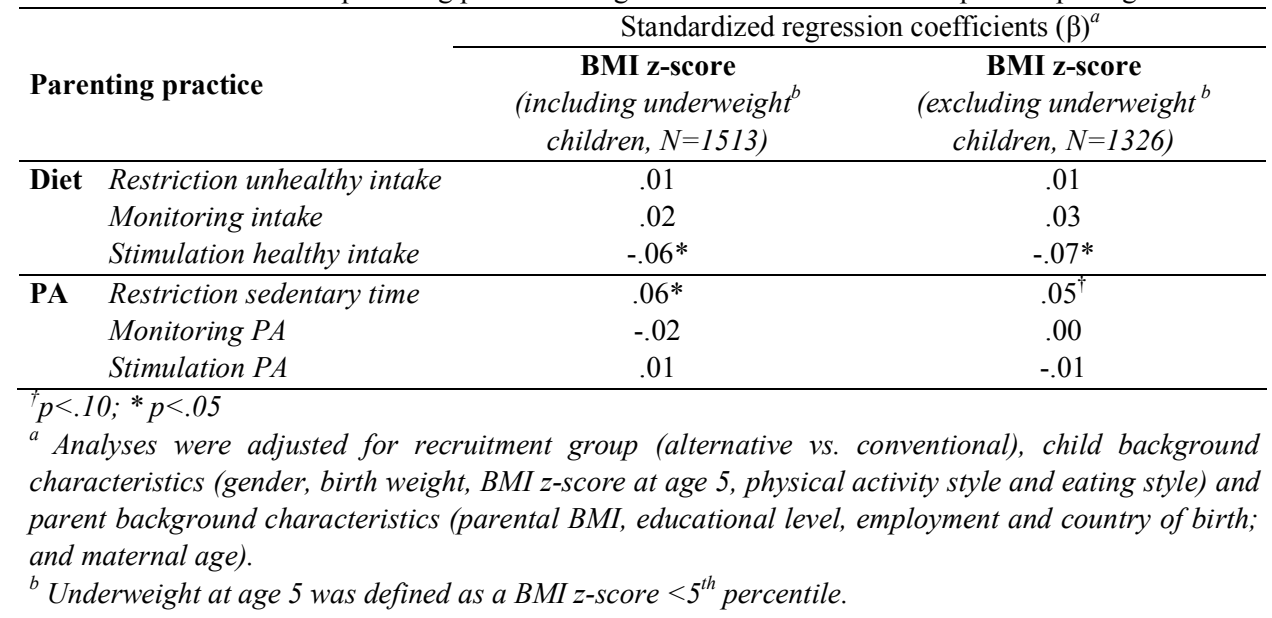

\section{Child background characteristics as moderators of parenting practice impact}

There were several significant interactions between parenting practices and child background characteristics. The impact of the diet-related practices on children's dietary intake was found to be moderated by the child's eating style and weight status. Restriction was associated with increased energy intake in children who were characterized as relatively hungry (standardized $\beta=.18, \mathrm{p}<.05$ ), while this was not the case for their peers with a normal appetite $(\beta=-.05$, non significant (n.s.)). The desirable effects of diet monitoring on dietary fibre and added sugar intake were absent in hungry children and picky eaters (absolute $\beta$ 's $<.05$, n.s.). These desirable effects were, however, present in children that were not indicated to be relatively hungry ( $\beta=.11, \mathrm{p}<.001$ for fibre intake; $\beta=-.09, \mathrm{p}<.01$ for sugar intake), and were not indicated to be a picky eater $(\beta=-.13, \mathrm{p}<.01$ for sugar intake). By contrast, the desirable effect of stimulation of healthy intake on added sugar intake was present in picky eaters $(\beta=-.17, p<.001)$, but not in normal, non-picky, eaters $(\beta<.01$, n.s.). Furthermore, stimulation of healthy intake had a slightly stronger desirable effect on fibre intake in children with a BMI above the median $(\beta=.22$, $\mathrm{p}<.001)$, compared to children with a lower BMI at age $5(\beta=.15, \mathrm{p}<.001)$. There were no interactions found between diet-related practices and the child's gender.

Regarding physical activity, the desirable effect of stimulation to be active on PA was only present in children with birth weight below the median $(\beta=.19$, $\mathrm{p}<.001$ ), and not in children with a higher birth weight ( $\beta=.04$, n.s.). There were no interactions between PA-related practices and the child's gender or PA style. 


\section{Discussion}

The current study examined child and parent predictors of energy balance-related parenting practices, as well as the effects of those practices on diet and PA at age 5, and BMI development from age 5 to 7 years. Parents were found to be more restrictive regarding their daughters' diet than of their sons', which is in line with previous research (Tiggemann \& Lowes, 2002). However, the current study also showed that girls were less restricted than boys when it came to sedentary time. Possibly parents have different priorities in boys and girls when it comes to restricting unhealthy behaviours, and perhaps inactivity is more of a concern for parents for their sons, while overconsumption is of more concern to parents for their daughters. Parental restriction was further positively associated with child BMI, in accordance with previous studies (Stang et al., 2004).

There were also several parental characteristics that predicted which practices parents applied. Maternal BMI was found to be inversely associated with dietary restriction and stimulation, which confirms previous findings (Brown et al., 2008; Liem et al., 2004; Montgomery et al., 2006). Maternal educational level was positively related with stimulation of both healthy intake and PA. This adds to previous research that showed that parental education was positively associated with restriction and other controlling practices (Brown et al., 2008; Gubbels et al., 2009; Hupkens et al., 1998). The number of hours mothers worked was negatively associated with monitoring of diet and PA and stimulation to be active. A similar association was previously reported by Brown and colleagues (2008), showing that parents who stayed at home to take care of their children were more controlling of their children's diet. As working parents leave part of child rearing to others, such as child-care staff (see e.g., Gubbels et al., 2010), they may be inclined to be less strict during the limited time they can spend with their child.

With regard to the effects of the parenting practices on their children, we found that monitoring of a child's diet and stimulating healthy intake were both associated with favourable effects on children's diet. Stimulation of healthy intake even had a desirable effect on the child's BMI development up to the follow-up at age 7. By contrast, dietary restriction was not associated with any of the dietary outcomes, nor was it associated with BMI development. Previous studies have shown conflicting results with regard to all three of the above mentioned parenting practices (i.e., monitoring, see e.g., Arredondo et al., 2006; Faith, Berkowitz et al., 2004; Moens et al., 2007; stimulation, see e.g., Birch et al., 2007; Sleddens et al., 2010; Vereecken et al., 2004; Zabinski et al., 2006; restriction, see e.g., Clark et al., 2007; De Bruijn et al., 2007; Faith, Scanlon et al., 2004; Gubbels et al., 2009; Rhee, 2008), with studies both supporting as well as studies opposing the current findings. We believe the key to resolving these conflicting findings might lie in the interaction between child and parents. In line with hypotheses based on previous studies (see e.g., Faith, Berkowitz et al., 2004; Gubbels et al., 2009), the current 
study showed that the effects of parenting practices were dependent on characteristics of the child. Dietary restriction was associated with undesirable dietary intake behaviours in children with a deviant eating style (i.e., children that were relatively hungry compared to peers). In line with this, previous research showed that the desirable effects of restriction at a very young age ( 2 years), were partly absent in children with deviant eating styles (Gubbels et al., 2009). Analogous to the findings with regard to restriction, the desirable effects of monitoring were found to be absent in relatively hungry children and picky eaters. By contrast, stimulation to eat healthy was found to be specifically beneficial for picky eaters, as well as for children with a high BMI. This indicates, in line with previously raised hypotheses (Gubbels et al., 2009), that although restriction and monitoring might be less suitable for children with certain unfavourable characteristics (e.g., deviant eating style, high BMI), stimulation to eat healthy seems all the more effective for these children. It is worrisome however, that picky eating was also a predictor of less parental stimulation to eat healthy. Educating parents might therefore be an important step to improve children's diet, perhaps especially for children with a deviant eating style.

The effects of rules about television viewing on PA have previously been found to depend on the gender of the child, with desirable effects in girls, but undesirable effects in boys (Salmon et al., 2005). We did not find indications of such a difference in the current study, but we found that restriction of sedentary time had undesirable effects for both boys and girls; restriction was associated with increased sedentary time, decreased PA, and an increased BMI development up to age 7. This contradicts previous studies that have shown that explicit rules restricting children's amount of television watching were associated with less television viewing (He et al., 2010; Salmon et al., 2005; Spurrier et al., 2008). Stimulation to be active showed a positive association with children's PA and a negative association with sedentary time in our study, which is in line with a review showing that encouragement and support are important predictors of increased PA (Sallis et al., 2000).

The findings of the current study have implications for both research and for practice. With regard to research, studies into the effects of parenting practices that do not incorporate the possibility of moderation by child characteristics, will tend to lead to conclusions on the effects of parenting practices that strongly depend on their study population. In addition to the moderators identified in the current study, previous research has revealed several additional child factors that moderate the effects of parenting practices, including child personality and temperament (De Bruijn et al., 2007; Gubbels et al., 2009), gender (Faith, Scanlon et al., 2004) and weight (Gubbels et al., 2009). These interactions might also be contributing to the many contradictions in the current diet-related parenting practices evidence base. Therefore, we believe that research into the effects of parenting practices cannot be limited to the direct association between practices and outcomes, but should always 
incorporate a theory-based examination of possible moderation effects (Kremers, 2010; Spence \& Lee, 2003). The practical implications of the current findings are that overweight prevention interventions targeted at parenting practices should be tailored to individual child characteristics, since specific parenting practices might be beneficial for one child, but useless (or even potentially disadvantageous) for another.

There are several limitations and strengths of the current research that need to be acknowledged. One of the strengths of the study is that a longitudinal followup for the effects on BMI development was included. However, behavioural outcomes were only assessed cross-sectionally. Therefore we cannot establish whether these behaviours are the consequence of certain parenting practices, or that they perhaps evoke these parenting practices. The same goes for the eating style and PA style, which we regarded as relatively stable child characteristics, and therefore included as predictors of parenting practices. They could, however, also be influenced by parenting practices. Many of the previous studies in this research area are limited to cross-sectional explorations, and there is a need for prospective research to establish causality (see e.g., Faith, Scanlon et al., 2004; Ferreira et al., 2007). It is reassuring, though, that the effects of two of the parenting practices (i.e., stimulation of healthy intake and restriction of sedentary time) on behaviour, were supported by the associations with later BMI development, pointing in the same direction. An additional strength is that the data in the current study were assessed prospectively, limiting the possibility of recall bias and other problems faced with retrospective research.

A major limitation of the current study is that all data, including dietary intake, PA and anthropometrics, were self-reported by the parents, possibly introducing bias. In addition, Cronbach's $\alpha$ 's of some of the scales were relatively low. Although Cronbach's $\alpha$ 's $\geq .6$ are generally considered acceptable (Nunnally $\&$ Bernstein, 1994), there are also authors stating different cut off points. Furthermore, caution is warranted when generalizing the results, as the current sample included an overrepresentation of parents with an 'alternative', relatively healthy lifestyle, due to the choice of recruitment methods (Kummeling et al., 2005). This is reflected in the relatively low mean BMI z-scores of the children. However, secondary analyses showed that excluding the children who where underweight at age 5 did not change our findings. Finally, it may be noted that the reported effect sizes are small, indicating that the explained variance in behaviour and weight status by the parenting practices is limited. This might be partly attributable to the fact that parenting behaviour is a concept that is hard to assess, and there is no consensus about the proper way to measure it. There are dozens of questionnaires assessing diet-related parenting practices (see e.g., Baughcum et al., 2001; Birch et al., 2001; Musher-Eizenman \& Holub, 2007). With regard to physical activity-related parenting practices there are not even any validated or widely accepted questionnaires available. We feel quite confident, though, with the 
instruments adapted form the CFQ (Birch et al., 2001) for the current study. The finding that the adapted scales 'stimulation of healthy intake' and 'restriction of sedentary time' predicted BMI change from age 5 to age 7 may be considered reassuring in this respect. Future research would benefit from reaching consensus about feasible and valid measurement methods.

\section{Conclusions}

In conclusion, the current study showed that although most energy balance-related parenting practices were associated with desirable behaviours, there are also practices that influence 5-year-old children's behaviour and subsequent weight outcomes negatively (e.g., restriction of sedentary time). Stimulating a child seems to be an effective practice for establishing both a healthy diet as well as a healthy PA pattern in children. However, the effects of several of the parenting practices were shown to be dependent on child characteristics, which calls for parenting that is tuned to the individual child. 


\section{Part 2: Child-care environment}





\section{Chapter 6: Child-care use and the association with body mass index and overweight in children from 7 months to 2 years of age}

This chapter has been published as: Gubbels, J.S., Kremers, S.P.J., Stafleu, A., Dagnelie, P.C., de Vries, N.K, van Buuren, S. \& Thijs, C. (2010). Child-care use and the association with body mass index and overweight in children from 7 months to 2 years of age. International Journal of Obesity, Epub May $25^{\text {th }} 2010$. 


\begin{abstract}
Objectives: Studies regarding the association of child-care use with body mass index (BMI), overweight or obesity development show contradictory results. This study examined the relationship between child-care use and BMI z-scores and overweight, as well as associates of child-care use in children up to 2 years old.

Methods: Longitudinal data originated from the Dutch KOALA Birth Cohort Study. Questionnaires assessed child-care use at ages 7 months and 1 and 2 years $(\mathrm{N}=2396)$. Height and weight assessed at 1 and 2 years were used to calculate BMI z-scores. Overweight was defined as a BMI z-score $\geq 85^{\text {th }}$ percentile. The influence of child-care use on weight development was tested using backward linear and logistic regression analyses. Outcomes were: 1) BMI z-score at 1 and 2 years; 2) change in BMI z-score between 1 and 2 years; 3) overweight vs. non-overweight at 1 and 2 years; and 4) change from normal weight to overweight vs. remaining normal weight between 1 and 2 years. The association between child-care use and parental background characteristics was tested using backward logistic regression analyses.

Results: Child-care use (no/yes) at 1 and 2 years positively predicted BMI z-scores at age 2, as well as change in BMI z-score between 1 and 2 years. These associations were adjusted for various covariates (e.g., parental working hours). Furthermore, child-care use significantly increased the odds of being overweight at age 1. There were few differences in BMI or overweight between intensive $(>16$ hours per week) and limited child-care use ( $\leq 16$ hours). Child-care use was positively associated with various parental characteristics, including parental working hours and maternal educational level.

Conclusion: The findings suggest a small influence of child-care use on weight development in very young children. The child-care setting could play an important role in preventive interventions against overweight and obesity development in young children.
\end{abstract}




\section{Introduction}

Childhood overweight prevalence is increasing globally (World Health Organization, 2006). Overweight children are at risk for various chronic conditions, such as cardiovascular diseases and type 2 diabetes mellitus (World Health Organization, 2004). Moreover, they often develop into overweight or obese adolescents and adults (Johannsson, Arngrimsson, Thorsdottir, \& Sveinsson, 2006; Singh et al., 2008). Among the most important determinants of childhood overweight development are an unhealthy diet and low levels of physical activity (Rennie et al., 2005). Dietary and physical activity habits are often formed at a young age, and once established, are mostly maintained throughout life (Kelder et al., 1994; Reilly et al., 2004).

Child-care is a setting in which young children today spend increasing amounts of time. In Europe, a quarter of the infants and over three quarters of the children up to school age attend some form of child-care or education facilities (Blackburn, 2006; Eurostat, 2008). This setting could therefore potentially play an important role in the prevention of overweight and obesity development in young children (Story et al., 2006). Previous research has underlined the influence of the child-care setting on both dietary intake (Gubbels et al., 2010; Hughes et al., 2007) and physical activity among children (Bower et al., 2008; Pate, McIver, Dowda, Brown, \& Addy, 2008). Studies examining the relation between child-care use and overweight or obesity have, however, been scarce, and have shown mixed results. A recent study by Benjamin and colleagues (2009) found that adiposity at ages 1 and 3 years was significantly and positively related to child-care use during the first six months of life (Benjamin et al., 2009). Kim and Peterson (2008) found that childcare by a relative, but not centre-based or non-relative child-care, was associated with significantly more weight gain in the first nine months of life, compared to no child-care (Kim \& Peterson, 2008). Another study found that obesity at kindergarten entry was predicted by previous child-care use, with children attending at least 10 hours of child-care a week more often being overweight than those who attended child-care for less than 10 hours a week (Maher, Li, Carter, \& Johnson, 2008). In contrast, Lumeng et al. (2005) indicated that child-care use was protective against overweight and obesity (Frisvold \& Lumeng, 2007; Lumeng, Gannon, Appugliese, Cabral, \& Zuckerman, 2005), although this protective effect was only present with limited child-care use ( $<15$ hours a week). Extensive child-care use $(\geq$ 15 hours a week) did not result in a significant difference in overweight compared to no child-care use (Lumeng et al., 2005). Other studies found no significant relation between child-care use and either childhood or adult Body Mass Index (BMI) and overweight (Kimbro, Brooks-Gunn, \& McLanahan, 2007; Rapp, Schick, Bode, \& Weiland, 2005; Wronka \& Pawlinska-Chmara, 2007).

Child-care use, in turn, has been found to be predicted by several parental factors, including higher income (Lumeng et al., 2005), longer duration of 
employment (Zaslow, Oldham, \& Moore, 1998), and higher educational level (Huston, Chang, \& Gennetian, 2002; Wronka \& Pawlinska-Chmara, 2007; Zaslow et al., 1998). More child-care use was also associated with a more stimulating and supporting home environment (Lumeng et al., 2005; Zaslow et al., 1998). Other factors that predicted child-care use were residential environment (with more childcare use in towns and cities compared to villages; Wronka \& Pawlinska-Chmara, 2007), ethnicity (Lumeng et al., 2005) and family structure (less use of child-care with an increasing number of siblings; Huston et al., 2002; Wronka \& PawlinskaChmara, 2007; Zaslow et al., 1998). Because of these many associates of child-care use, disentangling the effect of child-care use on overweight development requires longitudinal analysis controlling for these background variables.

In the current study, we examined the longitudinal influence of child-care use on subsequent weight development up to the age of 2 years, controlled for various relevant covariates. Outcomes were: 1) BMI z-score at 1 and 2 years of age; 2 ) the change in BMI z-score between ages 1 and 2 years; 3 ) overweight vs. nonoverweight at age 1 and 2 years; and 4) change from normal weight to overweight vs. remaining normal weight between 1 and 2 years of age. In addition, we examined associates of child-care use in children aged between 7 months and 2 years, in order to identify which parents take their child to child-care instead of caring for them at home.

\section{Methods}

\section{Respondents and procedure}

The KOALA Birth Cohort Study is a prospective cohort study in the Netherlands, which started in the year 2000. KOALA is the Dutch acronym for Child, Parent and health: Lifestyle and Genetic constitution. Healthy pregnant women participating in a study of pregnancy-related pelvic girdle pain were recruited at 34 weeks gestation for the study (conventional recruitment group). In addition, women were recruited through 'alternative lifestyle' recruitment channels (e.g., anthroposophist midwives and general practitioners, Steiner schools and organic food shops; Kummeling et al., 2005). This alternative recruitment group (17.3\%) had an alternative lifestyle in terms of aspects like dietary habits (e.g., using organic foods) or child rearing, and we further refer to this group using the variable 'parental alternative lifestyle'. Written informed consent was obtained from all participants, and ethical approval was obtained from the Maastricht University/University Hospital Maastricht medical ethics committee. In total, 2834 women participated, and completed questionnaires during pregnancy and after their child was born. 


\section{Questionnaires}

Questionnaires at several ages were used to assess various characteristics of the children and their parents. In the current study, data of questionnaires at the ages of 7 months and 1 and 2 years were analysed. The total number of questionnaires returned was $2614(91.9 \%)$ at 7 months, $2566(90.2 \%)$ at 1 year, and $2578(90.6 \%)$ at 2 years. There were $2396(84.2 \%)$ children for whom questionnaires were returned at all three ages, and who were included in the analyses. Drop-out analyses were executed to examine selective drop-out regarding background characteristics (i.e., number of hours of employment and overweight of mother and of father; parental alternative lifestyle; maternal educational level, age and country of birth). Logistic regression analyses showed that participants that provided data at all three ages did not differ on any of these factors from participants that did not provide complete data.

\section{Child characteristics}

Child-care use at the ages of 7 months, 1 year and 2 years, was assessed by asking parents if their child attended formal child-care facilities outside the home, and if so, how many hours. Answering options were: no child-care attendance, 1-8 hours, $9-16,17-24,25-32,33-40$ or more than 40 hours per week. Child-care use was categorised in two ways. Children were first categorised into attending child-care (1) or not (0), and those attending child-care were then categorised into intensive (1) versus limited (0) use, using a median split ( $>16$ hour vs. $\leq 16$ hours per week). Informal child-care arrangements, such as child-care by a family member, were not included in this study.

Parents were asked to report the height and weight of their child at 1 and 2 years, as assessed during the periodical assessments at infant welfare centres, under the Dutch preventive health care system. Weight and height were used to calculate BMI (i.e., weight $(\mathrm{kg}) /(\text { height }(\mathrm{m}))^{2}$ ), which was then recoded into BMI z-scores compared to the national reference population (i.e., the Fourth Dutch National Growth Study, 1997; Fredriks et al., 2000). Children with a BMI $z$-score $\geq 85^{\text {th }}$ percentile were considered overweight (Barlow, 2007). In addition, children's absolute birth weight (in grams) and gender were recorded, and parents reported the duration of breast-feeding (in months) in the first year of the child's life.

\section{$\underline{\text { Parental characteristics }}$}

Parents were asked to indicate their own postpartum weight and height, which we used to calculate their BMI. A BMI above $25 \mathrm{~kg} / \mathrm{m}^{2}$ was regarded as overweight. Additionally, maternal country of birth, age at delivery of the child, highest completed education and the number of hours that each of the parents worked per week were assessed. Maternal education was categorised using median split, into lower (including elementary school, lower general secondary education, and lower 
and intermediate vocational education) and higher educational level (including higher general secondary education, higher vocational education, college-prep and university).

\section{Data analysis}

All statistical analyses were conducted using SPPS 15.0 (SPSS Inc., Chicago, Illinois, U.S.A.). First, the distribution of demographic background variables was explored. In all analyses with BMI z-scores, overweight or change in BMI or overweight as dependent variables, children who were underweight (BMI z-score $\leq$ $5^{\text {th }}$ percentile; (Barlow, 2007) were excluded, because an increase in BMI would be desirable for these children. This regarded 131 children (5.7\%) at age 1, and 118 children $(5.4 \%)$ at age 2 . The influence of child-care use on BMI z-scores and overweight was tested using linear and binary logistic regression analyses, respectively. In a separate step, the various background characteristics of the children and parents (number of hours of employment and overweight of mother and of father; maternal educational level, age and country of birth; parental alternative lifestyle; child's birth weight; breast-feeding duration) were included and backward deleted in order of their significance. Dependent variables were 1) BMI z-score at 1 and 2 years of age; 2) the change in BMI z-score between ages 1 and 2 years; 3 ) overweight vs. non-overweight at age 1 and 2 years; and 4) change from normal weight to overweight vs. remaining normal weight between 1 and 2 years of age. The analyses with weight status change as dependent variable (4) included only children at risk of becoming overweight (children who were already overweight at age 1 were excluded, $\mathrm{N}=347$ ).

Additional linear and logistic regression analyses were performed within the group of children who attended child-care, comparing the influence of intensive versus limited child-care use on 1) BMI z-score; 2) BMI z-score change; 3) overweight; and 4) weight status change. In order to adjust for the above-mentioned covariates, they were included in the analyses in a separate step and backward deleted. In all analyses, p-values $<.05$ were considered statistically significant, and $\mathrm{p}$-values $<.10$ were interpreted as indicating a trend.

To examine predictors of child-care use, the individual association between parental background variables and child-care use (yes/no) at 7 months, 1 year and 2 years was assessed using various univariate binary logistic regression analyses. In addition, all parental background variables were simultaneously included in multivariate backward binary logistic regression analyses, to examine the influence of each of the variables on child-care use, adjusted for the influence of the other variables. Non-significant independent variables were deleted from these analyses in order of their significance, starting with the least significant. 


\section{Results}

Questionnaires regarding 2396 children were returned at all three ages. Background information regarding these children and their parents can be found in Table 6.1. About a third of the children attended child-care at the various ages, for an average of about 20 hours per week. A total of 628 children attended child-care at all three ages. Around $15 \%$ of the children were overweight at ages 1 and 2 years.

Table 6.1: Descriptive information of background characteristics $(\mathrm{N}=2396)$

\begin{tabular}{|c|c|c|c|c|}
\hline & & & Mean \pm SD & Prevalence (\%) \\
\hline \multirow[t]{10}{*}{ Child } & Gender & & & male: $51.5 \%$ \\
\hline & & & & female: $48.5 \%$ \\
\hline & $\begin{array}{l}\text { Birth weight } \\
\text { (grams) }\end{array}$ & & $3526 \pm 505$ & \\
\hline & Child-care attendance & $7 \mathrm{~m}$ & $20.0 \pm 7.3$ & $31.6 \%$ \\
\hline & (hours per week ${ }^{a}$ and & $1 \mathrm{y}$ & $20.1 \pm 7.4$ & $33.2 \%$ \\
\hline & yes vs. no) & $2 y$ & $19.5 \pm 8.0$ & $38.6 \%$ \\
\hline & BMI z-score & $1 \mathrm{y}$ & $-.02 \pm 1.03$ & \\
\hline & & $2 y$ & $-.04 \pm 1.05$ & \\
\hline & Overweight & $1 \mathrm{y}$ & & $15.2 \%$ \\
\hline & (BMI $z-$ score $\geq 85^{\text {th }}$ percentile) & $2 \mathrm{y}$ & & $14.4 \%$ \\
\hline \multirow[t]{8}{*}{ Maternal } & $\begin{array}{l}\text { Employment } \\
\text { (hours per week) }\end{array}$ & & $18.6 \pm 12.0$ & \\
\hline & Educational level $^{b}$ & & & high: $62.1 \%$ \\
\hline & & & & low: $37.9 \%$ \\
\hline & $\begin{array}{l}\text { Age at birth of child } \\
\text { (years) }\end{array}$ & & $32.1 \pm 3.7$ & \\
\hline & Country of birth & & & $\begin{array}{l}\text { NL: } 96.5 \% \\
\text { other: } 3.5 \%\end{array}$ \\
\hline & Alternative lifestyle ${ }^{c}$ & & & $19.1 \%$ \\
\hline & (yes vs. no) & & & \\
\hline & $\begin{array}{l}\text { Overweight } \\
\left(B M I>25 \mathrm{~kg} / \mathrm{m}^{2}\right)\end{array}$ & & & $31.6 \%$ \\
\hline \multirow[t]{2}{*}{ Paternal } & $\begin{array}{l}\text { Employment } \\
\text { (hours per week) }\end{array}$ & & $39.7 \pm 10.1$ & \\
\hline & $\begin{array}{l}\text { Overweight } \\
\left(B M I>25 \mathrm{~kg} / \mathrm{m}^{2}\right)\end{array}$ & & & $45.9 \%$ \\
\hline \multicolumn{5}{|c|}{$\begin{array}{l}{ }^{a} \text { Hours per week child-care attendance reflects the average within the group that attends child-care. } \\
{ }^{b} \text { High is }>\text { median, low is } \leq \text { median. }\end{array}$} \\
\hline \multicolumn{5}{|c|}{${ }^{c}$ Recruited through alternative channels, vs. through conventional channels. } \\
\hline
\end{tabular}

Table 6.2 shows the association between child-care attendance and 1) BMI z-scores at 1 and 2 years; and 2) change in BMI z-scores between these ages. Child-care attendance was positively associated with BMI z-score at 2 years. The association with BMI z-score at 1 year was not significant when adjusting for covariates. Child- 
care attendance at age 1 was further positively associated with the change in BMI zscore between 1 and 2 years of age: children attending child-care showed an increase in BMI z-score, while children not attending child-care showed a decrease.

Within the group of children using child-care at each age, there were no statistically significant differences in the influence on BMI z-scores between limited ( $\leq 16$ hours a week) and intensive ( $>16$ hours a week) child-care use after adjustment for covariates. The unadjusted analyses did show a significant difference between limited and intensive use at age 2 with regard to the association with BMI $\mathrm{z}$-score at the same age (mean BMI z-scores for limited vs. intensive child-care use: .07 vs. .21, $\mathrm{p}<.05$ ). Mean BMI $\mathrm{z}$-score was positive in both groups because underweight children were excluded.

Table 6.2: Association between child-care use (no/yes) and BMI $z$-scores at ages $1(\mathrm{~N}=2156)$ and 2 years ( $\mathrm{N}=2086)$, and change in BMI $\mathrm{z}$-score between 1 and 2 years $(\mathrm{N}=1922)$, resulting from univariate (unadjusted) and backward multivariate (adjusted for covariates) linear regression analyses

\begin{tabular}{|c|c|c|c|c|}
\hline \multirow[t]{2}{*}{ Child-care } & No $(0)$ & Yes (1) & \multirow{2}{*}{ Unadjusted B } & \multirow{2}{*}{ Adjusted B } \\
\hline & \multicolumn{2}{|c|}{ Mean BMI z-score } & & \\
\hline & \multicolumn{4}{|c|}{ BMI z-score 1 year } \\
\hline 7 months & .07 & .18 & $.11^{* *}$ & .02 \\
\hline \multirow[t]{2}{*}{1 year } & .08 & .17 & $.09 *$ & -.02 \\
\hline & \multicolumn{4}{|c|}{ BMI z-score 2 years } \\
\hline 7 months & .04 & .17 & $.12^{* *}$ & $.08^{\dagger}$ \\
\hline 1 year & .04 & .17 & $.13^{* *}$ & $.12 *$ \\
\hline \multirow[t]{2}{*}{2 years } & .04 & .15 & $.11^{* *}$ & $.12 *$ \\
\hline & \multicolumn{4}{|c|}{ BMI z-score change 1-2 years } \\
\hline 7 months & -.02 & .03 & .05 & .02 \\
\hline 1 year & -.04 & .06 & $.10^{*}$ & $.10^{*}$ \\
\hline
\end{tabular}

\section{Influence of child-care use on overweight development}

Table 6.3 shows the association between child-care attendance and 3) overweight vs. normal weight at 1 and 2 years; and 4) change to overweight vs. remaining normal weight between these ages. Although the percentage of overweight children was consistently higher in the group attending child-care (all OR's $>1$ ), these differences were not significant. However, after adjustment for significant covariates, child-care attendance at 7 months significantly increased the odds of being overweight at age 1 (Odds Ratio $(\mathrm{OR})=1.32$, Confidence Interval $(\mathrm{CI})=1.04$ 1.69). There was a trend $(\mathrm{p}<.10)$ for children in child-care to have higher odds of becoming overweight between the ages of 1 and 2 years.

At age $1,15.9 \%$ of the children making limited use of child-care $(\leq 16$ hours a week) at 7 months were overweight, while $21.8 \%$ were overweight in the 
group that used child-care intensively ( $>16$ hours a week). This difference was significant $(\mathrm{p}<.05 ; \mathrm{OR}=1.81, \mathrm{CI}=1.12-2.92)$.

Table 6.3: Association between child-care use (no/yes) and overweight (BMI z-score $\geq 85^{\text {th }}$ percentile) at ages $1(\mathrm{~N}=2156)$ and 2 years $(\mathrm{N}=2086)$, and change in weight status between 1 and 2 years $(\mathrm{N}=1576)$, resulting from univariate (unadjusted) and backward multivariate (adjusted for covariates) binary logistic regression analyses

\begin{tabular}{|c|c|c|c|c|}
\hline Child-care & $\begin{array}{r}\text { No (0) } \\
\text { Percen }\end{array}$ & $\begin{array}{l}\text { Yes (1) } \\
\text { rweight }\end{array}$ & $\begin{array}{c}\text { Unadjusted OR } \\
(95 \% \mathrm{CI})\end{array}$ & $\begin{array}{c}\text { Adjusted OR } \\
(95 \% \mathrm{CI})\end{array}$ \\
\hline & \multicolumn{4}{|c|}{ Overweight 1 year } \\
\hline 7 months & $14.8 \%$ & $18.8 \%$ & $1.06(.79-1.42)$ & $1.32 *(1.04-1.69)$ \\
\hline \multirow[t]{2}{*}{1 year } & $15.2 \%$ & $18.1 \%$ & $1.05(.79-1.40)$ & $1.23^{\dagger}(.97-1.57)$ \\
\hline & \multicolumn{4}{|c|}{ Overweight 2 years } \\
\hline 7 months & $14.3 \%$ & $16.8 \%$ & $1.07(.79-1.44)$ & $1.18(.89-1.57)$ \\
\hline 1 year & $14.4 \%$ & $16.3 \%$ & $1.09(.81-1.46)$ & $1.17(.88-1.55)$ \\
\hline \multirow[t]{2}{*}{2 years } & $14.1 \%$ & $16.5 \%$ & $1.17(.88-1.57)$ & $1.19(.93-1.53)$ \\
\hline & \multicolumn{4}{|c|}{ Changed from normal weight to overweight $1-2$ years } \\
\hline 7 months & $8.3 \%$ & $11.0 \%$ & $1.25(.84-1.88)$ & $1.36^{\dagger}(.95-1.94)$ \\
\hline 1 year & $8.2 \%$ & $11.0 \%$ & $1.23(.83-1.85)$ & $1.37^{\dagger}(.96-1.95)$ \\
\hline \multicolumn{5}{|c|}{$\begin{array}{l}p<.10 ; * p<.05 \text {. } \\
\text { OR=Odds Ratio; CI=Confidence interval. The numbers deviate from total sample size due to exclusion } \\
\text { of underweight children (BMI z-score } \leq 5^{\text {th }} \text { percentile). The number for weight status change further } \\
\text { deviates from total sample size because only children at risk for becoming overweight (i.e., normal } \\
\text { weight children) were included. }\end{array}$} \\
\hline
\end{tabular}

\section{Associations between parental background characteristics and child-care use}

Child-care use was associated with various parental background characteristics (see Table 6.4). The average number of hours that the mother worked per week was positively and significantly associated with child-care use at all ages: for each additional hour the mother worked, the probability of child-care use increased by 6$8 \%$. The association between child-care use and the father's work was positive, but much weaker and only became significant after adjustment for covariates at 7 months and 1 year. Children of highly educated mothers were more than twice as likely to be in child-care than children of mothers with a lower educational level. Children of older mothers were also more likely to attend child-care. Having an alternative lifestyle was associated with less child-care use at the age of 7 months, but the association was not significant at older ages. The country of birth of the mother was unrelated to child-care use. 


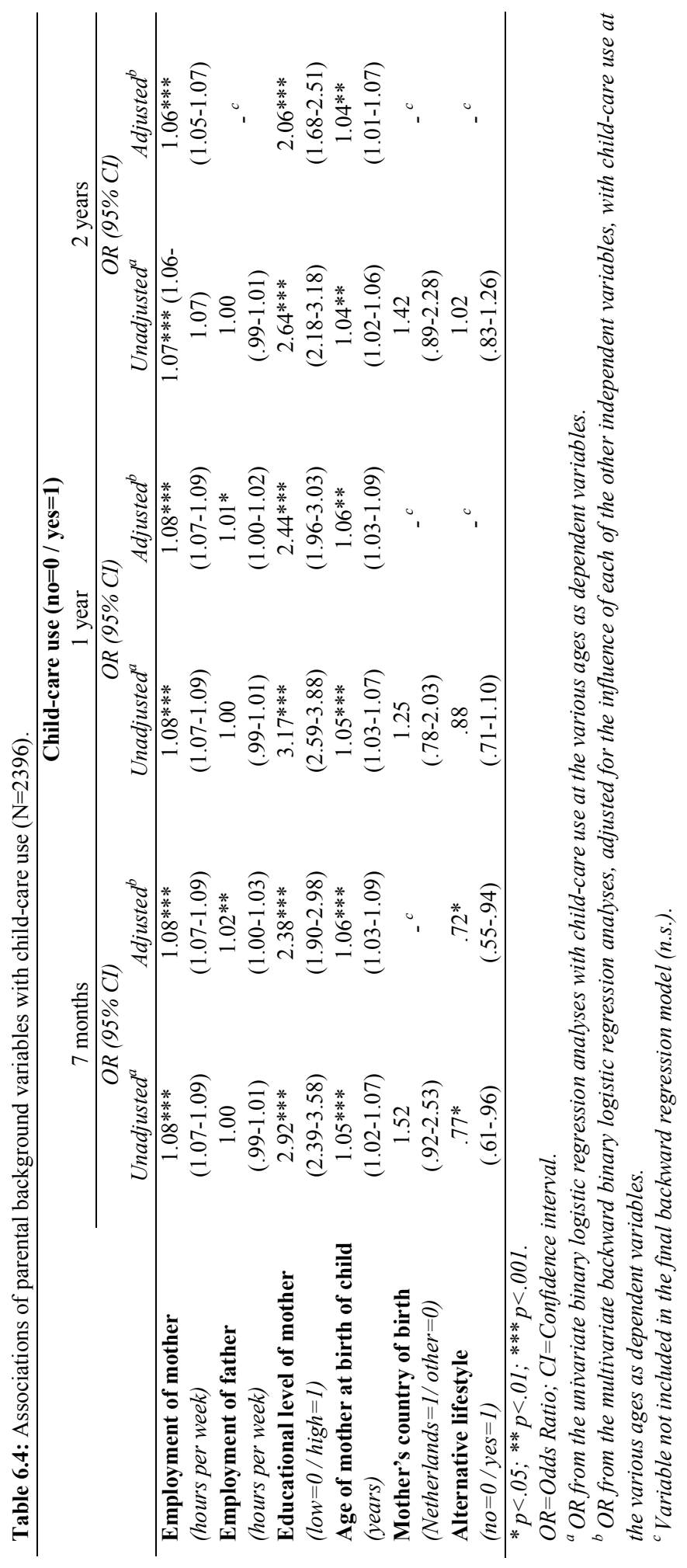




\section{Discussion}

The current study showed that child-care attendance at the child's age of 1 year and 2 years was positively associated with BMI z-scores at 2 years, and a greater increase in BMI z-score between these ages. In addition, to a limited extent, childcare use increased the odds of being overweight at age 1, and developing overweight between the ages of 1 and 2 years. These findings are in line with those of some previous studies (Benjamin et al., 2009; Kim \& Peterson, 2008; Maher et al., 2008). However, other studies have reported protective effects of child-care use against overweight or obesity (Frisvold \& Lumeng, 2007; Lumeng et al., 2005), or found no effect (Kimbro et al., 2007; Rapp et al., 2005). These conflicting findings might be explained by differences between the study populations. For instance, several previous studies included low educated or low income populations (Frisvold \& Lumeng, 2007; Zaslow et al., 1998). In our current sample, the majority of the mothers $(62.1 \%)$ had a high educational level. Since educational level has been shown to be positively associated with the quality of mothers' parenting practices (Brown et al., 2008; Gubbels et al., 2009), child-care use could possibly be beneficial for children of low educated mothers, but might have no protective effect for children of highly educated mothers. Another difference between the study populations regards the age of the children. All studies of child-care use in young children $(<3$ years), including the current study, found a positive relationship between child-care use and overweight (Benjamin et al., 2009; Kim \& Peterson, 2008). In contrast, the studies among older children ( $\geq 3$ years) showed conflicting results (Frisvold \& Lumeng, 2007; Kimbro et al., 2007; Lumeng et al., 2005; Maher et al., 2008; Rapp et al., 2005; Wronka \& Pawlinska-Chmara, 2007). Possibly, child-care is specifically unfavourable for young children, but not so much for older children. Moreover, the various studies differ regarding their study designs and methods (e.g., some studies are cross-sectional while others are longitudinal), which might explain the conflicting findings. An alternative explanation for the conflicting findings of different studies might be that the child-care centres attended in the various studies differed in terms of their 'obesogenicity'. Previous research has shown that various aspects of the child-care environment influence dietary intake and physical activity (Bower et al., 2008; Gubbels et al., 2010; Hughes et al., 2007; Pate et al., 2008). Unfortunately, no information regarding the quality of the childcare centres was available in the current or previous studies. Additionally, differences in the overall child-care systems in the different studies and countries (e.g., different opening hours, different policies), might have influenced the findings.

In addition to child-care related factors being responsible for an association between child-care use and BMI, the relationship found between the two might also be confounded by factors associated with both child-care use and BMI. For instance, Kim and Peterson (2008) found that child-care by a relative was associated 
with both unfavourable infant feeding practices, such as lower breast-feeding rates, and more weight gain in the first 9 months of life. Although the association between child-care use and BMI remained significant after adjustment for factors like breastfeeding duration in the current study, and secondary analyses did not reveal a significant association between child-care and breast-feeding, other factors that were not assessed might also have confounded this relationship. An example of such a factor is infectious diseases. Children that attend child-care are more likely to be infected with several communicable diseases (Bradley \& Vandell, 2007). These diseases and the medication taken for them might influence children's weight and/or height.

Child-care attendance showed a weaker association with BMI at 1 year than with BMI at 2 years, and the association at age 1 even disappeared after adjustment for covariates in the analyses. The analyses with overweight as a dependent variable showed the opposite outcome: there was a significant association between child-care use and overweight at age 1, but not at age 2 . It is unclear why this is the case, but the differences in eating and activity patterns between 1-year-olds and 2-year-olds could play a role. For instance, 1-year-old infants drink more milk than 2-year-olds (World Health Organization, 2009), and spend more hours during the day sleeping (Davis, Parker, \& Montgomery, 2004). The child-care environment may have differential influences on the different dietary and physical activity behaviours at different ages, leading to differences in the association between child-care use and weight status.

The only difference between intensive ( $>16$ hours per week) and limited use ( $\leq 16$ hours) was that intensive child-care at 7 months led to more overweight at age 1 . The absence of other significant differences indicates that child-care use in general is mostly associated with a higher BMI, regardless of the amount of time spent in child-care. In line with the current findings, previous studies reported more intensive child-care use to be associated with a higher BMI, compared to limited use (Maher et al., 2008). However, intensive child-care has also been reported to be associated with either a weaker (Lumeng et al., 2005) or a stronger (Frisvold \& Lumeng, 2007) negative association with BMI, compared to limited use.

In addition to examining the influence of child-care use on BMI and overweight, the current study also explored correlates of child-care use itself. Various parental characteristics were significantly related to child-care, including maternal age and parental working hours. In line with previous research (Huston et al., 2002; Wronka \& Pawlinska-Chmara, 2007; Zaslow et al., 1998), children were found to be more than twice as likely to attend child-care if they had a mother who had a high educational level, and were also significantly more likely to attend childcare for each additional hour their mother worked. Although educational level and employment hours could both be seen as indicators of socio-economic status, their associations with child-care use remained significant (although somewhat weakened) after adjustment for each other and other covariates. Educational level 
and employment are related to parental income (not assessed in the current study), which could partly explain their association with child-care use, as parental income has previously been linked to child-care use (Lumeng et al., 2005). Costs of childcare are relatively high, and parents with a higher income are more likely to be able to afford it. In contrast to maternal working hours, father's working hours were found to have only minor influence on child-care use at 7 months and 1 year. This difference could be explained by the fact that fathers are traditionally less involved in child rearing and often work full-time (39.7 hours per week on average in the current sample). Children who do not attend child-care are often cared for by their mothers at home, which explains the positive relationship between maternal employment and child-care attendance.

Some limitations should be taken into account in interpreting the results. A limitation of the current study is that it only examined formal child-care arrangements, while some previous studies have shown the differential influence of different types of child-care (Benjamin et al., 2009; Kim \& Peterson, 2008; Kimbro et al., 2007; Maher et al., 2008). Furthermore, all data, including weight, height and child-care attendance, were reported by parents, and could therefore be biased. However, the questionnaires were completed shortly after visits to infant welfare centres, and parents were asked to take down the results of the anthropometric measurements made there. Although child anthropometric measures are probably more reliable when assessed by health care professionals compared to assessment by parents (Scholtens et al., 2007), using this approach also introduces an additional error source: parents could makes mistakes when copying these data. BMI distribution in the study population was, however, similar to that in the reference population (Fredriks et al., 2000). A strong point of the current study is that data were gathered longitudinally, so recall bias and other weaknesses of retrospective research are less probable in the current study. However, child-care use was only assessed at the time of the questionnaire, and child-care attendance between questionnaires was not asked.

Various authors have indicated the important role that child-care could play in the prevention of overweight and obesity (see e.g., Story et al., 2006). The current study contributes to the still limited evidence base supporting this proposition. Previous research has identified modifiable factors in the child-care environment that influence energy balance-related behaviours, such as staff behaviour and availability of facilities (Bower et al., 2008; Gubbels et al., 2010; Hughes et al., 2007; Pate et al., 2008). When combining this knowledge with the fact that children are spending increasing amounts of time in child-care (Blackburn, 2006; Eurostat, 2008), it would appear that interventions focusing on these factors could contribute to counteracting the childhood overweight and obesity epidemic. Although more research will be needed to specify the content of such interventions, recent interventions educating parents, staff and children showed promising results (Jouret et al., 2009). 



\section{Chapter 7: Child-care environment and dietary intake of 2- and 3-year-old children}

This chapter has been published as: Gubbels, J.S., Kremers, S.P.J., Stafleu, A., Dagnelie, P.C., de Vries, N.K. \& Thijs, C. (2010). Child-care environment and dietary intake of 2- and 3-year-old children. Journal of Human Nutrition and Dietetics, 23(1), 97-101. 


\begin{abstract}
Background: Previous research has shown that children at child-care do not comply with dietary intake recommendations (i.e., either exceeding or not meeting recommendations), which may be attributable to specific features of the child-care environment. The present study explored the relationship between the social and physical child-care (day-care) environment and dietary intake of 2- and 3-year-olds in Dutch child-care centres.

Methods: Dietary intake of 135 children, aged 2 and 3 years, who were in child-care was assessed by observing randomly selected children at three meals (morning snack, lunch and afternoon snack), to determine dietary intake (i.e., saturated fat, dietary fibre and energy intake). The environment was observed using the Environment and Policy Assessment and Observation checklist, a structured instrument assessing the physical and social environment.

Results: Children consumed a mean of $486 \mathrm{~kJ}$ (116 kcal) during morning snack, $2043 \mathrm{~kJ}$ (488 kcal) during lunch and $708 \mathrm{~kJ}$ (169 kcal) during afternoon snack. There were some gender and age differences in dietary intake. Several environmental factors (e.g., serving style and staff's model dietary behaviour) were significantly associated with the children's dietary intake.

Conclusions: Overall, energy intake was in the upper range of recommended intake for children in child-care. The associations of several environmental factors with dietary intake stress the importance of the child-care environment for children's dietary behaviour. Intervening in this setting could possibly contribute to comprehensive prevention of childhood obesity.
\end{abstract}




\section{Introduction}

The childhood prevalence of being overweight is increasing world-wide (World Health Organization, 2006), with dietary intake playing a crucial role (Rennie et al., 2005). Moreover, eating habits are often established at a young age (Savage et al., 2007) and maintained throughout life (Kelder et al., 1994).

Child-care attendance is increasing (Portegijs, Hermans, \& Lalta, 2006), implying a potentially large influence of this setting on children's development. It has been recommended that a child who spends a full day (i.e., $8 \mathrm{~h}$ or more) in child-care should consume one-half to two-thirds of his or her daily dietary intake in child-care (American Dietetic Association, 1994). Various studies in the USA have shown that children in child-care do not meet dietary intake recommendations (Ball, Benjamin, \& Ward, 2008; Bollella et al., 1999; Briley, Jastrow, Vickers, \& Roberts-Gray, 1999; Padget \& Briley, 2005). Specific features of the child-care environment may underlie such findings. Hughes and colleagues (2007), for example, found that indulgent and authoritative feeding styles of child-care staff were positively associated with vegetable and dairy consumption (Hughes et al., 2007). In addition, just as parents can apply certain parenting practices to influence their child's dietary intake (e.g., Gubbels et al., 2009), child-care staff also apply specific supervision practices.

The present study explored dietary intakes (i.e., saturated fat, dietary fibre and energy intake) and the presence of various environmental factors (i.e., physical environment, food serving style, supervision practices and staff behaviour) in Dutch child-care centres. Observations were executed during the three meals that children in the Netherlands usually receive in child-care: a morning snack, lunch and an afternoon snack. In addition, the associations between these variables were explored.

\section{Materials and Methods}

\section{Participants and Setting}

The study complied with the Dutch 'Medical Research Involving Humans Act'. All nine child-care centres of the coordinating organisation MIK (Maastricht, the Netherlands) were contacted and agreed to participate. MIK gave consent for the study. Parents were informed in a letter; none refused participation.

\section{Assessment of dietary intake and child-care environment}

Observations took place in May and June 2008. Children were observed during their morning snack and lunch on 1 day, and during their afternoon snack on a second day, in a non-consecutive week. At each meal, five children were randomly 
selected, resulting in a total of 135 observations (nine centres, three meals per centre, five children per meal). The intake and environment were observed for each child separately, and recorded simultaneously by two observers, who did not interact with each other and were trained in the use of the observation protocol.

Dietary intake was estimated in standard food units (e.g., cups, pieces), and recorded on a structured form developed for this study, listing common food items and their type (e.g., whether milk was skimmed, semi-skimmed or whole). Only actually consumed foods were recorded, not leftovers and spoiled foods.

The child-care environment was assessed using items from the Environment and Policy Assessment and Observation instrument (Ball et al., 2005), which were selected, translated and adjusted to fit the Dutch child-care system. Table 7.1 lists the environmental factors assessed. In addition, children's gender and age were assessed by asking staff after the observations.

\section{Statistical Analyses}

Dietary intake was calculated as the mean of the observations by the two observers, after converting standard food units into grams, and subsequently into intake of energy, saturated fat and dietary fibre (Stichting Nederlands Voedingsstoffenbestand, 2006). Cohen's kappa was .84 ( $\mathrm{SD}=.15)$, intra-class correlation was .91 $(\mathrm{SD}=.14)$, both indicating good inter-observer agreement (Field, 2005). Total mean intake was calculated as the sum of the mean intakes at each meal per child-care centre. Associations between intake and environment were explored using Point biserial correlations. Results with $\mathrm{p}<.05$ were considered statistically significant.

\section{Results}

In total, 135 children were observed. Five children (during lunch) were excluded from analyses, because they were not simultaneously observed by both observers. Forty-three percent of the children were 2 years old, the remaining $57 \%$ were 3 years old. Fifty-four percent were male.

There were an mean (SD) of 11.5 (2.3) children and $2.2(.5)$ staff members (all female) present at the meals. In most cases, staff ate together with the children, and they mostly ate the same food (Table 7.1). More than half of the children were stimulated to eat more than they wanted to (e.g., 'Finish your sandwich!'), for an average of 1.8 times, but ranging up to 10 times per child during one meal. Children were encouraged to try an alternative option at most once per meal (e.g., 'We're out of apples, don't you want to try this tasty pear?'), whereas punishment by (temporarily) taking food away happened twice per meal at most. 


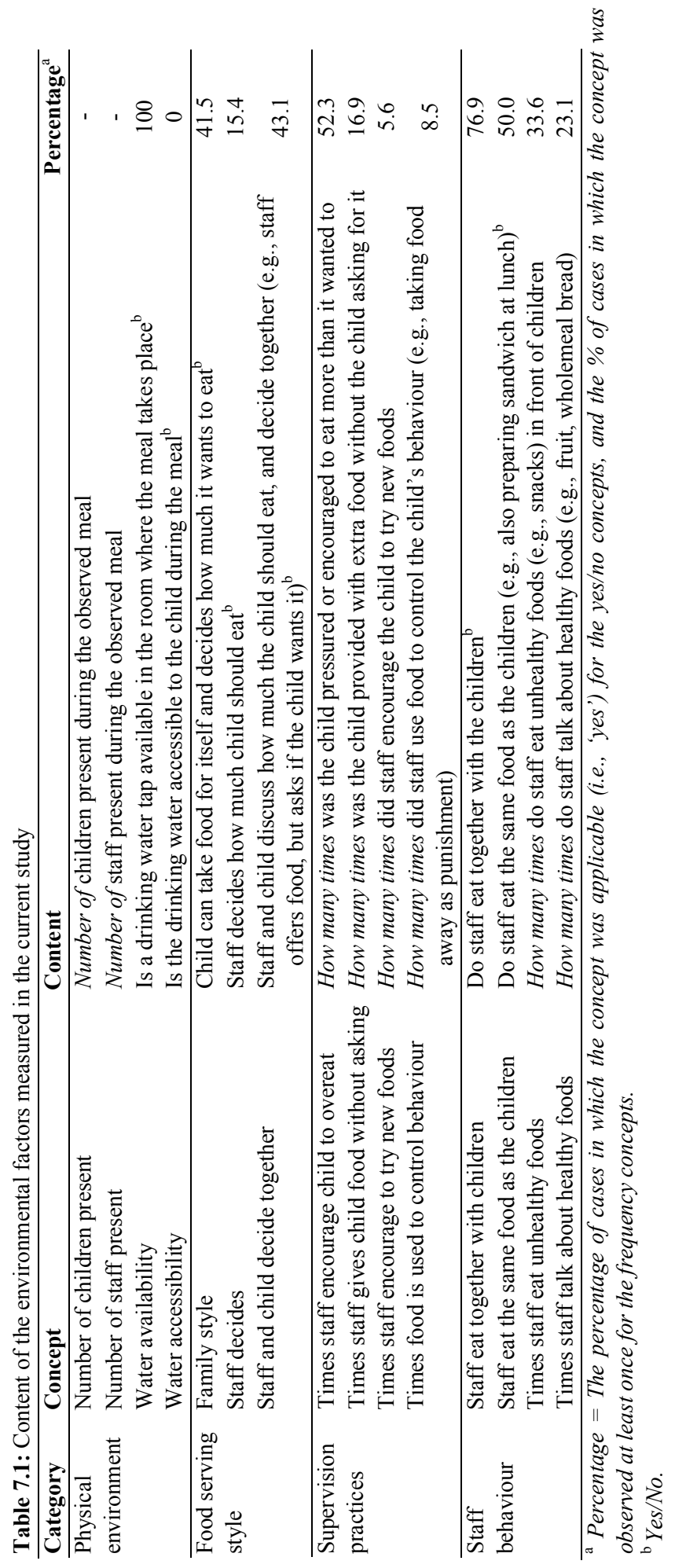




\section{Children's dietary intake}

The children generally consumed fruit in the morning, sandwiches at lunch and snacks (e.g., biscuits) during the afternoon. Total mean energy intake during a full day at child-care was $3237 \mathrm{~kJ}$ (773 kcal; Table 7.2). Three-year-olds had higher saturated fat intakes at lunch than 2-year-olds (8.6 vs. $6.7 \mathrm{~g} ; \mathrm{p}=.04)$; boys consumed more energy than girls during the afternoon (770 vs. $628 \mathrm{~kJ}$, i.e., 184 vs. $150 \mathrm{kcal}$; $\mathrm{p}=.04)$.

Table 7.2: Intake of 2- and 3-year-old children in child-care centres

\begin{tabular}{lccc}
\hline & \multicolumn{3}{c}{ Dietary intake mean $($ SD) } \\
\cline { 2 - 4 } & Energy $(\mathrm{kJ})$ & Saturated fat $($ grams $)$ & Dietary fibre $($ gram $)$ \\
\hline Morning snack $(N=45)$ & $486(239)$ & $.2(.7)$ & $1.9(.8)$ \\
Lunch $(N=40)$ & $2043(615)$ & $7.8(2.8)$ & $5.9(2.1)$ \\
Afternoon snack $(N=45)$ & $708(234)$ & $1.0(1.1)$ & $.8(.9)$ \\
\hline
\end{tabular}

Environmental factors and background characteristics

Staff were less inclined to encourage girls to try new things $(r=-.20, p<.05)$, and more often used food to control girls' behaviour $(\mathrm{r}=.19, \mathrm{p}<.05)$. Staff more often talked about healthy foods to older children $(\mathrm{r}=.18, \mathrm{p}<.05)$. Staff at larger centres less often used food to control behaviour $(\mathrm{r}=-.43, \mathrm{p}<.01)$, but more often ate unhealthy foods in front of the children $(\mathrm{r}=.47, \mathrm{p}<.01)$. When more children were present, staff were more likely to decide how much a child should eat $(\mathrm{r}=.49$, $\mathrm{p}<.01$ ), instead of deciding together with the child or applying a family style ( $\mathrm{r}=$ $.18, \mathrm{p}<.05$ for both variables), and were also more likely to eat the same food as the children $(\mathrm{r}=.19, \mathrm{p}<.05)$.

\section{Dietary intake and environmental factors}

A family serving style was associated with higher saturated fat and dietary fibre intake (Table 7.3). Children ate more when staff ate together with them, regardless whether eating the same or unhealthy foods. Talking about healthy foods was associated with higher fibre intake. Encouragement to overeat was associated with lower intakes during lunch. Giving a child food without asking was associated with higher dietary fibre intake. Using food to control behaviour was associated with lower fibre intake. 


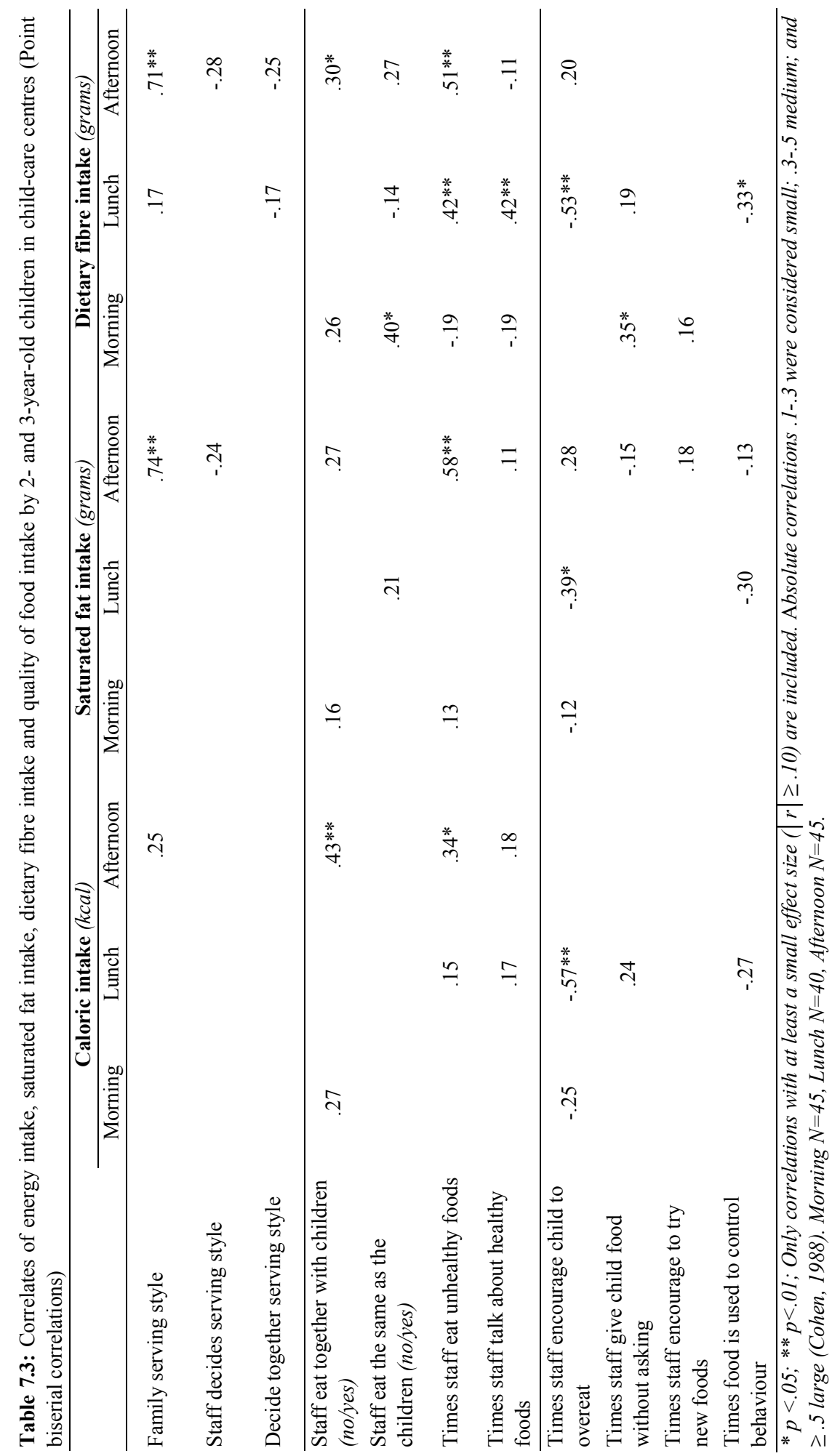




\section{Discussion}

Total mean energy intake in the current sample $(3237 \mathrm{~kJ}, 773 \mathrm{kcal})$ was in the upper range of recommended intake $(2512-3349 \mathrm{~kJ}$, i.e., 600-800 kcal); saturated fat and fibre intake complied with recommendations (American Dietetic Association, 1994; Voedingscentrum, 2007). Note that, these mean intakes merely provide an indication of total intake: they reflect a summed total of the means per meal, incorporating intakes of different children, though from the same centre.

As far we are aware, this is the first study to examine dietary intake and environment at European child-care centres, and the first to describe associations between distinct environmental characteristics and intake. The results obtained extend those found by Hughes and colleagues (2007), who examined the association between feeding style and dietary intake. Note, however, that the current research was explorative. The findings need to be confirmed in larger samples, allowing correction for potential bias introduced by the multilevel design. Strong points were the validated observation protocol (Ball et al., 2005) and high inter-observer reliability.

The family serving style appeared to induce children to eat more, regardless of the food served, which is interesting. Previous research indicated that a family style, in which children can respond to satiation cues and are less likely to overeat, is best for the child's eating habits development (e.g., Fisher, Rolls, \& Birch, 2003; Johnson \& Birch, 1994). The children in the present study might have been too young to regulate their own intake. Further research should examine at what age the family style would be desirable. Encouragement to overeat was associated with lower intakes, probably indicating that picky or slow eaters were encouraged to eat more.

Staff appeared to function as role models, with higher intake by children when staff ate together with them. This is inconsistent with earlier findings showing that teacher modelling was unrelated to preschool children's intake. Enthusiastic modelling (i.e., promoting food after eating it), however, was reported to be effective (Hendy \& Raudenbush, 2000), in line with the current findings.

The present study underlines the importance of child-care in determining children's dietary intake. However, more research is needed to examine the mechanisms underlying the association between child-care environment and children's dietary intake. In addition, cultural differences warrant caution when generalising results of US studies to the European context. In summary, intervening in the child-care setting, with special attention to staff behaviour, could help children to establish healthier eating habits and prevent them becoming overweight. 


\section{Chapter 8: Interaction between environ- mental factors determining physical activity of children at child-care}

This chapter has been accepted for publication as: Gubbels, J.S., Kremers, S.P.J., van Kann, D.H.H., Stafleu, A., Candel, M.J., Dagnelie, P.C., Thijs, C. \& de Vries, N.K. (In Press). Interaction between environmental factors determining physical activity of children at child-care. Health Psychology. 


\begin{abstract}
Objective: This study investigated the association between the child-care environment and physical activity of 2- and 3-year-olds. Based on an ecological view of environmental influences on health behaviour, we hypothesized that the social and physical environment, as well as child characteristics (age and gender), would show independent and interactive effects on children's physical activity intensity.

Design: Observations of physical activity intensity were performed among 175 children at 9 Dutch child-care centres. Aspects of the child-care environment were assessed using the validated EPAO instrument. Multilevel linear regression analyses examined the association of environment and child characteristics with children's activity intensity. Moderation was tested by including interaction terms in the analyses, with subsequent post-hoc analyses for significant interaction terms.

Main Outcome Measure: Observed child physical activity intensity, measured with the OSRAC-P.

Results: A large proportion of the observed activities were classified as sedentary, while far fewer observations were classified as moderate or vigorous. Activity opportunities in the physical environment (assessed using EPAO) and prompts by staff and peers were significantly and positively related to physical activity intensity, while group size was negatively related to activity intensity. The influence of the physical environment was moderated by social environment (peer group size), while the social environment in turn interacted with child characteristics (age and gender) in determining activity intensity.

Conclusion: Our findings are in line with the ecological perspective regarding environmental influences on behaviour, and stress the importance of incorporating the child-care environment in efforts to prevent childhood overweight and obesity.
\end{abstract}




\section{Introduction}

The prevalence of childhood overweight and obesity is increasing worldwide (World Health Organization, 2006), and weight status often tracks from childhood into adulthood (Singh et al., 2008). Low levels of physical activity are among the most important causes of childhood overweight (Rennie et al., 2005). Since physical activity habits are formed at a young age (Reilly et al., 2004), and once established, are often maintained in later life (Kelder et al., 1994), targeting physical activity in early childhood is essential in order to prevent obesity.

In Europe, over half of the toddlers attend some form of child-care or education facilities (Blackburn, 2006). This setting offers a potential opportunity for environmental interventions to promote physical activity among young children (McWilliams et al., 2009; Story et al., 2006). Such interventions can only be systematically designed after the most important environmental determinants have been identified (Brug, Oenema, \& Ferreira, 2005).

A recent review of correlates of preschool children's physical activity showed that the preschool a child attends is significantly associated with the child's physical activity (Hinkley et al., 2008). Several authors have called for additional research to identify the specific characteristics of preschools that explain variance in physical activity levels between preschools and child-care centres (Brown et al., 2009; Pate et al., 2008). Specific characteristics of the physical child-care environment linked to increased physical activity include the availability of play equipment and activity opportunities (Bower et al., 2008; Hannon \& Brown, 2008; Zask, van Beurden, Barnett, Brooks, \& Dietrich, 2001), a 'natural' environment (Boldemann et al., 2006), shorter recess duration (Cardon et al., 2008; Dowda, Pate, Trost, Almeida, \& Sirard, 2004), colour markings at playgrounds (Ridgers, Stratton, Fairclough, \& Twisk, 2007b; Stratton, 2000), smaller school size (Zask et al., 2001) and higher overall classroom quality (Dowda et al., 2004). However, the importance of moving beyond an isolated view on physical environmental factors in child-care was recently indicated by Cardon et al. (Cardon, Labarque, Smits, \& Bourdeaudhuij, 2009), who showed that interventions altering the physical environment at child-care alone were not effective in increasing children's physical activity. Social environment factors previously found to be associated with increased physical activity include smaller group size (Brown et al., 2009; Cardon et al., 2008), child-initiated instead of staff-initiated play (Brown et al., 2009), and a higher educational level of staff members (Dowda et al., 2004).

It has been theorized that the influence of the environment on physical activity behaviour can be moderated by several factors, including demographic characteristics such as age and gender (Anderson, Hughes, \& Fuemmeler, 2009; Kremers et al., 2006; Kremers et al., 2008). In fact, age has been found to moderate the effect of the physical child-care environment on physical activity, with playground markings having more effect on younger children compared to older 
children (Ridgers et al., 2007a). In addition, indications were found that the social preschool environment has a stronger influence on girls than on boys (Cardon et al., 2008). Variables other than demographic characteristics could also moderate the influence of the child-care environment on children's physical activity. The ecological 'systems' view of environmental influences on behaviour in general (Friedman \& Wachs, 1999; Wachs, 1992) and physical activity in particular (Spence \& Lee, 2003) assumes that within the so-called micro system dimension, physical environmental factors (i.e., features of the immediate behavioural setting) interact with the actors that operate within this setting (i.e., the social environment). Translating this to the child-care setting, this would mean that the influence of the immediate physical child-care environment on child physical activity is moderated by the contextual influence of the social environment (i.e., staff and peer behaviour).

The current study examined the influence of the social and physical childcare environment on physical activity intensity of 2- and 3-year-olds. We hypothesized that physical activity intensity is influenced by both social and the physical environment, as well as by demographic child characteristics. Furthermore, in line with the ecological perspective (Friedman \& Wachs, 1999; Spence \& Lee, 2003; Wachs, 1992), we hypothesized that the physical environment, social environment and demographic characteristics would interact with each other in determining the children's activity intensity.

\section{Method}

The design of the study is based on a study by Bower and colleagues (2008), who cross-sectionally assessed the effects of child-care environment on physical activity among young children in the US through observations. Adapting this design to the Dutch child-care situation required some changes to the original design and instruments. Dutch child-care centres are for instance smaller and have shorter opening hours than US centres.

\section{Design and procedure}

All nine child-care centres of the child-care coordinating organization MIK (Maatwerk In Kinderopvang; i.e., 'customized child-care') in Maastricht, the Netherlands, were approached to participate in the study. MIK is the largest childcare organization in Maastricht, and gave consent to conduct the study. All childcare centres agreed to participate. The child-care centres catered care for an average of $92(\mathrm{SD}=28)$ children each, of which an estimated $12 \%(\mathrm{SD}=14 \%)$ were of nonDutch origin. On average, there were 5 groups $(\mathrm{SD}=1)$, and 20 staff members $(\mathrm{SD}=6)$ per centre. Parents were informed about the study in a letter, and were able to refuse participation, although none of the parents did so. 
Each child-care centre was visited three times in May and June 2008: once for an interview with the manager of the centre and a rating of the activity opportunities, and twice (one morning and one afternoon) for direct observations of both the child-care environment and the children's activity intensity. The observations were performed by two observers, both trained in using the instruments described below. Children were randomly selected for observations.

Activity intensity and other factors described below were observed by a momentary sampling procedure with observations lasting 15 seconds each. The 30 seconds following the observation period were used to record the observation. This procedure was repeated four times over a period of three minutes for each child. Each child was observed for two non-consecutive blocks of four observations. In total, 10 children were observed per centre per day, resulting in a total of 80 observations. This protocol was implemented on two days for each of the nine centres, during one morning and one afternoon, with at least one week between the two observation days. This resulted in 2880 single observations regarding 180 children ( 2 observers $X \quad 9$ centres $X \quad 2$ days/centre $X$ blocks/child X 4 observations/block).

\section{Instruments and coding}

\section{Physical activity intensity and social environment}

During the observations, physical activity intensity and the social environment were assessed by means of an adapted and translated version of the Observational System for Recording Physical Activity in Children - Preschool Version (OSRAC-P; Brown et al., 2006). In line with the OSRAC-P protocol, mean activity intensity during the observation periods (15 seconds) was assessed on a scale from 1 (sedentary) to 5 (highly active). Activity levels $\leq 2$ were regarded as Sedentary; activity levels $\geq 4$ as Moderate and Vigorous Physical Activity (MVPA; Bower et al., 2008).

In addition to activity intensity, two other OSRAC-P scales assessing social environment were used: group size (i.e., alone, with one peer or one adult, with multiple peers or adults) and prompts (i.e., short verbal messages of others to either promote activity (positive prompt) or discourage activity (negative prompt; Brown et al., 2006). In addition, location (i.e., indoor or outdoor) was recorded. These variables concerned features of the direct environment the child was playing in: e.g., group size was assessed as people the child had direct contact with during playing. The OSRAC-P categories for group size and prompts were rated as either being present during the observed period (1) or not (0). Multiple OSRAC-P variables could be present simultaneously. For instance, a child could be prompted by both a peer and a staff member during the same observation period, and both would be recorded. 


\section{Physical environment}

The physical child-care environment was rated using translated and adapted items of the Environment and Policy Assessment and Observation Instrument (EPAO; Ball et al., 2005; Ward et al., 2008). This was done after the interviews with the managers of each individual child-care centre. The EPAO instrument assesses the accessibility of the physical environment, that is, the presence of activity opportunities.

Two subscales of the EPAO, portable equipment and fixed equipment, and an additional subscale, total playing surface, were used to compute scores for activity opportunities in the physical environment. Total playing surface was rated on a scale from 0 (no playing surface) to 10 (very large surface), with separate assessments for indoors and outdoors. Portable equipment was rated by checking the availability of 9 types of equipment (ball, climbing, floor, jump, push, ride, slide, sand-water, and twirl equipment), allocating 2 points for each available type. The total score for portable equipment was calculated as the sum of the points allocated, divided by 9 and then multiplied by 10 (to obtain a scale from 0 to 20). Fixed equipment was rated and calculated in a similar manner for 11 types of equipment (structured play area, merry-go-round, climb, see-saw, slide, tunnel, balance surface, sandbox, swing, space for free play indoor and space for free play outdoor), also resulting in a scale from 0 to 20. The three subscales (i.e., playing surface, portable equipment and fixed equipment) were summed to calculate separate outdoor and indoor scores for activity opportunities, ranging from 0 to 50 .

\section{Background information}

Background information regarding the child-care centres, such as the number of enrolled children, was recorded during the interview conducted with each manager. In addition, child's gender and age were assessed by asking present staff after the observations were finished. Weather conditions and outdoor temperature were recorded per observation day.

\section{Statistical analyses}

All analyses were performed using SPSS 13.0. The scores of both observers on dichotomous variables (e.g., prompt by staff, yes/no) were combined by coding the variable as being present (1) when one or both observers rated that variable as being present, and coding it as absent (0) when both rated it as being absent. For continuous variables (e.g., activity intensity), the mean of the scores of both observers was calculated. Cohen's kappa was used to determine the inter-rater reliability (IRR) of the two observers. This measure indicates the proportional agreement between two observers, corrected for chance agreement (Field, 2005). Mean IRR of the variables assessed during the observations (i.e., activity intensity, 
location, prompts, group size, gender, age) was .7 ( $S D=.2)$, indicating substantial agreement (Landis \& Koch, 1977).

Various background characteristics were explored using descriptive statistics. Differences in mean activity intensity between males and females, and between 2-year-olds and 3-year-olds, were examined using t-tests. All subsequent analyses were performed separately for indoor and outdoor observations. The distribution of the environmental factors, and the mean activity intensity corresponding to these factors were explored.

Stepwise multilevel linear model analyses with 3 levels (i.e., measurement level; child level; centre level) were executed to examine the association between activity intensity and the physical (i.e., activity opportunities, EPAO) and social environment (i.e., peer and staff group size and prompting), while modelling the interdependence between observations within individuals and between individuals within child-care centres. The EPAO score was standardized for all multilevel analyses, in order to ease interpretation. Reference categories for prompts and group size were 'no prompts' and 'no peers (staff) being present', respectively. In the starting model, random intercepts at the child and centre level were included, as well as a first-order autoregressive (AR(1)) correlation structure for repeated measures. Furthermore, random slopes at the centre level were included for gender and age, and random slopes at both the centre level and the child level were included for prompts and group size (both for staff and peers). Insignificant random effects were backward removed from the model, starting with the least significant one. When all remaining random effects had a $p$-value $<.10$, the fixed effects were examined.

We conducted various multilevel analyses, for which we separately estimated the random effects (as described above). First, we examined the bivariate relationships between the physical and social environmental factors and the activity intensity, by entering the environmental factors as independent variables in separate analyses. Next, we conducted multivariate analyses with all independent variables (social and physical environment, child characteristics) simultaneously, in order to correct for possible confounding. Finally, additional multilevel analyses were conducted to test for interaction effects of demographic factors and the social and physical environment in determining physical activity intensity, by adding interaction terms to the analyses. Non-significant interaction terms were deleted one by one in the order of their significance, starting with the least significant interaction. Post-hoc analyses were performed to examine the significant interaction terms, using the methods described by Aiken \& West (Aiken \& West, 1991). Pvalues $<.05$ were considered statistically significant for all fixed effects. 


\section{Results}

Five children were not present during the observations, leading to a total of 175 children that were each observed eight times. This resulted in 2800 single observations (1400 per observer). Data regarding 18 observations periods were missing because these children were absent during one or more of the eight observation periods (e.g., because parents had already picked them up), which left 1382 observation periods for analyses. Eighty-nine (50.9\%) of the observed children were male. The mean age was 2.6 years, with 75 two-year-olds (42.9\%), and 100 three-year-olds $(57.1 \%)$. The mean outdoor temperature during the observations was $20.4^{\circ} \mathrm{C}$ (range: $14^{\circ} \mathrm{C}-26^{\circ} \mathrm{C}$ ). Most of the time the weather was sunny with clear skies (37.7\%); least prevalent weather type was rain $(11.4 \%)$.

\section{Physical activity intensity}

The mean activity intensity level during indoor observations (as assessed by the OSRAC-P, scale 1-5) was 2.36 . Only $5.5 \%$ of indoor physical activity observations were classified as moderate and vigorous physical activity (MVPA; activity level $\geq$ 4), whereas $59.4 \%$ were classified as sedentary behaviour (activity level $\leq 2$ ). Outdoors, the mean activity level was 2.82, with 21.3\% being MVPA, and 31.2\% being sedentary behaviour. There were no significant differences in mean activity intensity level between males and females (activity intensity 2.57 vs. 2.61, respectively; $\mathrm{p}>.05$ ), or between 2-year-olds and 3-year-olds (activity intensity 2.58 vs. 2.59 , respectively; $\mathrm{p}>.05$ ).

\section{Physical and social environment}

The mean indoor EPAO score (representing activity opportunities; scale 0-50) was $23.2(\mathrm{SD}=7.9)$, while the mean outdoor EPAO score was $30.8(\mathrm{SD}=8.7)$. Table 8.1 shows the prevalence of the social environment characteristics. Children most frequently performed an activity together with several other children, and most children were not prompted to engage in a particular behaviour. When a prompt was used, indoors this was more often done by a staff member, while outdoors children were about equally often prompted by staff and peers. 


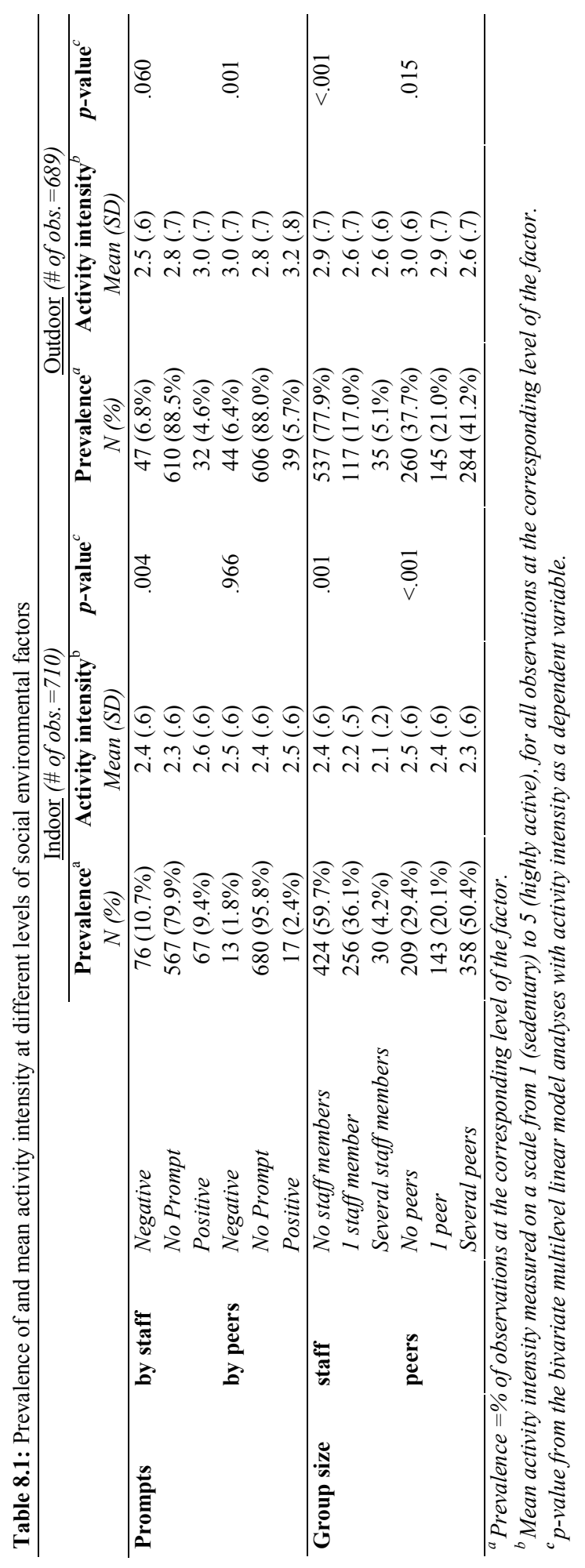


The bivariate multilevel analyses showed that the presence of more activity opportunities (EPAO) was significantly associated with higher activity intensities, both indoors $(\mathrm{B}=.07, \mathrm{p}<.05)$ and outdoors $(\mathrm{B}=.13, \mathrm{p}<.01)$. Table 8.1 shows the mean activity intensities observed in the presence of the various social environment factors. Two social factors were not significantly associated with activity intensity: indoor prompts by peers and outdoor prompts by staff. The other social factors (e.g., group size) showed significant differences $(p<.05)$ in activity intensity between the categories. Both indoors and outdoors, larger group size (both of peers and staff) was associated with lower activity intensity of the child.

\section{Indoor environment and activity intensity}

The multivariate analyses showed that also after correction for co-variates, physical environment activity opportunities were significantly and positively associated with the indoor activity intensity (Table 8.2). Positive prompts by staff were positively related with activity intensity. In addition, group size with regard to staff and peers had a negative influence: the larger the group a child was playing in, the less active the child was.

The association between peer prompting and physical activity intensity was found to be moderated by the child's gender $(\mathrm{p}<.05)$. Post-hoc analyses showed that in girls both negative and positive prompts had a tendency (both $p$ 's $<.10$ ) to be negatively associated with activity intensity $(\mathrm{B}=-.33$ and $\mathrm{B}=-.34$, respectively), but in boys peer prompting was unrelated to activity intensity. Another significant interaction was found between staff group size and child's age $(\mathrm{p}<.05)$. The activity intensity of two-year-olds was more negatively influenced by one $(B=-.45, p<.001)$ or more $(\mathrm{B}=-.38, \mathrm{p}<.05)$ staff members being present, than that of 3 -year-olds $(\mathrm{B}=-$ $.14, \mathrm{p}<.05$ and $\mathrm{B}=-.34, \mathrm{p}<.10$, respectively).

\section{Outdoor environment and activity intensity}

The presence of activity opportunities in the physical environment had a significant positive influence on the outdoor activity intensity, as did positive prompts by staff and peers (Table 8.2). Larger peer group size had a negative influence on the children's physical activity intensity.

Peer group size moderated the influence of activity opportunities on children's activity intensity $(\mathrm{p}<.01)$ : outdoor physical environment had a significant influence only when children engaged in an activity together with multiple other peers $(\mathrm{B}=.22, \mathrm{p}<.001)$. When they played alone or with one other child, this influence was non-significant $(\mathrm{p}>$.05). A significant interaction was also found between prompts by peers and gender $(p<.05)$. Boys were found to respond more positively to peer prompting than girls. Negative peer prompts had a significant 
positive effect in boys $(\mathrm{B}=.28, \mathrm{p}<.05)$, but not in girls $(\mathrm{B}=-.24, \mathrm{p}>.05)$. Positive peer prompts had a stronger effect in boys compared to girls $(\mathrm{B}=.51$ and $\mathrm{B}=.38$, respectively; $\mathrm{p}<.01$ for both). A final significant interaction was found between peer group size and age $(\mathrm{p}<.01)$. Three-year-olds' physical activity intensity was negatively influenced by one $(\mathrm{B}=-.21, \mathrm{p}<.05)$ or more $(\mathrm{B}=-.49, \mathrm{p}<.001)$ peers being present, while for 2-year-olds peer group size was not significantly related to activity intensity $(\mathrm{p}>.05)$.

Table 8.2: Association of environment and demographic child characteristics with physical activity intensity

\begin{tabular}{|c|c|c|c|}
\hline & & $\left.\underline{\text { Indoor }}^{a}{ }_{(\# \text { of obs. }}^{=}=710\right)$ & $\begin{array}{c}\text { Outdoor }^{b} \\
(\# \text { of obs. }=689)\end{array}$ \\
\hline & & \multicolumn{2}{|c|}{ Regression coefficient $(B)$} \\
\hline Gender $($ male $=1$, female $=2)$ & & .10 & -.01 \\
\hline Age (2-3) & & .01 & $.18^{*}$ \\
\hline $\begin{array}{l}\text { Standardized Indoor / Outdoor activity } \\
\text { opportunities (EPAO) score }\end{array}$ & & $.07 *$ & $.11^{*}$ \\
\hline \multirow[t]{2}{*}{$\begin{array}{l}\text { Prompts by staff } \\
(\text { reference category }=\text { no prompt })\end{array}$} & Negative prompt & .12 & -.05 \\
\hline & Positive prompt & $.37 * * *$ & $.39 * *$ \\
\hline \multirow[t]{2}{*}{$\begin{array}{l}\text { Prompts by peers } \\
\text { (reference category = no prompt) }\end{array}$} & Negative prompt & -.05 & .06 \\
\hline & Positive prompt & -.01 & $.42 * * *$ \\
\hline \multirow[t]{2}{*}{$\begin{array}{l}\text { Group size - } \mathbf{s t a f f} \\
(\text { reference category }=\text { no staff members present })\end{array}$} & 1 staff member & $-.24 * * *$ & -.12 \\
\hline & $\begin{array}{l}\text { several staff } \\
\text { members }\end{array}$ & $-.33 * *$ & -.10 \\
\hline \multirow[t]{2}{*}{$\begin{array}{l}\text { Group size - peers } \\
(\text { reference category }=\text { no peers present })\end{array}$} & 1 peer & -.08 & -.13 \\
\hline & several peers & $-.14^{*}$ & $-.32 * * *$ \\
\hline
\end{tabular}

\footnotetext{
$* p<.05, * * p<.01, * * * p<.001$

${ }^{a}$ Final model comprises a random intercept and a random slope for 'Prompts by staff' at the level of the child, and a first-order autoregressive (AR(1)) correlation for repeated measures.

${ }^{b}$ Final model comprises a random intercept at the level of the child-care centre.

Results of multivariate multilevel linear regression analyses. Insignificant ( $p>.10)$ random coefficients were backward deleted. EPAO = Environment and Policy Assessment and Observation instrument, i.e., activity opportunities in the physical environment. Activity intensity was measured on a scale from 1 (sedentary) to 5 (highly active).
} 


\section{Discussion}

The current study examined the influence of the child-care environment on physical activity in 2- and 3-year-olds. A large proportion of the observed activities $(59.4 \%$ of the indoor and $31.2 \%$ of the outdoor observations) were classified as sedentary, while only $5.5 \%$ of the indoor and $21.3 \%$ of the outdoor observations were classified as moderate and vigorous physical activity (MVPA). As hypothesized, the presence of activity opportunities in the physical child-care environment (i.e., the EPAO score) was positively related to the physical activity intensity, both indoors and outdoors. This is in line with the findings of Bower et al. (2008). In addition, the current study showed that social factors were also associated with the activity intensity: staff behaviour and group size were important explanatory factors for the variation in children's physical activity intensity.

In the outdoor environment, the activity opportunities had a significant influence only on children performing an activity together with several peers. Children playing alone or with one other peer may be more concentrated on their playing, and therefore take less notice of their surrounding environment. An alternative explanation might be that the environment is more extensively involved and utilized in group play, compared to when a child plays alone. When staff for instance organizes a game, this is mostly group play, and many children's games utilize the physical environment (e.g., hide and seek, ballgames, etc). The finding that the social environment interacts with the physical environment in determining the child's physical activity intensity, is in line with the ecological 'systems' view of environmental influences on health behaviour (Friedman \& Wachs, 1999; Spence \& Lee, 2003; Wachs, 1992). In addition, several of the social influences on activity found in the current study, were moderated by child characteristics (age and gender). These findings further support models that hypothesize that environmental impact on physical activity is moderated by person-related factors (e.g., Kremers et al., 2006; Spence \& Lee, 2003). The environment seems to have different effects on different individuals, which warrants caution when generalizing results of studies examining environmental influences on behaviour.

One of the identified social influences on physical activity intensity was the presence of staff members. Children were less active when more staff members were present indoors. Closer examination revealed that this only concerned the 2year-olds, and not the 3-year-olds. Previous research also showed this negative association between teachers being present and activity levels (Brown et al., 2009; Cardon et al., 2008), though one study only found this association in girls (Cardon et al., 2008). An explanation for this inverse relationship is that young children, especially girls, prefer to stay close to their teacher, decreasing activity levels when more teachers are present (Cardon et al., 2008). In line with this, we believe that the younger children in the current study might also 'cling' more to their teachers than the older children. Moreover, present preschool staff appear to be relatively 
uninvolved in children's physical activity (Brown et al., 2009), possibly contributing to the negative association between staff being present and children's activity levels. Similar to the findings with regard to the staff group size, the number of peers present also showed a negative relationship with activity in the present study, which is also in line with previous research (Brown et al., 2009).

Positive prompts by staff were found to be positively associated with children's physical activity intensity. In contrast, a previous study by Zask et al. (2001) did not show any effects of staff's encouragement of physical activity. However, staff's participation in activities was included in their measure of encouragement, in addition to verbal encouragement and feedback. It is probable that combining these factors has nullified the effects, since the current study and previous research (Brown et al., 2009; Cardon et al., 2008) have indicated that the number of staff members present is negatively related to physical activity. In line with prompts by staff, positive prompts by peers were positively associated with outdoor activity intensity, while negative prompts showed no effect. However, posthoc analyses based on significant interaction terms showed that girls and boys responded differentially to prompts by peers. In general, in terms of physical activity intensity, it appeared that girls responded less positively to peer prompting than boys. In fact, indoors, any type of peer prompting (both negative and positive) was associated with lower activity intensity in girls, while outdoors both types of peer prompting were associated with higher activity intensity in boys. Because of the cross-sectional nature of the data, we cannot ascertain whether these children's activity levels changed in response to the prompts, or whether their behaviour preceded and perhaps evoked these prompts. More studies are needed to clarify the moderation effect of gender on the influence of peer prompts.

A strong point of the current study is that the environment and physical activity intensity were directly observed, and that it did not rely on other, less valid measures, such as self-report. An additional advantage of the observation format was that we recorded not only activity intensity, but also potential determinants of the behaviour, such as the social context. This provided valuable information on the 'how and what' of the behaviour, in addition to the 'how much'. However, observations are subjective, and might introduce bias, although all observations were performed by 2 observers and inter-observer reliability indicated substantial agreement (Landis \& Koch, 1977). Furthermore, although we based the choice for the length of the observation period ( 15 seconds) on an earlier study using the same instruments (Bower et al., 2008), these periods may have been either too short to record all relevant contextual factors, or too long, leading to uncertainty about the order of events during the observation period. The latter could partially explain the counterintuitive findings of the post-hoc analyses regarding prompts by peers. Another point of consideration is whether the total observed time is representative for a child's activity during a whole day at child-care. However, observations were performed in each child-care centre during both a morning and an afternoon, which 
covers a regular day at child-care in the Netherlands (9:00 AM - 5:00 PM). Finally, children's behaviour could have been influenced by other variables that were not taken into account in this study. For instance, child-care staff's educational level has been previously linked to children's physical activity (Dowda et al., 2004), but was not taken into account in the current study.

The various interactions between environmental and child characteristics found in this study indicate that research on the influence of the environment on physical activity behaviour cannot be restricted to the direct effects of the environment on behaviour, without acknowledging contextual factors. Interactions between and within the social and physical environment should be further examined, in order to confirm the validity of ecological perspectives on determinants of physical activity (Friedman \& Wachs, 1999; Kremers, de Bruijn, Droomers, van Lenthe, \& Brug, 2007; Kremers et al., 2006; Kremers et al., 2008; Spence \& Lee, 2003). Moreover, this perspective should not be restricted to the age group and setting of the current study, as each specific behaviour is the result of the complex interplay of personal and environmental factors.

The current findings stress that the child-care setting could play an important part in the prevention of childhood obesity, and we feel that it deserves more attention than it is currently being granted. A greater understanding of the characteristics of young children and their environment at child-care, would help to identify ways of encouraging more activity in the child-care setting. 
Chapter 9: General discussion 


\section{Introduction}

The aim of this dissertation was to examine the direct and interactive effects of specific features of micro environments on young children's energy balance-related behaviours (EBRBs, i.e., dietary intake and physical activity) and weight status. The micro environments that were examined were those in which young children up to 5 years of age spend most of their time, i.e., the home environment and the childcare environment.

This chapter will provide an overview of the dissertation. First, the main findings of the seven studies that were performed are described. Second, some methodological issues that need to be kept in mind when interpreting the findings are discussed. Then, the implications of the findings are described, both for practice and for theory. Finally, some general conclusions are drawn and directions for future research are presented.

\section{Main findings}

\section{Part 1: The home environment}

The first part of this dissertation discusses the role of the home environment in the development of young children's energy balance-related habits (i.e., dietary intake and physical activity habits), as well as its role in the development of their weight status. Particularly, the role of parents in this respect is examined, using data from the KOALA Birth Cohort Study (Kummeling et al., 2005).

Chapter 2 addresses the influence of breast-feeding duration and feeding pattern on young children's weight development up to the age of 4 years. Parents play a crucial role in shaping an infant's first encounter with food: they decide whether they provide their child with breast-milk or bottle-milk, they are largely responsible for the amount of milk that is provided to the child, and they decide on the timing of the feedings. Research has indicated that breast-feeding may have a long lasting desirable impact on children's weight status (Arenz et al., 2004; Harder et al., 2005; Owen et al., 2005). The mechanisms through which breast-feeding protects against overweight development are, however, still mostly unclear. Hypothesized mechanisms include both biological and behavioural mechanisms (Arenz et al., 2004; Bartok \& Ventura, 2009; Harder et al., 2005). The study described in Chapter 2 confirms the protective effect of breast-feeding against overweight development. In addition, it shows that the feeding pattern (i.e., feeding on demand of the child vs. according to a fixed time schedule) is not a plausible mechanism through which this protective effect might operate, as has previously been suggested (Gillman et al., 2001). Although we did not observe an effect of breast-feeding on overweight in the longer run (at age 5), the finding of an inverse association between breast-feeding and snacking may be a possible mechanism for 
the protective effect of breast-feeding on overweight at a later age. This is in line with previous research (Scholtens et al., 2008).

Chapter 3 examines the clustering or co-occurrence of dietary intake and physical activity in 2-year-old children. Although it has been repeatedly shown that clustering of EBRBs exists in older children and adolescents (e.g., Kremers et al., 2004), research in very young children is lacking. Chapter 3 shows that cooccurence of EBRBs is already present in 2-year-olds: healthy intake behaviours, such as fruit, vegetable and brown bread intake, were found to cluster. In addition, a second cluster was found, in which sedentary behaviour (i.e., computer use and television viewing) is associated with unhealthy intake behaviour (i.e., snack and soft drink intake). Moreover, Chapter 3 shows that these clusters are related to maternal characteristics: children of obese and lower educated mothers more often showed behaviour within the unhealthy 'sedentary-snacking' cluster, and scored less well on the healthy cluster.

Chapter 4 describes the influence of diet-related restrictive parenting practices on 2-year-olds' dietary intake. Many studies have examined the effects of restriction on diet, showing contradicting results: some studies show a desirable effect, with restriction leading to less consumption of that food item (e.g., De Bruijn et al., 2007), while others show a paradoxically increased consumption of the restricted items (e.g., Fisher \& Birch, 1999). Chapter 4 shows a desirable impact of restrictive parenting practices on the children's intake. However, further examination showed that this desirable influence was often stronger or even uniquely present in children with 'favourable' characteristics: a normal eating style, a normal weight and a non-deviant behavioural style (i.e., children scoring low on depressed, anxious and overactive behavioural scales). This interaction between parenting practices and child characteristics might be the explanation for the conflicting finding reported in this research area.

Chapter 5 examines the influence of parenting practices on both dietary intake and physical activity of 5-year-olds. In addition, the influence on weight development between age 5 and age 7 was studied. The study examined the influence of three different parenting practices: restriction, monitoring and stimulation, both with regard to dietary intake and with regard to physical activity. Monitoring a child's dietary intake and stimulation to eat healthy were both associated with desirable effects on children's dietary intake. Restriction of sedentary time was perhaps paradoxically found to be associated with increased sedentary behaviour and decreased physical activity, while stimulation to be active was found to have desirable effects. In line with this finding, BMI development was positively associated with restriction of sedentary time, and negatively associated with stimulation to eat healthy. Similar to the findings in Chapter 4, moderation by child-characteristics was found. Restriction showed undesirable effects in children with a deviant eating style (i.e., relatively hungry or picky eaters), and the desirable effects of monitoring were not present in these children. Restriction and monitoring 
therefore seem inappropriate for children with deviant eating styles. By contrast, stimulation seemed to work especially well for these children. This indicates the need to tune parenting to the individual child, applying 'child-appropriate parenting'.

\section{Part 2: The child-care environment}

The second part of the current dissertation describes the influence of centre-based child-care environments on children's EBRBs and weight status. Data from the KOALA Birth Cohort Study (Kummeling et al., 2005), as well as from observational studies were used for this.

Chapter 6 discusses the influence of child-care use at a young age on weight development up to the age of 2 years. In line with previous studies (Benjamin et al., 2009; Maher et al., 2008), the findings suggest a small but positive influence of child-care use on overweight development, indicating an increased risk of overweight in children who attend child-care compared to those that do not attend child-care. Moreover, it was shown that the intensity of the child-care use did not influence the strength of this relationship. Unfortunately, no information regarding specific environmental features at the child-care centres was known from this study and previous studies. Chapters 7 and 8 therefore examine these environmental features in more detail.

Chapter 7 describes an observational study in the child-care setting, examining children's dietary intake at child-care, and its association with staff behaviour. Children were found to eat more when staff ate together with them, as well as when a family serving style was used (in which the child can self-serve the food). Both findings seem to contradict earlier research, stating that a family serving style is desirable (Fisher et al., 2003; Johnson \& Birch, 1994), and that teacher modelling is unrelated to child dietary intake (Hendy \& Raudenbush, 2000). These seemingly conflicting findings can probably be subscribed to the young age of the children in our study (2- and 3-year-old). Staff discussing healthy foods with the children was found to be associated with a higher fibre intake.

Chapter 8 shows that both the social and the physical child-care environment were significantly related to children's physical activity at child-care. Moreover, in line with an ecological perspective of environmental influences on behaviour (Friedman \& Wachs, 1999; Spence \& Lee, 2003; Wachs, 1992), interactions were found between the social and the physical environment, as well as between the environment and characteristics of the child (i.e., age, gender). For example, peer group size had a negative effect on 3-year-olds' outdoor physical activity level, but had no effect on 2-year-olds. Another example was that the availability of activity opportunities in the outdoor physical environment only had an effect on the activity level of children playing in a group, and not on children playing by themselves or with only one peer. 


\section{Methodological issues}

The different studies described in the current dissertation are subject to various methodological issues, concerning the study populations, assessment choices and the explained variance of the behaviours. The findings of the studies must therefore be interpreted in light of these issues, which are described below.

\section{Study populations}

Although three of the studies presented in this dissertation used a longitudinal design (Chapters 2, 5 and 6), several others used a cross-sectional study design (i.e., Chapters 3, 4, 7 and 8), in which data are collected at only one point in time. A drawback of such a cross-sectional design is that inferences about causality cannot be made. In the case of Chapter 4, for example, we cannot ascertain whether the behaviour of a specific child is influenced by certain parenting practices, or whether the child's behaviour actually evokes these parenting practices. Probably, the influence is bi-directional. Birch and Fisher (2000) have proposed a model in which child weight status predicts diet-related parenting practices, but in which parenting practices also influence the child's weight, through their influence on energy intake (Birch \& Fisher, 2000). A similar bi-directional influence is likely to occur in many instances where a child interacts with his or her environment. The use of longitudinal study designs provides more certainty about the order in which the examined events take place (Pollit \& Beck, 2004).

The majority of the chapters (i.e., Chapters 2 to 6) were based on the KOALA Birth Cohort Study. A specific feature of this cohort is that part of the included parents were recruited through so called 'alternative lifestyle' channels. These alternative recruitment channels included for instance anthroposophist midwives and general practitioners, Steiner schools and organic food shops (Kummeling et al., 2005). The respondents who were recruited through these channels $(17.9 \%)$, could have an alternative lifestyle in terms of for instance dietary habits (e.g., preferring organic foods), child rearing, vaccination schemes or medicine use. However, such an alternative lifestyle was not a criterion for inclusion of the respondents recruited through these channels, nor was an alternative lifestyle a reason to exclude respondents that were recruited via the 'conventional' way (i.e., from an existing cohort for the study of pregnancy-related pelvic girdle pain). Alternative recruitment channel can therefore not be equated with alternative lifestyle, although a high correlation between the two is probable. In addition, the diversity within the group of alternative respondents is large, as the 'alternative' aspect can relate to many different parts of life. For the current dissertation, one can imagine that pursuing an alternative diet or having an alternative viewpoint on child rearing might have consequences for the findings, 
while the influence of pursuing an alternative vaccination scheme is probably negligible. All analyses in the current dissertation that were based on the KOALA cohort were corrected for recruitment channel. Nevertheless, the results of the studies based on the KOALA cohort cannot be simply generalised to other populations.

The use of the KOALA cohort in the current project poses an additional issue of consideration. As do many cohort studies, the KOALA cohort faced dropout of participants, which is of specific concern for the longitudinal studies. At the start, 2834 women were included in the study. At the follow-up moment at the child's age of 5 years, i.e., the fifth follow-up round (follow-ups took place at the child's age of 3 and 7 months, and at 1, 2 and 5 years), 2026 parent-child combinations $(71.7 \%)$ were still participating in the study. This attrition rate is relatively low or comparable to that of similar cohort studies (Environmental Health Risks in European Birth Cohorts (ENRIECO), 2010). Within the KOALA study, much attention is paid to keeping participants involved: they receive regular newsletters for instance, and there is a website for the participants (www.koalastudy.nl). Moreover, after each questionnaire is sent several actions are performed to increase the response rate, for instance by reminder letters, emails and phone calls. These are time consuming measures, but they pay off by keeping response rates high and attrition low. Moreover, although the prevalence of overweight and the mean BMI z-score of the participating children decreased with each follow-up round, secondary analyses showed that there was no selective attrition of overweight children. Children that dropped out before age 5 even had a slightly lower birth weight than the children that did not drop out (see Chapter 2). It seems that the participating children got healthier, in terms of weight status, throughout the follow-up period. This might be attributable to the testing effect of the questionnaires and other measurements within the study, increasing attention of the parents for the topics studied, and possibly influencing their behaviour. This testing effect is a common problem in this research area, especially when self reports are used (Pollit \& Beck, 2004). Alternatively, it could be that the increased awareness of the parents for the topic of energy balance, led to more social desirability in their answers. Social desirability refers to the tendency of respondents to give answers that are congruent with prevailing social values (Pollit \& Beck, 2004). As the current social norm is that (childhood) overweight is undesirable (see e.g., Bauer, Yang, \& Austin, 2004; Davison \& Birch, 2004; Latner \& Stunkard, 2003), this social desirability has possibly resulted in more underreporting of children's BMI in the KOALA Study.

Chapters 7 and 8 were not based on the KOALA Birth Cohort Study, but on observations in various child-care centres. All nine child-care centres participating in these observations were part of the same coordinating organization, 'Maatwerk In Kinderopvang' (MIK). Moreover, all child-care centres were located in Maastricht. As a result, the variation in environmental factors between the child- 
care centres was probably much smaller than the variation between child-care centres throughout the Netherlands or Europe. This limited variation is probably aggravated by the fact that MIK has a shared policy for all child-care centres, which includes some guidelines with regard to dietary intake and physical activity. As a consequence, it is probable that there are other environmental factors that play a role in EBRB at child-care, which could not be detected in the studies described in Chapters 7 and 8.

\section{Assessment issues: Questionnaires}

Chapters 2 to 6 were based on questionnaire data. Some of the most important consequences of this methodology will be described below.

\section{Weight status and overweight}

The questionnaires were all filled out by the parents of the participating children. All data, including child anthropometrics, were therefore parent-reported, and could be biased. Previous research has shown that parental reports of weight and height showed only small differences from measured weight and height. However, parents especially tended to underestimate overweight children's weight (Scholtens et al., 2007). This possibly also resulted in underestimation of children's BMI and overweight in the studies that used parent-reported weight and height in the current dissertation. However, the questionnaires at age 1 and 2 years were completed shortly after the regular visits to infant welfare centres, and parents were asked to report the results of the measurements taken there.

In addition to questioning the validity of parent reported BMI, one should also consider the shortcomings of BMI itself as a measure for assessing childhood overweight. BMI is calculated as a person's weight (in $\mathrm{kg}$ ) divided by the person's squared height (in $\mathrm{m}^{2}$ ). Although BMI is indicated as a useful tool for indicating overweight (Barlow, 2007), one of the main issues is that it does not discriminate between lean mass and fat mass. The use of additional measures, such as waist circumference, is recommended to assess childhood overweight (McCarthy, 2006). Such additional measures were, however, not used in the current dissertation, although they were assessed in a smaller part of the cohort. Moreover, in young children there are no clear cut-off points at which BMI a child is considered overweight or obese. In adults, a person with a BMI above $25 \mathrm{~kg} / \mathrm{m}^{2}$ is considered overweight, and a BMI above $30 \mathrm{~kg} / \mathrm{m}^{2}$ is considered obese. However, children's BMI cut-offs are dependent on their gender, and furthermore change with age. Two different methods are commonly used to create cut-offs for overweight in children. First, Cole and colleagues (2000) published gender-specific cut-off values for ages between 2 and 18 years old, specified to half years (Cole et al., 2000). For developing these cut-offs, anthropometric data regarding weight and height regarding almost 200.000 children were gathered from 6 different countries over the 
world. Centile curves were drawn, extrapolating the cut-off points for adults (i.e., 25 and $30 \mathrm{~kg} / \mathrm{m}^{2}$ ). This resulted in ready to use BMI cut-off points for children and adolescents. But, as Cole and colleagues (2000) indicate themselves, the reference population they used, from only 6 countries, is less than ideal, as it probably reflects Western populations more than it reflects the world's population of children. A second approach for defining cut-offs for childhood overweight does not rely on averaged data from different countries, but bases the cut-off point for childhood overweight on a reference population from the own country (Barlow, 2007), in our case a Dutch reference population. Although there has been some discussion at which percentile of the reference population the cut-offs should lie, general agreement nowadays is that a BMI above the $85^{\text {th }}$ percentile is considered overweight, and a BMI above the $95^{\text {th }}$ percentile is considered obese (Barlow, 2007). A drawback of using this second approach is that the cut-off points change as the reference population changes over time. If the BMI of the reference population increases, the cut-off points for overweight and obesity will also be at a higher BMI. This country-specific reference group approach was applied in most studies of the current dissertation, using data of the Fourth Dutch National Growth Study (Fredriks et al., 2000) as a reference population. As a consequence, findings might have been slightly different if the cut-offs by Cole and colleagues (2000) would have been used.

\section{Energy balance-related behaviours}

The children's EBRBs were also assessed with questionnaires. The questions used to assess dietary intake at the age of 2 years (see Chapters 3 and 4) were not optimal: parents were asked to indicate how often their child consumed a limited number of food items, including various snacks. As portion size was not assessed, we were only able to determine intake frequency of the food items. To be able to provide more detailed information regarding dietary intake, we used a Food Frequency Questionnaire (FFQ) at the age of 5 years, which was validated using doubly labelled water (see Chapter 5; Dutman et al., 2010). The FFQ assessed both intake frequency and portion size of a large variety of food items, intended to estimate the child's energy intake. In addition to dietary intake, the 5 year questionnaire also assessed the children's regular physical activity. Standardized questions from the Dutch Youth Health Care were used for this purpose (Monitor Jeugdgezondheid, 2009).

\section{Parenting practices}

In addition to dietary intake and physical activity, an adjustment was also made between the 2 year and 5 year questionnaire with regard to the assessment of parenting practices. At the 2 year questionnaire, parenting practices were assessed using one question that asked parents whether there were any specific foods that they did not allow their child to consume. The questionnaire stated a number of 
food items (e.g., cookies, sweets, soft drinks) for which this question could be answered (see Chapter 4). As a result, we were only able to draw a conclusion with regard to the parenting practice 'dietary restriction', and only with regard to the specific food items that were asked. At the 5 year questionnaire, we used the validated Child Feeding Questionnaire (Birch et al., 2001) to assess diet-related parenting practices (see Chapter 5). With the use of this questionnaire, we assessed not only 'dietary restriction', but also 'monitoring of diet' and 'stimulation to eat healthy'. In addition, this questionnaire assessed these parenting practices with regard to diet in general, in stead of to specific food items. This resulted in a more complete view of the parenting practices. Also, we converted the diet-related scales to similar physical activity-related scales, to get more insight into parenting practices in relation to physical activity.

Although all other data used in Chapters 2 to 6, such as child-care use, gender and parental educational level, were also self-reported in questionnaires by the parents, we do not have any reasons to believe that this would bias the findings reported in the current dissertation.

\section{Assessment issues: Behavioural observations}

Chapters 7 and 8 were based on observations at child-care centres, posing different assessment issues. Both studies were based on observations during two days. For the study of dietary intake at child-care (Chapter 7), all eating moments were observed only once per child. For the study of physical activity (Chapter 8), children were observed eight times for a short duration. One can wonder whether the observed time in both studies is representative for the total time spent at childcare. However, all meals typically consumed at child-care were included, and physical activity observations were performed at different times spread across a regular day at child-care (9:00-17:00). Moreover, the main focus of both studies was to examine the association of physical activity and dietary intake with the child-care environment, not to provide a representative overview of these behaviours at child-care.

An additional issue to consider with observations is that bias might be introduced by the observers. However, all observations were performed by two observers, with good inter-observer agreement in the dietary intake study (Chapter 7), and substantial agreement in the physical activity study (Chapter 8).

\section{Explained variance}

A final issue to consider when interpreting the findings in the current dissertation is the variance explained by the models presented in the different studies. Although many variables were found to be significant, effect sizes were typically considered at most small, and only a few reached a moderate or large effect size (see e.g., 
Chapter 7, 8; Cohen, 1988). The variance in the EBRBs or weight status explained by the predictors was thus limited. Small to moderate effect sizes are not uncommon in research examining environmental influences on EBRBs (Ferreira et al., 2007; Van der Horst et al., 2006). Moreover, each study described in the current dissertation focuses on a small part of the complex totality of environmental and personal factors that determine a child's EBRBs and weight status. In addition, the determinants that were examined were mostly environmental or background variables, which are quite distal from the actual behaviour in the causal chain explaining the behaviour. Environmental factors may for instance exert their influence on behaviour via a mediated pathway of for example cognitive behavioural determinants (Kremers et al., 2006). The fact that the explained variances are relatively low is hence not surprising. Measurement issues concerning both predictors and outcomes in the various studies may also play a role in this respect. With regard to diet-related parenting practices for instance, there is no consensus about the proper way to measure these concepts: there are dozens of questionnaires assessing these concepts, such as the Child Feeding Questionnaire (Birch et al., 2001), the Infant and Preschooler Feeding Questionnaires (Baughcum et al., 2001), and the Comprehensive Feeding Practices Questionnaire (MusherEizenman \& Holub, 2007). With regard to physical activity-related parenting practices there are not even any validated or widely accepted questionnaires available. Similarly, the outcome variables are also difficult to assess. As discussed above, BMI is for instance not an optimal method for childhood overweight assessment (McCarthy, 2006), but more precise methods such as dual-energy x-ray absorptiometry are often very costly and not feasible for large scale implementation. Measurement errors and misclassification due to measurement issues such as the ones described above, lead to underestimation of effect sizes. It is clear that this research area is still developing, and consensus about feasible and valid measurement methods has yet to be established. This will ultimately result in more reliable data, and possibly also larger effect sizes and explained variances.

\section{Implications for practice}

The findings of the current dissertation hold various implications for practice, which will be described below. These practical implications concern an energy balance approach, settings for environmental interventions in children, target groups for such interventions, and the contextual influence of specific situations and specific children.

\section{An energy balance approach}

Traditionally, research into determinants of physical activity or dietary intake has been focused on only one behaviour (Kremers, 2010). However, not single 
behaviours but the combination of multiple risk behaviours determines whether an individual is at a high risk to develop overweight or obesity (Rennie et al., 2005). Chapter 3 confirms this by showing that EBRBs cluster: children who displayed much sedentary behaviour were also more likely to consume many snacks and soft drinks. With the combination of these risk factors, having both a high risk of excess energy intake at the one side of the energy balance, and low energy expenditure at the other side, children displaying these clustered behaviours are especially likely to develop overweight. On the other side, healthy behaviours have also been found to cluster, increasing chances of maintaining a healthy weight.

The finding that EBRBs cluster in youth is not new, as many studies have shown this before (e.g., Kremers et al., 2004). What the current study adds to this evidence base, though, is that it shows such cross-behavioural clustering in children as young as 2 years old (Chapter 3). The findings imply that overweight prevention efforts will need to apply an integrated prevention approach, in which physical activity and dietary intake are addressed simultaneously. Moreover, such preventive efforts will need to target children at a very young age, before clustered obesogenic behaviours have rooted in the child's habits. An example of a preventive intervention taking into account clustering of EBRBs, is that parents could be advised not to offer unhealthy snacks to the child when watching television, in order to prevent that television watching becomes a cue for unhealthy snacking.

In addition, not only the behaviour itself, but also energy balance-related parenting practices have been found to cluster (Chapter 4). As clusters of parenting practices could be indicators of a wider obesogenic parental or family context, the current insights into the clustering of parenting practices can help inform the development of interventions aimed at improving parenting, within an energy balance approach.

\section{Settings for environmental interventions}

The studies described in the current dissertation have indicated two micro environmental settings in which interventions preventing weight gain in young children could take place: the home environment and the child-care setting. The studies have pointed out various specific environmental features of these setting that could possibly be targeted in such interventions.

\section{The home environment}

Central in shaping the child's home environment are the child's parents. One of the most important methods parents have for influencing a child's EBRB is the application of certain parenting practices or rules (Golan \& Crow, 2004). Chapters 4 and 5 have confirmed the importance of parenting practices, and shown that in general, restriction of unhealthy food intake has desirable effects in young children. By contrast, with regard to sedentary behaviour restriction was found to have 
disadvantageous effects on children's behaviour, and is therefore not recommended. A positive parenting approach seems to be particularly beneficial though: parental stimulation to be active and to eat healthy are both associated with desirable effects on children's behaviour. A focus on a positive, stimulating approach is therefore recommended for interventions focusing on parenting practices.

In addition to the rules parents apply, they also have an influence on children's diet on a much more basic level: they determine which foods they provide to their children (Golan \& Crow, 2004). During infancy, children are even totally dependent on what their parents provide them with, i.e., breast-milk, bottlemilk, or (at a later stage) other foods. Chapter 2 has shown the important influence parents thus have. Children that were provided with breast-feeding were less prone to develop overweight, and showed a healthier intake pattern at a later age. Breastfeeding therefore seems to be an important first step in responsive energy-balance parenting. This is confirmed by the fact that previous research has shown that breast-feeding is associated with a less controlling parental feeding style at a later age (Farrow \& Blissett, 2006; Fisher et al., 2000; Taveras et al., 2004), and less negative mealtime interactions and mealtime conflicts (Farrow \& Blissett, 2006).

\section{The child-care environment}

Young children today spend increasing amounts of time in child-care. In Europe, a quarter of the infants and over three quarters of the children up to school age attend some form of child-care or education facilities (Blackburn, 2006; Eurostat, 2008). Child-care staff therefore fulfil to an increasing degree a role in child rearing (De Schipper, Tavecchio, \& Van IJzendoorn, 2007; Van IJzendoorn, Tavecchio, Stams, Verhoeven, \& Reiling, 1998b), implying that the child-care setting could also play an important role in the prevention of overweight and obesity development in young children (Story et al., 2006).

Chapter 6 confirmed the importance of child-care in the development of weight status in young children. In line with previous studies (Benjamin et al., 2009; Maher et al., 2008), we showed that child-care use was associated with increased BMI and overweight risk. However, there is also a limited number of studies showing the opposite, i.e., a protective effect of child-care use (Frisvold \& Lumeng, 2007; Lumeng et al., 2005). The reason for these conflicting findings might lie in differences between the child-care centres in the different studies with regard to the degree to which the environment in the centres could be considered 'obesogenic'. This signifies that child-care use should not be discouraged, but rather, it implies a window of opportunity for preventive interventions in this setting. Chapters 7 and 8 therefore looked into specific environmental features at child-care that are related to children's dietary intake and physical activity. The importance of staff behaviour (i.e., modelling, verbal prompting, stimulation and even mere presence) was shown in both studies, indicating that much can be gained by properly educating child-care staff about their role in shaping children's EBRBs. 
In addition, the importance of the physical child-care environment was shown, indicating that physical activity levels at child-care could improve by providing more activity opportunities (i.e., more and suitable equipment and space for playing).

\section{Target groups for interventions}

In the prevention of health problems, including childhood overweight, three different levels of prevention can be employed: universal prevention (addressing the entire population), selective prevention (focusing on high risk groups), and indicated prevention (focusing on individuals exhibiting early signs of a health problem; Gordon, 1987). In the case of childhood overweight and obesity, universal prevention would aim at improving (determinants of) energy balance-related behaviours and ultimately stabilizing or reducing the mean BMI in the general population of children. Selective prevention would target children with an increased risk of becoming overweight or obese (e.g., children of parents with overweight); and indicated prevention would target children that have a relatively high BMI, or are perhaps already overweight (Muller, Mast, Asbeck, Langnase, \& Grund, 2001). Although all three types of overweight and obesity prevention need further research to establish their (long term) effects, it is plausible that simultaneous efforts regarding each of these types would lead to the best results (Muller et al., 2001).

If one chooses for selective prevention in targeting childhood obesity, several groups of parents and children can be identified as high risk groups, based on the current dissertation. These groups should receive specific attention in childhood overweight prevention efforts.

Several parental characteristics were shown to be related to an increased risk of weight gain. First of all, maternal weight status was shown to be important. Children of obese mothers showed more unhealthy clustered behaviour (i.e., sedentary behaviour, snack and soft drink intake) than children of non-obese mothers (see Chapter 3). Moreover, maternal BMI was negatively correlated with stimulation of healthy food intake at age 5 (Chapter 5). As stimulation was shown to have desirable effects on children, the lack of this stimulation poses an additional risk on children of overweight mothers. Another risk factor is the mother's educational level. Children of lower educated mothers showed high scores on the unhealthy 'sedentary-snacking' cluster, and low scores on the healthy 'fibre' cluster (Chapter 3). In addition, lower educated mothers showed less restriction of dietary intake at age 2 (Chapter 4), which is in line with previous research (Brown et al., 2008; Hupkens et al., 1998). Furthermore, lower educated mothers also used less stimulation with regard to both healthy intake and physical activity at age 5 (Chapter 5), which are all parenting practices that were shown to have desirable effects on children's behaviour. A final parental characteristic that is associated with increased child overweight risk, is long maternal working hours. The number 
of hours that mothers worked was negatively associated with the use of parental practices with desirable effects: monitoring of dietary intake and stimulation of physical activity (Chapter 5). Mothers who are obese, low educated or have long working hours, could thus be considered as relevant target groups for childhood overweight prevention interventions.

As Chapter 7 showed that child-care use was associated with higher BMI, one may conclude that children attending child-care also make up a high risk group. However, as argued above, this seems to be dependent on the obesogenicity of the child-care centre the child attends. Specific features of child-care centres that would pose a high risk on the children attending them, would for instance be child-care centres that do not provide enough activity opportunities, with staff that does not show desirable behaviour themselves and do not encourage the children to eat healthy and be active (see Chapters 8 and 9). Child-care staff could therefore be an important target group for preventive interventions. Furthermore, as child-care use has been found to be positively predicted by parental working hours (Chapter 7), this is an additional reason for considering mothers (and fathers) with long working hours as a relevant target group for interventions.

In addition to identifying high risk groups, another important issue is to identify settings in which these high risk groups can be detected, and in which subsequent preventive interventions could be implemented. In the Dutch health care system all parents are invited for regular periodic screenings of the child at infant welfare centres. As the attendance rate at these screenings is very high (i.e., 95\%; Nationaal Kompas Volksgezondheid, 2008), this seems to be an appropriate setting for identifying high risk groups at this young age, in order to be able to apply selective prevention. In addition, this is also a setting in which universal and indicated overweight prevention methods could be applied. However, feasibility and effectiveness studies would then first need to show the potential impact of implementing such overweight-related interventions in Youth Health Care.

\section{Specific situations and specific children}

Various studies in this dissertation showed that the effects of the environment on children's behaviour are moderated by several factors. These moderating factors included characteristics of the child itself, as well as characteristics of the social environment. This means that future environmental interventions targeting EBRBs in young children should take into account, and perhaps be tailored to, the specific characteristics of the child and the specific situation in which the behaviour is performed.

Chapters 4 and 5 both showed that the effects of parenting practices on children's EBRBs, was dependent on specific characteristics of the child. The desirable effects of diet-related restrictive parenting practices applied by parents of 2-year-olds (see Chapter 4) were found to be less strong or even absent in children 
with a deviant behaviour style (i.e., showing depressed or anxious behaviour), in children with a deviant eating style (i.e., children not liking many foods, eating with reluctance or being a slow eater), and in children with a higher BMI. Restriction even had an undesirable effect on 2-year-olds with an overactive behavioural style, and on 5-year-olds with a deviant eating style (i.e., who were indicated as relatively hungry compared to peers, see Chapter 5). These findings indicate that the use of restriction is useless or even potentially disadvantageous for children who are in some way deviant from a 'normal' child, and it would perhaps be wise to advise parents of such children against the use of such restrictive practices. Instead, parents of these 'deviant' children should better use a positive approach in their energy balance-related parenting, as shown in Chapter 5. Stimulating healthy eating was found to be specifically beneficial for picky eaters and children with a high BMI.

In line with the findings of Chapters 4 and 5, Chapter 8 also reports moderation by child characteristics when it comes to the influence of the social child-care environment on children's physical activity levels. Prompts were found to have different effects on boys and girls, and the effect of the group size the child was playing in was dependent on the child's age. No straightforward recommendations for child-care staff about what to do and what not to do can be deduced from this, as child-care staff often do not interact with a single child but with the whole group of children, including both boys and girls, and both younger and older children. It is important, however, that child-care staff become aware of these age and gender differences. For instance, if staff members keep in mind that younger children tend to 'cling' more to staff members than older children, they can respond to this properly: they can pay specific attention to encouraging these young 'clinging' children to get active again.

\section{Implications for theory and research}

Besides implications for the practice of overweight prevention, the current findings also hold implications for theory and research in the field of environmental influences on EBRBs.

\section{Ecological framework}

The current dissertation was based on an ecological view of environmental influences on behaviour in general (Friedman \& Wachs, 1999; Wachs, 1992) and physical activity in particular (Spence \& Lee, 2003). Already in 1935, Lewin argued that individual functioning cannot be understood without considering the environment in which it occurs (Lewin, 1935). The ecological view assumes that within the so-called micro system dimension, physical environmental factors (i.e., features of the immediate behavioural setting) interact with the actors that operate within this setting (i.e., the social environment). In addition, person-related factors, 
such as demographic factors, are thought to moderate the impact of environmental influences as well (Kremers et al., 2006).

It has been argued that this ecological systems view needs further testing in different settings in order to confirm its validity (Spence \& Lee, 2003). Studies applying such an ecological perspective, examining interactive effects of predictors of EBRBs, are relatively scarce, however (Kremers, de Bruijn et al., 2007), especially in children. The ecological view on environmental influences was adopted in several of the studies described in this dissertation. Chapters 4 and 5, for instance, applied this framework in the examination of the effects of energy balance-related parenting practices. Both studies showed that those effects were dependent on characteristics of the child. This is in line with the Environmental Research framework for weight Gain prevention (EnRG-framework), an ecological dual-process framework (Kremers et al., 2006). The EnRG-framework hypothesizes that the influence of the environment is moderated by person-related factors such as demographic factors and personality. Chapter 4 provided evidence for the moderating role of person-related factors: children with deviant behavioural styles (i.e., children showing depressive, aggressive or anxious behaviour) were less affected by parenting practices than children not showing such a deviant personality. Chapter 8 confirmed the moderating role of demographics, by showing that age and gender moderated environmental effects on the child's physical activity. Another factor that has been shown to have such a moderating role was the weight status of the child (Chapters 4 and 5). The current dissertation further showed the existence of additional moderators in the relationship between environment and EBRBs: diet-related behavioural styles (i.e., picky eating, eating fast) were shown to moderate the influence of parenting practices (see Chapters 4 and 5).

The current dissertation also confirmed the broader ecological perspective on environmental influences. Chapter 8 showed that the social and physical childcare environment interacted with each other in determining children's physical activity levels. However, interaction between the social and physical environment was only examined in the child-care setting. The question arises whether such interactions also take place in the home environment. Studies examining this are however lacking.

\section{Conclusions and future directions}

The current dissertation examined micro-environmental influences on young children's EBRBs, studying both the home environment and the child-care environment, showing that both settings play a crucial role in the development of children's weight status and weight-related habits. Within the social environment, parents, child-care staff and peers are the primary actors for a young child. Their practices and prompts, but even their mere presence, seem to influence children 
dietary intake and physical activity. In the physical environment the availability of opportunities to engage in EBRBs, i.e., to be active or to consume certain foods, are important.

The findings showed that several environmental and personal factors interact with each other in determining EBRBs, both at child-care and at home. This indicates that research into environmental influences on behaviour should not be limited to merely examining direct associations between predictors and outcomes, but should incorporate theory-based examination of possible moderation effects. A theoretical perspective to guide such examinations could be the ecological perspective (Friedman \& Wachs, 1999; Spence \& Lee, 2003; Wachs, 1992), which was the basis for the current dissertation. Following this perspective, moderation effects to be examined are both interactions between multiple types and levels of environmental influences, as well as interactions between the environment and the person performing the behaviour in that environment. On the methodological level, future studies should focus on experimental research designs, in order to confirm and extend the current findings that are mostly based on observational studies (including the studies in the current dissertation).

In the introduction, we presented a research framework for the current study (see Introduction, Figure 1.1). Based on our findings described above, we can say that the larger part of this framework was confirmed. The figure presented below describes the relationships that were confirmed by the current dissertation. The grey parts of Figure 9.1 represent the relationships that were not studied in the current dissertation. One of the topics that was not examined in the current dissertation, is the relationship between EBRBs and weight status. This relationship has, however, been examined many times before, and is well established (see e.g., Rennie et al., 2005; WHO/FAO Expert Consultation, 2003). A second topic that was not examined is the influence of the physical home environment on EBRBs and weight, and its interaction with other variables such as the social home environment and child characteristics. As the first part has been examined before (see e.g., Ezendam, Evans, Stigler, Brug, \& Oenema, 2010; Rosenberg et al., 2010; Timperio et al., 2008), future research should focus on clarifying the second part, i.e., the interactions between types of environments and child characteristics, in line with the ecological view. We hypothesize these interactions to be similar to those that we found at the child-care centre, i.e., that the effect of the physical environment on dietary intake and physical activity is dependent on social factors such as the other people present, and personal factors such as age and gender. 


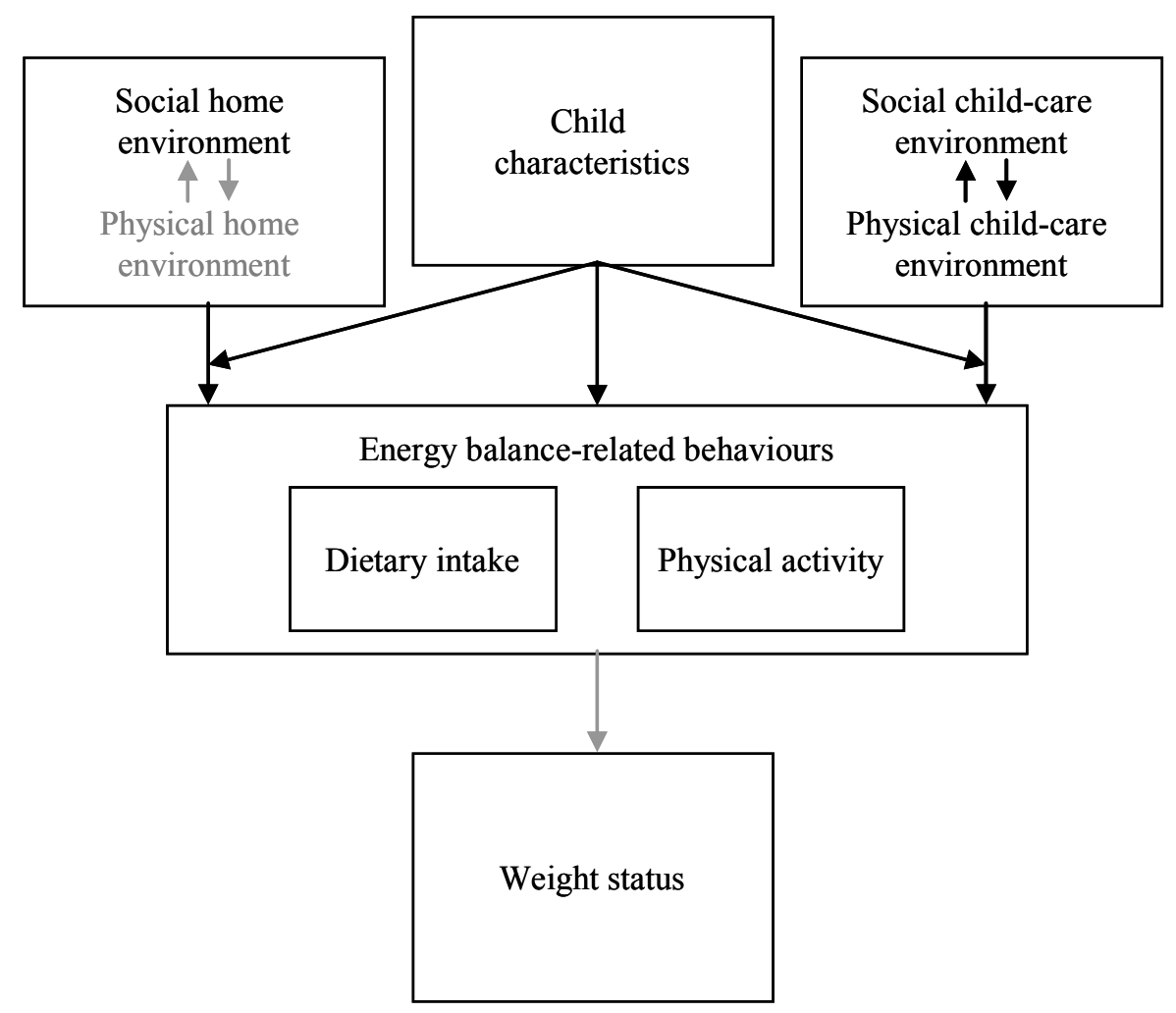

Figure 9.1: Confirmed relations of the research framework of the current dissertation

An additional direction for future research is to look further into the effects of different types of parenting practices, as well as the interactions between these types. The current dissertation focused solely on explicit or overt parenting practices. Overt control is defined as forms of control that can be detected by the child (Brown et al., 2008). However, there are also other types of parenting practices that are of importance in forming EBRBs. Brown and colleagues (2008) introduced the concept of covert control, a type of control that cannot be detected by the child (Brown et al., 2008). Extending this view, we believe one could also distinguish between explicit and implicit parenting practices. Implicit parenting is not only covert for the child, but might also be covert for the parent. In other words, the parent might not even know that this is a factor that influences the child, although it is an important component of energy balance-related parenting as a whole. Examples of such implicit parenting behaviours are parental modelling, concerns and attitudes that they hold with regard to EBRBs and weight. Darling and Steinberg (1993) have argued that general parenting styles provide the context in which specific parenting practices operate (Darling \& Steinberg, 1993). This has been confirmed by studies showing that the influence of parenting practices depends on the parents' more general parenting style (Musher-Eizenman \& Holub, 
2006; Van der Horst et al., 2007). We believe that these interactions might also take place at a more proximal sublevel. Musher-Eizenman and Holub (2006) showed that the effect of restrictive parenting practices is dependent on whether the motivation for these restrictions is health-related or weight-related, as this motivation determines how the child experiences the restriction. They further hypothesized that other contextual variables, such as parental modelling, could also moderate the influence of restriction (Musher-Eizenman \& Holub, 2006). Extending this view, the effects of explicit parenting practices (e.g., restriction, monitoring, encouragement) may be moderated by the effects of the more implicit parenting behaviours as outlined above (e.g., attitudes, concerns, modelling). Our hypothesis with regard to different parenting types and their interactive effects should be further examined in future studies.

An important step for future research is to combine the findings of research in the two settings described in this dissertation. In general, research regarding parental influences on EBRBs and weight is much more extensive and comprehensive than the current evidence base regarding influences in the child-care setting (Story et al., 2006). Many of the findings in the home setting could probably be translated to the child-care setting. Research regarding the role of child-care in overweight development should therefore perhaps be focussed on the testing of hypotheses based on research in the area of parental influences. Many studies have for instance examined the influence of parenting styles on EBRBs and weight (see e.g., Berge, Wall, Loth, \& Neumark-Sztainer, ; Kremers et al., 2003; Rhee, Lumeng, Appugliese, Kaciroti, \& Bradley, 2006; Van der Horst et al., 2007). Research regarding 'supervision styles', as the equivalent of the parenting style in the child-care setting, is however lacking. In a similar way, many other topics that have been thoroughly examined in the home setting could be the basis for future child-care research.

A final issue of consideration for future studies is to examine the interaction and the reciprocity between the home setting and the child-care setting. The current paper examined these settings as separate micro environments that each influence child behaviour individually. In practice however, those micro environments will also influence each other, and it is their combined influence that will determine a child's behaviour and eventually his or her weight status (Bradley, 2010). Bronfenbrenner has called such interaction between two micro systems the meso system (Bronfenbrenner, 1979). Not much is known yet regarding the interaction between home and the child-care settings, although research indicates that parents and child-care staff often have different attitudes, practices and styles when it comes to child-rearing (Tavecchio, IJzendoorn, \& Stams, 1996). Moreover, communication between both parties is often found to be limited (Bradley, 2010). This lack of communication leads us to consider reciprocity between parents and child-care staff that goes beyond them being in direct contact. What will happen when a child is for instance not allowed to watch television before $5 \mathrm{pm}$ at home, 
but is allowed to do so at the child-care centre? And what happens if parents consistently try to model healthy EBRBs for their child, but child-care staff is sedentary and eats unhealthy snacks in presence of the same child? Will this undermine the parental efforts, and what effect does this have on the child's actual behaviour? Does the influence of each of these settings reach beyond the behaviour performed in that setting itself? Or are parental influences on children robust to such interferences from outside the parent-child relationship? Although previous research showed that conformity between parents and child-care staff does not seem to influence the quality of the care provided (Van IJzendoorn, Tavecchio, Stams, Verhoeven, \& Reiling, 1998a), it has also been shown that disparities between parents and staff negatively affect children's overall wellbeing (Tavecchio et al., 1996; Van IJzendoorn et al., 1998b). The consequences of a lack of conformity for EBRBs and weight status are not clear. The influence of meso systems on the child's EBRBs, i.e., in this case the interaction between the child-care setting and the home setting, will therefore need further examination in future research. 


\section{References}

Aarts, C., \& Greiner, T. (2006). Regarding the review article by Erlanson-Albertsson and Zetterstrom, Acta Paediatr 2005;94:1523-31. Acta Paediatr, 95(5), 623-624.

Aiken, L.S., \& West, S.G. (1991). Multiple Regression: Testing and interpreting interactions. Newbury Park, CA: Sage.

American Dietetic Association. (1994). Position of the American Dietetic Association: nutrition standards for child care programs. J Am Diet Assoc, 94(3), 323.

American Dietetic Association and Dieticians of Canada. (2003). Position of the American Dietetic Association and Dieticians of Canada: Vegetarian diets. J Am Diet Assoc, 103(6), 748-765.

Anderson, C.B., Hughes, S.O., \& Fuemmeler, B.F. (2009). Parent-child attitude congruence on type and intensity of physical activity: testing multiple mediators of sedentary behavior in older children. Health Psychol, 28(4), 428-438.

Aranceta, J., Perez-Rodrigo, C., Ribas, L., \& Serra-Majem, L. (2003). Sociodemographic and lifestyle determinants of food patterns in Spanish children and adolescents: the enKid study. Eur $J$ Clin Nutr, 57 Suppl 1, S40-44.

Arenz, S., Ruckerl, R., Koletzko, B., \& von Kries, R. (2004). Breast-feeding and childhood obesity - a systematic review. Int J Obes Relat Metab Disord, 28(10), 1247-1256.

Arredondo, E.M., Elder, J.P., Ayala, G.X., Campbell, N., Baquero, B., \& Duerksen, S. (2006). Is parenting style related to children's healthy eating and physical activity in Latino families? Health Educ Res, 21(6), 862-871.

Balder, E., Ter Doest, D., \& Hulshof, K. (2006). Invloed van toegevoegde suikers op voorziening microvoedinsstoffen (Influence of added sugars on micronutrient supply). Voeding $N U, 4$, 21-24.

Ball, S.C., Benjamin, S.E., Hales, D.P., Marks, J., McWilliams, C.P., \& Ward, D.S. (2005). The Environment and Policy Assessment and Observation (EPAO) child care nutrition and physical activity instrument Center for Health Promotion and Disease Prevention, University of North Carolina at Chapel Hill.

Ball, S.C., Benjamin, S.E., \& Ward, D.S. (2008). Dietary intakes in North Carolina child-care centers: are children meeting current recommendations? J Am Diet Assoc, 108(4), 718-721.

Barlow, S.E. (2007). Expert committee recommendations regarding the prevention, assessment, and treatment of child and adolescent overweight and obesity: summary report. Pediatrics, 120 Suppl 4, S164-192.

Baron, R.M., \& Kenny, D.A. (1986). The moderator-mediator variable distinction in social psychological research: conceptual, strategic, and statistical considerations. J Pers Soc Psychol, 51(6), 1173-1182.

Bartok, C.J., \& Ventura, A.K. (2009). Mechanisms underlying the association between breastfeeding and obesity. Int J Pediatr Obes, 4(4), 196-204.

Bastiaanssen, J.M., de Bie, R.A., Bastiaenen, C.H., Heuts, A., Kroese, M.E., Essed, G.G., \& van den Brandt, P.A. (2005). Etiology and prognosis of pregnancy-related pelvic girdle pain; design of a longitudinal study. BMC Public Health, 5, 1.

Bauer, K.W., Yang, Y.W., \& Austin, S.B. (2004). "How can we stay healthy when you're throwing all of this in front of us?" Findings from focus groups and interviews in middle schools on environmental influences on nutrition and physical activity. Health Educ Behav, 31(1), 3446.

Baughcum, A.E., Powers, S.W., Johnson, S.B., Chamberlin, L.A., Deeks, C.M., Jain, A., \& Whitaker, R.C. (2001). Maternal feeding practices and beliefs and their relationships to overweight in early childhood. J Dev Behav Pediatr, 22(6), 391-408.

Ben-Sefer, E., Ben-Natan, M., \& Ehrenfeld, M. (2009). Childhood obesity: current literature, policy and implications for practice. Int Nurs Rev, 56(2), 166-173. 
Benjamin, S.E., Rifas-Shiman, S.L., Taveras, E.M., Haines, J., Finkelstein, J., Kleinman, K., \& Gillman, M.W. (2009). Early child care and adiposity at ages 1 and 3 years. Pediatrics, 124(2), 555-562.

Berge, J.M., Wall, M., Loth, K., \& Neumark-Sztainer, D. (2010). Parenting style as a predictor of adolescent weight and weight-related behaviors. $J$ Adolescent Health, 46(4), 331-338.

Birch, L., Savage, J.S., \& Ventura, A. (2007). Influences on the Development of Children's Eating Behaviours: From Infancy to Adolescence. Can J Diet Pract Res, 68(1), s1-s56.

Birch, L.L., \& Fisher, J.O. (2000). Mothers' child-feeding practices influence daughters' eating and weight. Am J Clin Nutr, 71(5), 1054-1061.

Birch, L.L., Fisher, J.O., Grimm-Thomas, K., Markey, C.N., Sawyer, R., \& Johnson, S.L. (2001). Confirmatory factor analysis of the Child Feeding Questionnaire: a measure of parental attitudes, beliefs and practices about child feeding and obesity proneness. Appetite, 36(3), 201-210

Blackburn, P. (2006). Childcare services in the EU - what future? European Foundation for the Improvement of Living and Working Conditions.

Boldemann, C., Blennow, M., Dal, H., Martensson, F., Raustorp, A., Yuen, K., \& Wester, U. (2006). Impact of preschool environment upon children's physical activity and sun exposure. Prev Med, 42(4), 301-308.

Bollella, M.C., Spark, A., Boccia, L.A., Nicklas, T.A., Pittman, B.P., \& Williams, C.L. (1999). Nutrient intake of Head Start children: home vs. school. J Am Coll Nutr, 18(2), 108-114.

Bower, J.K., Hales, D.P., Tate, D.F., Rubin, D.A., Benjamin, S.E., \& Ward, D.S. (2008). The Childcare Environment and Children's Physical Activity. Am J Prev Med, 34(1), 23-29.

Boynton-Jarrett, R., Thomas, T.N., Peterson, K.E., Wiecha, J., Sobol, A.M., \& Gortmaker, S.L. (2003). Impact of television viewing patterns on fruit and vegetable consumption among adolescents. Pediatrics, $112(6 \mathrm{Pt}$ 1), 1321-1326.

Bradley, R.H. (2010). From Home to Day Care: Chaos in the Family/Child-Care Mesosystem. In G. W. Evans \& T. D. Wachs (Eds.), Chaos and Its Influence on Children's Development. Washington: American Psychological Association.

Bradley, R.H., \& Vandell, D.L. (2007). Child care and the well-being of children. Arch Pediat Adol Med, 161(7), 669-676.

Brann, L.S., \& Skinner, J.D. (2005). More controlling child-feeding practices are found among parents of boys with an average body mass index compared with parents of boys with a high body mass index. J Am Diet Assoc, 105(9), 1411-1416.

Briley, M.E., Jastrow, S., Vickers, J., \& Roberts-Gray, C. (1999). Dietary intake at child-care centers and away: are parents and care providers working as partners or at cross-purposes? J Am Diet Assoc, 99(8), 950-954.

Bronfenbrenner, U. (1979). The Ecology of Human Development: Experiments by Nature and Design. Cambridge, MA: Harvard University Press.

Brown, K.A., Ogden, J., Vogele, C., \& Gibson, E.L. (2008). The role of parental control practices in explaining children's diet and BMI. Appetite, 50(2-3), 252-259.

Brown, R., \& Ogden, J. (2004). Children's eating attitudes and behaviour: a study of the modelling and control theories of parental influence. Health Educ Res, 19(3), 261-271.

Brown, W.H., Pfeiffer, K.A., McIver, K.L., Dowda, M., Addy, C.L., \& Pate, R.R. (2009). Social and environmental factors associated with preschoolers' nonsedentary physical activity. Child Dev, 80(1), 45-58.

Brown, W.H., Pfeiffer, K.A., McLver, K.L., Dowda, M., Almeida, M.J., \& Pate, R.R. (2006). Assessing preschool children's physical activity: the Observational System for Recording Physical Activity in children-preschool version. Res Q Exercise Sport, 77(2), 167-176.

Brug, J., Oenema, A., \& Ferreira, I. (2005). Theory, evidence and Intervention Mapping to improve behavior nutrition and physical activity interventions. Int J Behav Nutr Phys Act, 2(1), 2.

Burdette, H.L., Whitaker, R.C., Hall, W.C., \& Daniels, S.R. (2006). Breastfeeding, introduction of complementary foods, and adiposity at $5 \mathrm{y}$ of age. Am J Clin Nutr, 83(3), 550-558. 
Butte, N., Cobb, K., Dwyer, J., Graney, L., Heird, W., \& Rickard, K. (2004). The Start Healthy Feeding Guidelines for Infants and Toddlers. J Am Diet Assoc, 104(3), 442-454.

Cardon, G., Labarque, V., Smits, D., \& De Bourdeaudhuij, I. (2009). Promoting physical activity at the pre-school playground: The effects of providing markings and play equipment. Prev Med, 48(4), 335-340.

Cardon, G., Van Cauwenberghe, E., Labarque, V., Haerens, L., \& De Bourdeaudhuij, I. (2008). The contribution of preschool playground factors in explaining children's physical activity during recess. Int J Behav Nutr Phys Act, 5, 11.

Carper, J.L., Orlet Fisher, J., \& Birch, L.L. (2000). Young girls' emerging dietary restraint and disinhibition are related to parental control in child feeding. Appetite, 35(2), 121-129.

Centraal Bureau voor de Statistiek (2005). Beroepsbevolking; geslacht en leeftijd. Voorburg/Heerlen: CBS.

Clark, H.R., Goyder, E., Bissell, P., Blank, L., \& Peters, J. (2007). How do parents' child-feeding behaviours influence child weight? Implications for childhood obesity policy. J Public Health, 29(2), 132-141.

Cohen, J. (1988). Statistical power analysis for the behavioral sciences. New York: Hillsdale, Erlbaum.

Cole, T.J., Bellizzi, M.C., Flegal, K.M., \& Dietz, W.H. (2000). Establishing a standard definition for child overweight and obesity worldwide: international survey. Bmj, 320(7244), 1240-1243.

Cooke, L.J., Wardle, J., Gibson, E.L., Sapochnik, M., Sheiham, A., \& Lawson, M. (2004). Demographic, familial and trait predictors of fruit and vegetable consumption by pre-school children. Public Health Nutr, 7(2), 295-302.

Coon, K.A., Goldberg, J., Rogers, B.L., \& Tucker, K.L. (2001). Relationships between use of television during meals and children's food consumption patterns. Pediatrics, 107(1), E7.

Darling, N., \& Steinberg, L. (1993). Parenting style as a context: an integrative model. Psychol Bull, $113,487-496$.

Davis, K.F., Parker, K.P., \& Montgomery, G.L. (2004). Sleep in infants and young children: Part one: normal sleep. $J$ Pediatr Health Care, 18(2), 65-71.

Davison, K.K., \& Birch, L.L. (2004). Predictors of fat stereotypes among 9-year-old girls and their parents. Obes Res, 12(1), 86-94.

De Bourdeaudhuij, I., \& Van Oost, P. (2000). Personal and family determinants of dietary behaviour in adolescents and their parents. Psychol Health, 15, 751-770.

De Bruijn, G.J., Kremers, S.P., de Vries, H., van Mechelen, W., \& Brug, J. (2007). Associations of social-environmental and individual-level factors with adolescent soft drink consumption: results from the SMILE study. Health Educ Res, 22(2), 227-237.

De Kroon, M.L., Renders, C.M., Van Wouwe, J.P., Van Buuren, S., \& Hirasing, R.A (2010). The Terneuzen birth cohort: BMI changes between 2 and 6 years correlate strongest with adult overweight. PLoS One, 5(2), e9155.

De Schipper, J.C., Tavecchio, L.W.C., \& Van IJzendoorn, M.H. (2007). Children's Attachment Relationships with Day Care Caregivers: Associations with Positive Caregiving and the Child's Temperament. Soc Dev, 17.

Dowda, M., Pate, R.R., Trost, S.G., Almeida, M.J., \& Sirard, J.R. (2004). Influences of preschool policies and practices on children's physical activity. J Commun Health, 29(3), 183-196.

DuRant, R.H., Baranowski, T., Johnson, M., \& Thompson, W.O. (1994). The relationship among television watching, physical activity, and body composition of young children. Pediatrics, 94(4 Pt 1), 449-455.

Dutman, A.E., Stafleu, A., Kruizinga, A., Brants, H.A., Westerterp, K.R., Kistemaker, C., Meuling, W.J., \& Goldbohm, R.A. (2010). Validation of an FFQ and options for data processing using the doubly labelled water method in children. Public Health Nutr, Epub August $16^{\text {th }} 2010$.

Ebbeling, C.B., Pawlak, D.B., \& Ludwig, D.S. (2002). Childhood obesity: public-health crisis, common sense cure. Lancet, 360(9331), 473-482.

Environmental Health Risks in European Birth Cohorts (ENRIECO) (2010). ENRIECO Database. 
Erlanson-Albertsson, C., \& Zetterstrom, R. (2005). The global obesity epidemic: snacking and obesity may start with free meals during infant feeding. Acta Paediatr, 94(11), 1523-1531.

Erlanson-Albertsson, C., \& Zetterstrom, R. (2006). Reply to letter by Aarts and Greiner. Acta Paediatr, 95(5), 624-625.

Eurostat. (2007). Task Force on Core Social Variables. Final Report. Luxembourg: European Communitees.

Eurostat. (2008). Childcare in the EU in 2006. A quarter of children aged less than three in formal childcare. And more than $80 \%$ of children from three to compulsory school age. Eurstat Press Office.

Ezendam, N.P., Evans, A.E., Stigler, M.H., Brug, J., \& Oenema, A (2010). Cognitive and home environmental predictors of change in sugar-sweetened beverage consumption among adolescents. Br J Nutr, 103(5), 768-774.

Faith, M.S., Berkowitz, R.I., Stallings, V.A., Kerns, J., Storey, M., \& Stunkard, A.J. (2004). Parental feeding attitudes and styles and child body mass index: prospective analysis of a geneenvironment interaction. Pediatrics, 114(4), e429-436.

Faith, M.S., \& Kerns, J. (2005). Infant and child feeding practices and childhood overweight: the role of restriction. Matern Child Nutr, 1(3), 164-168.

Faith, M.S., Scanlon, K.S., Birch, L.L., Francis, L.A., \& Sherry, B. (2004). Parent-child feeding strategies and their relationships to child eating and weight status. Obes Res, 12(11), 17111722 .

Farrow, C., \& Blissett, J. (2006). Breast-feeding, maternal feeding practices and mealtime negativity at one year. Appetite, 46(1), 49-56.

Ferreira, I., van der Horst, K., Wendel-Vos, W., Kremers, S., van Lenthe, F.J., \& Brug, J. (2007). Environmental correlates of physical activity in youth - a review and update. Obes Rev, 8(2), 129-154.

Field, A. (2005). Discovering Statistics Using SPSS. London, Thousand Oaks, New Delhi: Sage Publications.

Fisher, J.O., \& Birch, L.L. (1999). Restricting access to foods and children's eating. Appetite, 32(3), 405-419.

Fisher, J.O., Birch, L.L., Smiciklas-Wright, H., \& Picciano, M.F. (2000). Breast-feeding through the first year predicts maternal control in feeding and subsequent toddler energy intakes. $J \mathrm{Am}$ Diet Assoc, 100(6), 641-646.

Fisher, J.O., Rolls, B.J., \& Birch, L.L. (2003). Children's bite size and intake of an entree are greater with large portions than with age-appropriate or self-selected portions. Am J Clin Nutr, 77(5), 1164-1170.

Forestell, C.A., \& Mennella, J.A. (2007). Early determinants of fruit and vegetable acceptance. Pediatrics, 120(6), 1247-1254.

Fredriks, A.M., van Buuren, S., Wit, J.M., \& Verloove-Vanhorick, S.P. (2000). Body index measurements in 1996-7 compared with 1980. Arch Dis Child, 82(2), 107-112.

Friedman, S.L., \& Wachs, T.D. (1999). Measuring Environment Across the Life Span. Emerging Methods and Concepts. Washington: American Psychological Association.

Frisvold, D.E., \& Lumeng, J.C. (2007). Childhood Obesity and Exposure to Head Start Services: University of Michigan.

Gillman, M.W., Rifas-Shiman, S.L., Camargo, C.A. Jr., Berkey, C.S., Frazier, A.L., Rockett, H.R., Field, A.E., \& Colditz, G.A. (2001). Risk of overweight among adolescents who were breastfed as infants. J Amer Med Assoc, 285(19), 2461-2467.

Golan, M., \& Crow, S. (2004). Parents are key players in the prevention and treatment of weight-related problems. Nutr Rev, 62(1), 39-50.

Gordon, R. (1987). An operational classification of disease prevention. In J. A. Steinberg \& M. M. Silverman (Eds.), Preventing Mental Disorders. Rockville, MD: U.S. Department of Health and Human Services. 
Gubbels, J.S., Kremers, S.P., Stafleu, A., Dagnelie, P.C., de Vries, N.K., \& Thijs, C. (2010). Child-care environment and dietary intake of 2- and 3-year-old children. J Hum Nutr Diet, 23(1), 97101.

Gubbels, J.S., Kremers, S.P., Stafleu, A., Dagnelie, P.C., Goldbohm, R.A., de Vries, N.K., \& Thijs, C. (2009). Diet-related restrictive parenting practices. Impact on dietary intake of 2-year-old children and interactions with child characteristics. Appetite, 52(2), 423-429.

Gubbels, J.S., Kremers, S.P.J., Stafleu, A., Dagnelie, P.C., De Vries, N.K., van Buuren, S., \& Thijs, C. (2010). Child-care use and the association with body mass index and overweight in children from 7 months to 2 years of age. Int $J$ Obes, Epub May $25^{\text {th }} 2010$.

Halford, J.C., Boyland, E.J., Hughes, G., Oliveira, L.P., \& Dovey, T.M. (2007). Beyond-brand effect of television (TV) food advertisements/commercials on caloric intake and food choice of 5-7year-old children. Appetite, 49(1), 263-267.

Hannon, J.C., \& Brown, B.B. (2008). Increasing preschoolers' physical activity intensities: an activityfriendly preschool playground intervention. Prev Med, 46(6), 532-536.

Harder, T., Bergmann, R., Kallischnigg, G., \& Plagemann, A. (2005). Duration of breastfeeding and risk of overweight: a meta-analysis. Am J Epidemiol, 162(5), 397-403.

He, M., Piche, L., Beynon, C., \& Harris, S. (2010). Screen-related sedentary behaviors: children's and parents' attitudes, motivations, and practices. J Nutr Educ Behav, 42(1), 17-25.

Hediger, M.L., Overpeck, M.D., Kuczmarski, R.J., \& Ruan, W.J. (2001). Association between infant breastfeeding and overweight in young children. J Amer Med Assoc, 285(19), 2453-2460.

Hendy, H.M., \& Raudenbush, B. (2000). Effectiveness of teacher modeling to encourage food acceptance in preschool children. Appetite, 34(1), 61-76.

Hinkley, T., Crawford, D., Salmon, J., Okely, A.D., \& Hesketh, K. (2008). Preschool children and physical activity: a review of correlates. Am J Prev Med, 34(5), 435-441.

Hughes, S.O., Patrick, H., Power, T.G., Fisher, J.O., Anderson, C.B., \& Nicklas, T.A. (2007). The impact of child care providers' feeding on children's food consumption. J Dev Behav Pediatr, 28(2), 100-107.

Hupkens, C.L., Knibbe, R.A., Van Otterloo, A.H., \& Drop, M.J. (1998). Class differences in the food rules mothers impose on their children: a cross-national study. Soc Sci Med, 47(9), 13311339.

Huston, A.C., Chang, Y.E., \& Gennetian, L. (2002). Family and individual predictors of childcare use by low-income families in different policy contexts. Early Child Res Q, 17, 441-469.

Huus, K., Ludvigsson, J.F., Enskar, K., \& Ludvigsson, J. (2008). Exclusive breastfeeding of Swedish children and its possible influence on the development of obesity: a prospective cohort study. BMC Pediatr, 8, 42.

Johannsson, E., Arngrimsson, S.A., Thorsdottir, I., \& Sveinsson, T. (2006). Tracking of overweight from early childhood to adolescence in cohorts born 1988 and 1994: overweight in a high birth weight population. Int J Obes, 30(8), 1265-1271.

Johnson, S.L., \& Birch, L.L. (1994). Parents' and children's adiposity and eating style. Pediatrics, 94(5), 653-661.

Jouret, B., Ahluwalia, N., Dupuy, M., Cristini, C., Negre-Pages, L., Grandjean, H., \& Tauber, M. (2009). Prevention of overweight in preschool children: results of kindergarten-based interventions. Int J Obes, 33(10), 1075-1083.

Kelder, S.H., Perry, C.L., Klepp, K.I., \& Lytle, L.L. (1994). Longitudinal tracking of adolescent smoking, physical activity, and food choice behaviors. Am J Public Health, 84(7), 11211126.

Keller, K.L., Pietrobelli, A., Johnson, S.L., \& Faith, M.S. (2006). Maternal restriction of children's eating and encouragements to eat as the 'non-shared environment': a pilot study using the child feeding questionnaire. Int J Obes, 30(11), 1670-1675.

Kim, J., \& Peterson, K.E. (2008). Association of infant child care with infant feeding practices and weight gain among US infants. Arch Pediat Adol Med, 162(7), 627-633. 
Kimbro, R.T., Brooks-Gunn, J., \& McLanahan, S. (2007). Racial and ethnic differentials in overweight and obesity among 3-year-old children. Am J Public Health, 97(2), 298-305.

Knol, L.L., Haughton, B., \& Fitzhugh, E.C. (2005). Dietary patterns of young, low-income US children. $J$ Am Diet Assoc, 105(11), 1765-1773.

Koot, H. M., Van Den Oord, E.J., Verhulst, F.C., \& Boomsma, D.I. (1997). Behavioral and emotional problems in young preschoolers: cross-cultural testing of the validity of the Child Behavior Checklist/2-3. J Abnorm Child Psychol, 25(3), 183-196.

Kramer, M.S., Guo, T., Platt, R.W., Shapiro, S., Collet, J.P., Chalmers, B., Hodnett, E., Sevkovskaya, Z., Dzikovich, I., \& Vanilovich, I. (2002). Breastfeeding and infant growth: biology or bias? Pediatrics, $110(2 \mathrm{Pt} 1), 343-347$

Krebs, N.F., \& Jacobson, M.S. (2003). Prevention of pediatric overweight and obesity. Pediatrics, $112(2), 424-430$.

Kremers, S.P. (2010). Theory and practice in the study of influences on energy balance-related behaviors. Patient Educ Couns.

Kremers, S.P., Brug, J., de Vries, H., \& Engels, R.C. (2003). Parenting style and adolescent fruit consumption. Appetite, 41(1), 43-50.

Kremers, S.P., de Bruijn, G.J., Droomers, M., van Lenthe, F., \& Brug, J. (2007). Moderators of environmental intervention effects on diet and activity in youth. Am J Prev Med, 32(2), 163172.

Kremers, S.P., de Bruijn, G.J., Visscher, T.L., van Mechelen, W., de Vries, N.K., \& Brug, J. (2006). Environmental influences on energy balance-related behaviors: a dual-process view. Int $J$ Behav Nutr Phys Act, 3, 9.

Kremers, S.P., van der Horst, K., \& Brug, J. (2007). Adolescent screen-viewing behaviour is associated with consumption of sugar-sweetened beverages: the role of habit strength and perceived parental norms. Appetite, 48(3), 345-350.

Kremers, S.P., Visscher, T.L., Brug, J., Chin, A.P.M.J., Schouten, E.G., Schuit, A.J., Seidell, J.C., Van Baak, M.A., Van Mechelen, W., Kemper, H.C., Kok, F.J., Saris, W.H., \& Kromhout, D. (2005). Netherlands research programme weight gain prevention (NHF-NRG): rationale, objectives and strategies. Eur J Clin Nutr, 59(4), 498-507.

Kremers, S.P., Visscher, T.L., Seidell, J.C., van Mechelen, W., \& Brug, J. (2005). Cognitive determinants of energy balance-related behaviours: measurement issues. Sports Med, 35(11), 923-933.

Kremers, S.P.J., De Bruin, G.J., Schaalma, H., \& Brug, J. (2004). Clustering of energy balance-related behaviours and their intrapersonal determinants. Psychol Health, 19(5), 595-606.

Kremers, S.P.J., Schaalma, H., Meertens, R.M., van Mechelen, W., \& Kok, G.J. (2008). Systematic Promotion of Physical Activity. In N. Armstrong \& W. van Mechelen (Eds.), Peadiatric Exercise Science and Medicine. Oxford: Oxford University Press.

Kroller, K., \& Warschburger, P. (2009). Maternal feeding strategies and child's food intake: considering weight and demographic influences using structural equation modeling. Int $J$ Behav Nutr Phys Act, 6, 78.

Kummeling, I., Thijs, C., Penders, J., Snijders, B.E., Stelma, F., Reimerink, J., Koopmans, M., Dagnelie, P.C., Huber, M., Jansen, M.C., de Bie, R., \& van den Brandt, P.A. (2005). Etiology of atopy in infancy: the KOALA Birth Cohort Study. Pediatr Allergy Immu, 16(8), 679-684.

Lande, B., Andersen, L.F., Veierod, M.B., Baerug, A., Johansson, L., Trygg, K.U., \& Bjorneboe, G.E. (2004). Breast-feeding at 12 months of age and dietary habits among breast-fed and nonbreast-fed infants. Public Health Nutr, 7(4), 495-503.

Landis, J.R., \& Koch, G.G. (1977). The measurement of observer agreement for categorical data. Biometrics, 33(1), 159-174.

Latner, J.D., \& Stunkard, A.J. (2003). Getting worse: the stigmatization of obese children. Obes Res, 11(3), 452-456.

Lewin, K. (1935). A dynamic theory of personality. New York: McGraw-Hill. 
Liem, D.G., Mars, M., \& De Graaf, C. (2004). Sweet preferences and sugar consumption of 4- and 5year-old children: role of parents. Appetite, 43(3), 235-245.

Lioret, S., Touvier, M., Lafay, L., Volatier, J.L., \& Maire, B. (2008). Dietary and physical activity patterns in French children are related to overweight and socioeconomic status. $J$ Nutr, 138(1), 101-107.

Lumeng, J.C., Gannon, K., Appugliese, D., Cabral, H.J., \& Zuckerman, B. (2005). Preschool child care and risk of overweight in 6- to 12-year-old children. Int J Obes, 29(1), 60-66.

Maher, E.J., Li, G., Carter, L., \& Johnson, D.B. (2008). Preschool child care participation and obesity at the start of kindergarten. Pediatrics, 122(2), 322-330.

Marchini, G., Persson, B., Berggren, V., \& Hagenas, L. (1998). Hunger behaviour contributes to early nutritional homeostasis. Acta Paediatr, 87(6), 671-675.

Marshall, S.J., Biddle, S.J., Gorely, T., Cameron, N., \& Murdey, I. (2004). Relationships between media use, body fatness and physical activity in children and youth: a meta-analysis. Int $J$ Obes Relat Metab Disord, 28(10), 1238-1246.

Matheson, D.M., Robinson, T.N., Varady, A., \& Killen, J.D. (2006). Do Mexican-American mothers' food-related parenting practices influence their children's weight and dietary intake? $\mathrm{J} \mathrm{Am}$ Diet Assoc, 106(11), 1861-1865.

McCarthy, H.D. (2006). Body fat measurements in children as predictors for the metabolic syndrome: focus on waist circumference. P Nutr Soc, 65, 385-392.

McWilliams, C., Ball, S.C., Benjamin, S.E., Hales, D., Vaughn, A., \& Ward, D.S. (2009). Best-practice guidelines for physical activity at child care. Pediatrics, 124(6), 1650-1659.

Mennella, J.A., Jagnow, C.P., \& Beauchamp, G.K. (2001). Prenatal and postnatal flavor learning by human infants. Pediatrics, 107(6), E88.

Moens, E., Braet, C., \& Soetens, B. (2007). Observation of family functioning at mealtime: a comparison between families of children with and without overweight. J Pediatr Psychol, $32(1), 52-63$.

Monitor Jeugdgezondheid. (2009). Standaard Vraagstelling Bewegen (Standard Questionnaire Physical Activity): GGD Nederland, Rijksinstituut voor Volksgezondheid en Milieu (RIVM) and Actiz organisatie van zorgondernemers.

Montgomery, C., Jackson, D.M., Kelly, L.A., \& Reilly, J.J. (2006). Parental feeding style, energy intake and weight status in young Scottish children. Br J Nutr, 96(6), 1149-1153.

Muller, M.J., Mast, M., Asbeck, I., Langnase, K., \& Grund, A. (2001). Prevention of obesity--is it possible? Obes Rev, 2(1), 15-28.

Musher-Eizenman, D., \& Holub, S. (2007). Comprehensive Feeding Practices Questionnaire: validation of a new measure of parental feeding practices. J Pediatr Psychol, 32(8), 960-972.

Musher-Eizenman, D.R., \& Holub, S.C. (2006). Children's Eating in the Absence of Hunger: The Role of Restrictive Feeding Practices. In R. K. Flamenbaum (Ed.), Childhood Obesity and Health Research (pp. 135-156). New York: Nova Science Publishers, Inc.

Nationaal Kompas Volksgezondheid. (2008). Jeugdgezondheidszorg samengevast. In: Volksgezondheid Toekomst Verkenning. Bilthoven: RIVM.

Nelson, M.C., Gordon-Larsen, P., Adair, L.S., \& Popkin, B.M. (2005). Adolescent physical activity and sedentary behavior: patterning and long-term maintenance. Am J Prev Med, 28(3), 259-266.

Neumark-Sztainer, D., Story, M., Toporoff, E., Himes, J.H., Resnick, M.D., \& Blum, R.W. (1997). Covariations of eating behaviors with other health-related behaviors among adolescents. $J$ Adolesc Health, 20(6), 450-458.

Neutzling, M.B., Hallal, P.R., Araujo, C.L., Horta, B.L., Vieira Mde, F., Menezes, A.M., \& Victora, C.G. (2009). Infant feeding and obesity at 11 years: prospective birth cohort study. Int $J$ Pediatr Obes, 4(3), 143-149.

Northstone, K., \& Emmett, P. (2005). Multivariate analysis of diet in children at four and seven years of age and associations with socio-demographic characteristics. Eur J Clin Nutr, 59(6), 751760.

Nunnally, J.C., \& Bernstein, I.H. (1994). Psychometric Theory. New York: McGraw and Hill. 
Owen, C.G., Martin, R.M., Whincup, P.H., Smith, G.D., \& Cook, D.G. (2005). Effect of infant feeding on the risk of obesity across the life course: a quantitative review of published evidence. Pediatrics, 115(5), 1367-1377.

Padget, A., \& Briley, M.E. (2005). Dietary intakes at child-care centers in central Texas fail to meet Food Guide Pyramid recommendations. J Am Diet Assoc, 105(5), 790-793.

Pate, R.R., McIver, K., Dowda, M., Brown, W.H., \& Addy, C. (2008). Directly observed physical activity levels in preschool children. $J$ School Health, 78(8), 438-444.

Patterson, E., Warnberg, J., Kearney, J., \& Sjostrom, M. (2009). The tracking of dietary intakes of children and adolescents in Sweden over six years: the European Youth Heart Study. Int $J$ Behav Nutr Phys Act, 6, 91.

Pollit, D.F., \& Beck, C.T. (2004). Nursing Reseach. Principles and Methods. (7th ed.). Philadelphia: Lippincott Williams \& Wilkins.

Portegijs, C., Hermans, B., \& Lalta, V. (2006). Emancipatiemonitor 2006. Veranderingen in leefsituatie en levensloop. Den Haag: Sociaal en Cultureel Planbureau \& Centraal Bureau voor de Statistiek.

Powers, S.W., Chamberlin, L.A., van Schaick, K.B., Sherman, S.N., \& Whitaker, R.C. (2006). Maternal feeding strategies, child eating behaviors, and child BMI in low-income African-American preschoolers. Obesity (Silver Spring), 14(11), 2026-2033.

Puhl, R.M., \& Schwartz, M.B. (2003). If you are good you can have a cookie: How memories of childhood food rules link to adult eating behaviors. Eat Behav, 4(3), 283-293.

Rapp, K., Schick, K.H., Bode, H., \& Weiland, S.K. (2005). Type of kindergarten and other potential determinants of overweight in pre-school children. Public Health Nutr, 8(6), 642-649.

Reilly, J.J., Jackson, D.M., Montgomery, C., Kelly, L.A., Slater, C., Grant, S., \& Paton, J.Y. (2004). Total energy expenditure and physical activity in young Scottish children: mixed longitudinal study. Lancet, 363(9404), 211-212.

Rennie, K.L., Johnson, L., \& Jebb, S.A. (2005). Behavioural determinants of obesity. Best Pract Res Clin Endocrinol Metab, 19(3), 343-358.

Rhee, K. (2008). Childhood Overweight and the Relationship between Parent Behaviors, Parenting style, and family functioning. Ann Am Acad Polit SS, 615(11).

Rhee, K.E., Lumeng, J.C., Appugliese, D.P., Kaciroti, N., \& Bradley, R.H. (2006). Parenting styles and overweight status in first grade. Pediatrics, 117(6), 2047-2054.

Ridgers, N.D., Stratton, G., Fairclough, S.J., \& Twisk, J.W. (2007a). Children's physical activity levels during school recess: a quasi-experimental intervention study. Int J Behav Nutr Phys Act, 4, 19.

Ridgers, N.D., Stratton, G., Fairclough, S.J., \& Twisk, J.W. (2007b). Long-term effects of a playground markings and physical structures on children's recess physical activity levels. Prev Med, 44(5), 393-397.

Robinson, T.N., Hammer, L.D., Killen, J.D., Kraemer, H.C., Wilson, D.M., Hayward, C., \& Taylor, C.B. (1993). Does television viewing increase obesity and reduce physical activity? Crosssectional and longitudinal analyses among adolescent girls. Pediatrics, 91(2), 273-280.

Rosenberg, D.E., Sallis, J.F., Kerr, J., Maher, J., Norman, G.J., Durant, N., Harris, S.K., \& Saelens, B.E. (2010). Brief scales to assess physical activity and sedentary equipment in the home. Int J Behav Nutr Phys Act, 7, 10.

Sallis, J.F., Prochaska, J.J., \& Taylor, W.C. (2000). A review of correlates of physical activity of children and adolescents. Med Sci Sports Exerc, 32(5), 963-975.

Salmon, J., Timperio, A., Telford, A., Carver, A., \& Crawford, D. (2005). Association of family environment with children's television viewing and with low level of physical activity. Obes Res, 13(11), 1939-1951.

Savage, J.S., Fisher, J.O., \& Birch, L.L. (2007). Parental influence on eating behavior: conception to adolescence. $J$ Law Med Ethics, 35(1), 22-34. 
Schack-Nielsen, L., Sorensen, T., Mortensen, E.L., \& Michaelsen, K.F. (2010). Late introduction of complementary feeding, rather than duration of breastfeeding, may protect against adult overweight. Am J Clin Nutr, 91(3), 619-627.

Scholtens, S., Brunekreef, B., Smit, H.A., Gast, G.C., Hoekstra, M.O., de Jongste, J.C., Postma, D.S., Gerritsen, J., Seidell, J.C., \& Wijga, A.H. (2008). Do differences in childhood diet explain the reduced overweight risk in breastfed children? Obesity (Silver Spring), 16(11), 24982503.

Scholtens, S., Brunekreef, B., Visscher, T.L., Smit, H.A., Kerkhof, M., de Jongste, J.C., Gerritsen, J., \& Wijga, A.H. (2007). Reported versus measured body weight and height of 4-year-old children and the prevalence of overweight. Eur J Public Health, 17(4), 369-374.

Schuit, A.J., van Loon, A.J., Tijhuis, M., \& Ocke, M. (2002). Clustering of lifestyle risk factors in a general adult population. Prev Med, 35(3), 219-224.

Singer, M.R., Moore, L.L., Garrahie, E.J., \& Ellison, R.C. (1995). The tracking of nutrient intake in young children: the Framingham Children's Study. Am J Public Health, 85(12), 1673-1677.

Singh, A.S., Mulder, C., Twisk, J.W., van Mechelen, W., \& Chinapaw, M.J. (2008). Tracking of childhood overweight into adulthood: a systematic review of the literature. Obes Rev, 9(5), 474-488.

Sleddens, E.F., Kremers, S.P., De Vries, N.K., \& Thijs, C. (2010). Relationship between parental feeding styles and eating behaviours of Dutch children aged 6-7. Appetite, 54(1), 30-36.

Spence, J.C., \& Lee, R.E. (2003). Toward a comprehensive model of physical activity. Psychol Sport Exerc, 4, 7-24.

Spurrier, N.J., Magarey, A.A., Golley, R., Curnow, F., \& Sawyer, M.G. (2008). Relationships between the home environment and physical activity and dietary patterns of preschool children: a cross-sectional study. Int J Behav Nutr Phys Act, 5, 31 .

Stang, J., Rehorst, J., \& Golicic, M. (2004). Parental feeding practices and risk of childhood overweight in girls: implications for dietetics practice. J Am Diet Assoc, 104(7), 1076-1079.

Stichting Nederlands Voedingsstoffenbestand. (2001). Nederlands voedingsstoffenbestand (NEVO) 2001 (Netherlands Food Consumption Table 2001). Den Haag, the Netherlands: Voedingscentrum.

Stichting Nederlands Voedingsstoffenbestand (2006). Nederlands voedingsstoffenbestand (NEVO) 2006. (Netherlands Food Composition Table 2006). Den Haag, the Netherlands: Voedingscentrum.

Story, M., Kaphingst, K.M., \& French, S. (2006). The role of child care settings in obesity prevention. Future Child, 16(1), 143-168.

Stratton, G. (2000). Promoting children's physical activity in primary school: an intervention study using playground markings. Ergonomics, 43(10), 1538-1546.

Striegel-Moore, R.H., Thompson, D., Affenito, S.G., Franko, D.L., Obarzanek, E., Barton, B.A., Schreiber, G.B., Daniels, S.R., Schmidt, M., \& Crawford, P.B. (2006). Correlates of beverage intake in adolescent girls: the National Heart, Lung, and Blood Institute Growth and Health Study. J Pediatr, 148(2), 183-187.

Swinburn, B., Egger, G., \& Raza, F. (1999). Dissecting obesogenic environments: the development and application of a framework for identifying and prioritizing environmental interventions for obesity. Prev Med, 29(6 Pt 1), 563-570.

Tavecchio, L., IJzendoorn, R. v., \& Stams, G.J. (1996). Onderzoek: kinderopvang en thuis twee gescheiden werelden. Kinderopvang, 12, 24-27.

Taveras, E.M., Rifas-Shiman, S.L., Scanlon, K.S., Grummer-Strawn, L.M., Sherry, B., \& Gillman, M.W. (2006). To what extent is the protective effect of breastfeeding on future overweight explained by decreased maternal feeding restriction? Pediatrics, 118(6), 2341-2348.

Taveras, E.M., Scanlon, K.S., Birch, L., Rifas-Shiman, S.L., Rich-Edwards, J.W., \& Gillman, M.W. (2004). Association of breastfeeding with maternal control of infant feeding at age 1 year. Pediatrics, 114(5), e577-583. 
Temple, J.L., Giacomelli, A.M., Kent, K.M., Roemmich, J.N., \& Epstein, L.H. (2007). Television watching increases motivated responding for food and energy intake in children. Am J Clin Nutr, 85(2), 355-361.

Tiggemann, M., \& Lowes, J. (2002). Predictors of maternal control over children's eating behaviour. Appetite, 39(1), 1-7.

Timperio, A., Salmon, J., Ball, K., Baur, L.A., Telford, A., Jackson, M., Salmon, L., \& Crawford, D. (2008). Family physical activity and sedentary environments and weight change in children. Int J Pediatr Obes, 1-8.

Toschke, A.M., Vignerova, J., Lhotska, L., Osancova, K., Koletzko, B., \& Von Kries, R. (2002). Overweight and obesity in 6- to 14-year-old Czech children in 1991: protective effect of breast-feeding. J Pediatr, 141(6), 764-769.

United Nations Educational Scientific and Cultural Organization. (1997). International Standard Classification of Education UNESCO.

Utter, J., Neumark-Sztainer, D., Jeffery, R., \& Story, M. (2003). Couch potatoes or french fries: are sedentary behaviors associated with body mass index, physical activity, and dietary behaviors among adolescents? J Am Diet Assoc, 103(10), 1298-1305.

Van den Hurk, K., van Dommelen, P., van Buuren, S., Verkerk, P.H., \& Hirasing, R.A. (2007). Prevalence of overweight and obesity in the Netherlands in 2003 compared to 1980 and 1997. Arch Dis Child, 92(11), 992-995.

Van der Horst, K., Kremers, S., Ferreira, I., Singh, A., Oenema, A., \& Brug, J. (2007). Perceived parenting style and practices and the consumption of sugar-sweetened beverages by adolescents. Health Educ Res, 22(2), 295-304.

Van der Horst, K., Oenema, A., Ferreira, I., Wendel-Vos, W., Giskes, K., van Lenthe, F., \& Brug, J. (2006). A systematic review of environmental correlates of obesity-related dietary behaviors in youth. Health Educ Res, 22(2), 203-226.

Van IJzendoorn, M.H., Tavecchio, L.W., Stams, G.J., Verhoeven, M.J., \& Reiling, E J. (1998a). Quality of center day care and attunement between parents and caregivers: center day care in cross-national perspective. J Genet Psychol, 159(4), 437-454.

Van IJzendoorn, M.H., Tavecchio, L.W.C., Stams, G.J., Verhoeven, M., \& Reiling, E. (1998b). Attunement Between Parents and Professional Caregivers: A Comparison of Childrearing Attitudes in Different Child-care Settings. J Marriage Fam, 60, 771-781.

Vereecken, C.A., Keukelier, E., \& Maes, L. (2004). Influence of mother's educational level on food parenting practices and food habits of young children. Appetite, 43(1), 93-103.

Voedingscentrum. (2007). Voeding van zuigelingen en peuters. Uitgangspunten voor de voedingsadvisering voor kinderen van 0-4 jaar. Den Haag, the Netherlands: Voedingscentrum.

Voedingscentrum. (2008). Borstvoeding. De beste start. Den Haag, the Netherlands: Voedingscentrum.

Wachs, T.D. (1992). The nature of nurture. Newbury Park, CA: Sage.

Ward, D., Hales, D., Haverly, K., Marks, J., Benjamin, S., Ball, S., \& Trost, S. (2008). An instrument to assess the obesogenic environment of child care centers. Am J Health Behav, 32(4), 380-386.

Wardle, J., \& Carnell, S. (2007). Parental feeding practices and children's weight. Acta Paediatr Suppl, 96(454), 5-11.

WHO/FAO Expert Consultation. (2003). Joint WHO/FAO Expert Consultation: Diet, nutrition and the prevention of chronic diseases. Geneva: WHO.

World Health Organization. (2004). Obesity: preventing and managing the global epidemic: report of a WHO consultation. Geneva: WHO.

World Health Organization. (2006). Obesity and Overweight. Geneva: WHO.

World Health Organization. (2009). Infant and young child feeding: Model chapter for textbooks for medical students and allied health professionals. Geneva: WHO.

World Health Organization. (2010). Childhood overweight and obesity on the rise. WHO. Retrieved, from the World Wide Web: http://www.who.int/dietphysicalactivity/childhood/en/ 
Wronka, I., \& Pawlinska-Chmara, R. (2007). Childcare, height and BMI among female Polish university students, 2005. Econ Hum Biol, 5(3), 435-442.

Zabinski, M.F., Daly, T., Norman, G.J., Rupp, J.W., Calfas, K.J., Sallis, J.F., \& Patrick, K. (2006). Psychosocial correlates of fruit, vegetable, and dietary fat intake among adolescent boys and girls. J Am Diet Assoc, 106(6), 814-821.

Zask, A., van Beurden, E., Barnett, L., Brooks, L.O., \& Dietrich, U.C. (2001). Active school playgrounds-myth or reality? Results of the "move it groove it" project. Prev Med, 33(5), 402-408.

Zaslow, M.J., Oldham, E., \& Moore, K.A. (1998). Welfare Families' Use of Early Childhood Care and Education Programs, and Implications for Their Children's Development. Early Child Res $Q$, 13(4), 535-563. 

Summary 
The prevalence of childhood overweight and obesity has reached epidemic proportions (World Health Organization, 2004), also in the Netherlands (Van den Hurk et al., 2007). The pre-school age (2-6 years) has been indicated as the most critical growth period for adult overweight (De Kroon et al., 2010). Treatment has been shown to have poor results for children and adolescents already suffering from obesity (Ben-Sefer et al., 2009). Early prevention is therefore essential to effectively counteract the obesity epidemic (Kremers, Visscher, Brug et al., 2005).

The aim of the dissertation was to examine the direct and interactive effects of specific features of micro environments on young children's energy balancerelated behaviours (EBRBs, i.e., dietary intake and physical activity) and weight status. The micro environments that were examined were those in which young children spend most of their time, i.e., the home environment and the child-care environment. The ecological perspective of environmental influences on behaviour (Friedman \& Wachs, 1999; Spence \& Lee, 2003; Wachs, 1992) is used as a theoretical framework for the current dissertation. This ecological perspective assumes that physical environmental factors interact with the actors that operate within this setting (i.e., the social environment) in influencing behaviour. In addition, the influence of the environment on behaviour can be moderated by several individual factors (Kremers et al., 2006; Kremers et al., 2008). The current dissertation is based on data of the KOALA Birth Cohort Study (Kummeling et al., 2005) and on observations in child-care centres.

\section{The home environment}

The first part of this dissertation discusses the role of the home environment in the development of young children's energy balance-related habits (i.e., dietary intake and physical activity habits), as well as its role in the development of their weight status. Particularly, the role of parents in this respect is examined. Chapter 2 addresses the influence of breast-feeding duration and feeding pattern on weight development up to the age of 4 years. The study confirms the earlier reported protective effect of breast-feeding against overweight development (Arenz et al., 2004; Harder et al., 2005; Owen et al., 2005). In addition, it shows that the feeding pattern (i.e., feeding on demand of the child vs. on a fixed time schedule) is not a plausible mechanism through which this protective effect operates. Finally, an inverse association between breast-feeding and snacking is found, which might be a possible mechanism for the protective effect of breast-feeding on overweight at a later age.

Chapter 3 examines clustering or co-occurrence of dietary intake and physical activity, and showed that clustering of EBRBs is already present in 2-yearolds: healthy intake behaviours, such as fruit, vegetable and brown bread intake were found to cluster. In addition, a second cluster was found, in which sedentary behaviour (i.e., computer use and television viewing) is associated with unhealthy 
intake behaviour (i.e., snack and soft drink intake). Moreover, Chapter 3 shows that these clusters are related to maternal characteristics: children of obese and lower educated mothers more often showed behaviour within the unhealthy 'sedentarysnacking' cluster, and scored less well on the healthy cluster.

Chapters 4 (regarding 2-year-olds) and 5 (regarding 5- to 7-year-olds) describe the influence of energy balance-related parenting practices on children's dietary intake, physical activity and weight development. Restrictive parenting practices were shown to have a desirable impact on 2-year-olds' dietary intake. Monitoring a child's dietary intake and stimulation to eat healthy were both associated with desirable effects on 5-year-olds' dietary intake. Restriction of sedentary time was found to be associated with increased sedentary behaviour and decreased physical activity at age 5 , while stimulation to be active was found to have desirable effects at this age. In line with this finding, BMI development between ages 5 and 7 was positively associated with restriction of sedentary time, and negatively associated with stimulation to eat healthy.

Both Chapter 4 and Chapter 5 showed that the effects of the parenting practices were moderated by child-characteristics. Restrictive parenting practices were found to have less desirable effects, or even undesirable effects on children that are in some way deviant from a 'normal' child (in terms of weight, eating style or general behavioural style), and it would perhaps be wise to advise parents of such children against the use of such restrictive practices. Parents should better use a positive approach in their energy balance-related parenting, as stimulating healthy eating was found to be beneficial, specially for deviant children.

\section{The child-care environment}

The second part of the dissertation describes the influence child-care environments on children's EBRBs and weight status. Chapter 6 shows, in line with previous studies (Benjamin et al., 2009; Maher et al., 2008), a small but positive influence of child-care use on overweight development, indicating an increased risk of overweight in children who attend child-care compared to those that do not attend child-care. No information regarding specific environmental features at the childcare centres was known from this study and previous studies. This is therefore further examined in Chapters 7 and 8.

Chapter 7 shows that children at child-care ate more when staff ate together with them, as well as when a family serving style was used (in which the child can self-serve food). Staff discussing healthy foods with the children was found to be associated with a higher fibre intake. Chapter 8 shows that both the social and the physical child-care environment were significantly related to children's physical activity at child-care. Moreover, interactions were found between the social and the physical environment, as well as between the environment and characteristics of the child (i.e., age, gender). 
Chapter 9 discusses the practical and theoretical implications of the studies described above. It was concluded that there is a need to apply an energy balance approach in the prevention and examination of determinants of overweight. In addition, the home and child-care environment were identified as important settings for environmental interventions preventing early childhood overweight. Examples of specific features to be addressed in those environments are parental and childcare staff practices, and the availability of opportunities to be active or to consume certain foods. Furthermore, several possible target groups for interventions were identified, including parents who are overweight or obese themselves, and have long working hours. Finally, it was discussed that due to the interaction between the physical environment, the social environment, and child characteristics, as described above, environmental influences depend on the context and on characteristics of the specific child performing the behaviour. Future research should aim at translating research from the home setting to the child-care setting and at examining the interaction between home and the child-care setting, i.e., the influence of the meso system of children's EBRBs. 
Samenvatting 
De prevalentie van overgewicht en obesitas bij kinderen neemt in snel tempo en in sterke mate toe (World Health Organization, 2004), ook in Nederland (Van den Hurk et al., 2007). De leeftijd voordat kinderen naar school gaan (2-6 jaar) is aangeduid als de meest belangrijke groeiperiode voor de ontwikkeling van toekomstig overgewicht (De Kroon et al., 2010). Behandelingen laten vaak slechte resultaten zien bij kinderen en adolescenten die al te dik zijn (Ben-Sefer et al., 2009). Vroege preventie is daarom essentieel om effectief de obesitas epidemie tegen te kunnen gaan (Kremers, Visscher, Brug et al., 2005).

Het doel van het proefschrift was om de effecten van specifieke kenmerken in micro-omgevingen van jonge kinderen te onderzoeken op voedingsinname, fysieke activiteit en gewichtsstatus van jonge kinderen. De micro-omgevingen die onderzocht zijn, zijn omgevingen waarin jonge kinderen de meeste tijd doorbrengen, namelijk de thuisomgeving en de kinderdagverblijfomgeving. Het ecologische perspectief op de invloed van de omgeving op gedrag (Friedman \& Wachs, 1999; Spence \& Lee, 2003; Wachs, 1992) is gebruikt als theoretisch kader voor dit proefschrift. Dit ecologisch perspectief veronderstelt dat in de beïnvloeding van gedrag, de fysieke omgeving interacteert met de actoren die handelen in deze setting (d.w.z. de sociale omgeving). Daarnaast kan de invloed van de omgeving op gedrag gemodereerd worden door verscheidene individuele factoren (Kremers et al., 2006; Kremers et al., 2008). Het proefschrift is gebaseerd op data van het KOALA Geboortecohort Onderzoek (Kummeling et al., 2005) en op observaties in kinderdagverblijven.

\section{De thuisomgeving}

Het eerste deel van dit proefschrift gaat over de rol van de thuisomgeving in de ontwikkeling van voedingsgewoonten en bewegingsgewoonten van jonge kinderen. Daarnaast is er gekeken naar de invloed van de thuisomgeving op de ontwikkeling van overgewicht. Met name de rol van de ouders hierin is onderzocht. Hoofdstuk 2 behandelt de invloed van borstvoeding en het voedingsschema op de ontwikkeling van het gewicht van het kind tot en met een leeftijd van 4 jaar. De studie bevestigde het al eerder gerapporteerde beschermende effect van borstvoeding tegen de ontwikkeling van overgewicht (Arenz et al., 2004; Harder et al., 2005; Owen et al., 2005). Daarnaast liet de studie zien dat het voedingsschema (d.w.z. voeden op verzoek van het kind, of voeden volgens een vast tijdsschema) geen plausibel mechanisme is voor de verklaring van het beschermende effect van borstvoeding. Tot slot toonde het onderzoek een omgekeerde relatie tussen borstvoeding en snackconsumptie (zowel zoete als hartige snacks) door kinderen. Dit komt overeen met eerder onderzoek (Scholtens et al., 2008), en is wellicht een mechanisme voor de verklaring van het beschermende effect van borstvoeding tegen overgewicht op latere leeftijd. 
In Hoofdstuk 3 is clustering, of het samengaan, van voedingsinname en fysieke activiteit onderzocht. De studie laat zien dat clustering van voeding en fysieke activiteit al plaatsvindt bij 2-jarigen: gezonde voedingsgedragingen, zoals het eten van fruit, groenten en bruin brood, bleken vaak samen te gaan. Daarnaast werd een tweede cluster gevonden, waarin sedentair gedrag (computergebruik en televisiekijken) samenging met ongezonde voedingsgedragingen (snack- en frisdrankconsumptie). Bovendien laat Hoofdstuk 3 zien dat deze clusters gerelateerd zijn aan kenmerken van de moeder: kinderen van obese of lager opgeleide moeders vertoonden vaker gedrag in het ongezonde 'sedentaire-snack' cluster, en scoorden minder goed op het gezonde cluster.

Hoofdstukken 4 (over 2-jarigen) en 5 (over 5- tot 7-jarigen) beschrijven de invloed van energiebalans-gerelateerde ouderschapspraktijken op de voedingsinname, fysieke activiteit en gewichtsontwikkeling van kinderen. Restrictieve (verbiedende en beperkende) ouderschapspraktijken hadden een gewenst effect op de voedingsinname van 2-jarigen. Het in de gaten houden (monitoren) van wat het kind eet, en het stimuleren van gezonde voedingsinname, waren beiden geassocieerd met gewenste effecten op de voedingsinname van 5jarigen. Het verbieden of beperken van sedentaire gedragingen was echter geassocieerd met meer sedentair gedrag, en minder fysieke activiteit op 5-jarige leeftijd. Het stimuleren van fysieke activiteit had wel gewenste effecten op gedrag op deze leeftijd. In het verlengde van bovenstaande bevindingen vonden we dat BMI-ontwikkeling tussen 5 en 7 jaar positief geassocieerd was met het verbieden van sedentair gedrag, en negatief geassocieerd was met het stimuleren van gezonde voedingsinname.

Zowel in Hoofdstuk 4 als in Hoofdstuk 5 werd gevonden dat de effecten van de ouderschapspraktijken gemodereerd werden door kenmerken van het kind. Verbiedende ouderschapspraktijken hadden minder gewenste of zelfs ongewenste effecten op kinderen die op bepaalde manieren afwijken van een 'normaal' kind (in termen van gewicht, eetstijl en algemene gedragsstijl). Het zou goed zijn ouders van zulke 'deviante' kinderen af te raden om restrictieve praktijken te gebruiken. In plaats daarvan zouden ouders beter een positieve aanpak kunnen hanteren met betrekking tot hun energiebalans-gerelateerde ouderschapspraktijken. Het stimuleren van gezonde voedingsinname had namelijk gunstige effecten, in het bijzonder voor 'deviante' kinderen.

\section{De kinderdagverblijfomgeving}

Het tweede deel van het proefschrift beschrijft de invloed van de kinderdagverblijfomgeving op voedingsinname, fysieke activiteit en gewichtsstatus van kinderen. Hoofdstuk 6 laat een klein maar positief effect van kinderdagverblijfgebruik op ontwikkeling van overgewicht zien, wat betekent dat kinderen die naar het kinderdagverblijf gaan een groter risico op overgewicht 
hebben dan kinderen die niet naar het kinderdagverblijf gaan. Dit is in overeenstemming met eerdere studies (Benjamin et al., 2009; Maher et al., 2008). Er was geen informatie bekend over omgevingskenmerken binnen de kinderdagverblijven in deze en eerdere studies. In Hoofdstukken 7 en 8 is daarom daaraan meer aandacht besteed.

Hoofdstuk 7 laat zien dat kinderen meer eten op het kinderdagverblijf als de kinderdagverblijfmedewerkers samen met hen eten, en ook meer eten als ze zelf het eten mogen pakken (een zogenaamde 'family serving style'). Als de medewerkers met de kinderen praatten over gezonde voeding, dan aten de kinderen meer voedingsvezels. Hoofdstuk 8 laat zien dat zowel de sociale als de fysieke kinderdagverblijfomgeving gerelateerd is aan de fysieke activiteit van kinderen op het kinderdagverblijf. Bovendien werden er interacties gevonden tussen de sociale en fysieke omgeving, en tussen de omgeving en kenmerken van het kind (d.w.z. leeftijd, geslacht).

Hoofdstuk 9 behandelt de praktische en theoretische implicaties van de hierboven beschreven studies. Er kan geconcludeerd worden dat preventie van overgewicht, en ook onderzoek naar determinanten van overgewicht, zich gelijktijdig en gecombineerd zouden moeten richten op zowel voeding als beweging. Verder konden we de thuisomgeving en kinderdagverblijfomgeving aanwijzen als belangrijke omgevingen voor mogelijke interventies ter preventie van overgewicht bij kinderen. Voorbeelden van specifieke aspecten waarop zulke interventies zich zouden kunnen richten in die settings, zijn de opvoedingspraktijken van ouders en kinderdagverblijfmedewerkers, en de beschikbaarheid van mogelijkheden om actief te zijn of bepaalde voedingsmiddelen te consumeren. Daarnaast konden we verschillende mogelijke doelgroepen voor interventies identificeren, waaronder ouders die zelf overgewicht hebben of obees zijn, en ouders die veel buitenshuis werken. Ten slotte is bediscussieerd dat door de interactie tussen de fysieke omgeving, de sociale omgeving en kenmerken van het kind, omgevingsinvloeden afhankelijk zijn van de context en de kenmerken van het kind dat het gedrag vertoont. Toekomstig onderzoek zou zich moeten richten op het vertalen van onderzoek in de thuisomgeving naar de kinderdagverblijfomgeving. Bovendien kan onderzoek zich richten op de interactie tussen thuis en het kinderdagverblijf, oftewel de invloed van het meso-systeem op voeding en beweging bij kinderen. 


\section{Dankwoord}

Graag wil ik iedereen bedanken die op enige wijze heeft bijgedragen aan de totstandkoming van mijn proefschrift. Een aantal mensen wil ik in het bijzonder noemen.

Allereerst mijn maar liefst 4 (co-)promotoren. Het was een voorrecht op jullie expertise en ervaring te mogen terugvallen. Stef, bedankt voor je geweldige begeleiding! Ik geloof niet dat we ook maar 1 studie uitgevoerd hebben die oorspronkelijk op de planning stond, en dat geeft precies aan hoe ontzettend veel vrijheid ik gekregen heb in het vinden van mijn weg door mijn promotietraject. Bedankt voor je vertrouwen in mij, en dat je me in mijn ontwikkeling als wetenschapper gestimuleerd hebt! Ik hoop dat we nog lang kunnen blijven samenwerken. Nanne, Carel en Annette, bedankt voor jullie begeleiding, tips en adviezen, soms wat meer op afstand, maar zeker niet minder belangrijk!

Collega's bij gezondheidsbevordering: bedankt voor alle gezelligheid en adviezen. Een paar collega's verdienen toch wel een 'eigen' dankwoordje. Kathelijne: zonder jou zou het Deb niet hetzelfde zijn. Toen je vertelde dat je bij me weg ging (van onze kamer...), moest ik zowaar een traantje wegpinken. Wat is het fijn om jou als collega, vriendin en paranimf te hebben! Lieke, je bent een waardige opvolger van Kathelijne als kamergenoot. En het mooie is dat ik je zelf die formulering heb laten bedenken, zoals ik je altijd met duizend en één dingen mag lastigvallen op de meest ongebruikelijke momenten. Bovendien ben jij mijn wandelende caloriemeter, respect! 'Andere' Jessie, je bent een geweldig optimistisch mens en een fijne vriendin. Wat heerlijk dat je ook bij gezondheidsbevording bent komen werken, en natuurlijk dat je mijn paranimf bent! Tot verontwaardiging van sommige deelnemers is de colabreak nog niet eerder vermeld in het dankwoord van proefschriften van andere deelnemers. Daarom bij deze. Bilbo, Chris, Kathelijne en Rik (in alfabetische volgorde, dus nee jullie kunnen hier niet uit afleiden wie het favorietst, liefst of compatibelst is) en iedereen anders die op enig moment in de wisselende samenstelling van de colabreak heeft deelgenomen: Bedankt voor de liters cola en gespreksstof light! Ik zal nooit vergeten hoe ik bijna gestikt ben in zeven Ferrero Rochers. Ester en Sanne, bedankt voor de gezelligheid op het Deb, tijdens congressen, overleggen, vergaderingen en andere gelegenheden, en niet te vergeten alle inhoudelijke kennis die we delen! Bilbo, bedankt dat je op zo'n korte termijn zo'n supercoole kaft voor me hebt gemaakt! Leon, bedankt voor alle computeradvies, flauwekul en de laatste roddels. Marja, bedankt voor alle praktische hulp, waardoor het toch nog gelukt is mijn promotie nog voor mijn zwangerschapsverlof rond te krijgen. Ine, Renske en al jullie voorgangers, bedankt 
voor jullie ondersteuning van het KOALA cohort. Ik denk niet dat veel mensen beseffen hoe ontzettend belangrijk jullie werk is voor onderzoek.

Natuurlijk wil ik ook alle KOALA kinderen en hun ouders bedanken voor hun deelname, al die jaren en nog steeds. Ook iedereen die betrokken was bij de totstandkoming en follow-up van het KOALA cohort: Van harte bedankt! Daarnaast wil ik ook de kinderen en medewerkers van Maatwerk in Kinderopvang (MIK) bedanken voor hun medewerking en gastvrijheid tijdens de observatiestudies.

Pap en mam, bedankt dat jullie ons altijd met liefde omringd hebben en me gestimuleerd hebben het beste uit mezelf te halen. Het heeft geleid tot waar ik nu sta, en ik weet dat jullie trots op me zijn! Zussen, broer, schoonzussen, schoonbroers, schoonmam, oma, neefjes, nichtjes en andere leden van de families Gubbels en Simons: wat is het toch fijn om tot onze belachelijk grote, gezellige families te behoren! Het leven zou maar saai en eenzaam zijn zonder jullie! Vriendjes en vriendinnetjes: bedankt voor alle gezelligheid. Soms wat minder frequent door volgelopen schema's (zo gaat dat als promoverende, studerende, stagelopende, solliciterende, werkende, samenwonende, verhuizende, trouwende en/of anderszins zeer drukke 'jongvolwassenen'), maar zeker niet minder gewaardeerd!

Lieve Jef, in dit hele proefschrift sta ik vernoemd als Jessica Gubbels, maar eigenlijk ben ik natuurlijk al lang Jessica Simons. Zonder jouw onvoorwaardelijke steun en liefde had ik dit niet gekund. Wat ben ik gelukkig met jou, en wat heb ik zin in de rest van ons leven! En dan die kleine Simons in mijn buik. Laten we hopen dat je je vaders beweeggewoonten en je moeders eetgewoonten erft. Elke andere combinatie zou rampzalig zijn voor je BMI-ontwikkeling. Maar dat moeten we dan maar bijsturen met goede ouderschapspraktijken! Je hebt me in ieder geval nu al een heel andere kijk gegeven op de inhoud van mijn proefschrift. Tot snel! 


\section{Curriculum Vitae}

Jessica Sophia Gubbels was born on August 6th 1985 in Wageningen, and grew up in Margraten in the South of Limburg. After having graduated high school in 2003, she started the Bachelor Health Sciences at Maastricht University, with a major in Health Eduation and Promotion, and a minor in Movement Sciences. During her study, she participated in the Honours Programme of Health Sciences, and she graduated Cum Laude in 2006. After her graduation, Jessica started the Master Public Health at Maastricht University, with a specialisation in Health Education and Promotion. She graduated Cum Laude in 2007. On August $13^{\text {th }} 2007$, Jessica started as a PhD student at the Department of Health Promotion at Maastricht University, where she completed her dissertation in three years. 



\section{Publication list}

Van Cauwenberghe, E., Labarque, V., Gubbels, J., de Bourdeaudhuij, I. \& Cardon, G. (Submitted). Activity levels and contributing factors during preschool Physical Education.

Gubbels, J., Kremers, S., Dagnelie, P., de Vries, N., Thijs, C., Stafleu, A. \& Goldbohm, A. (2009). Invloeden van restrictieve voedingsregels van ouders op de consumptie van 2-jarige kinderen. Voeding $N u, 11(6), 10-13$.

Gubbels, J.S., Kremers, S.P.J., van Kann, D.H.H., Stafleu, A., Candel, M.J., Dagnelie, P.C., Thijs, C. \& de Vries, N.K. (In Press). Interaction between physical environment, social environment and child characteristics in determining physical activity at child-care. Health Psychology.

Gubbels, J.S., Kremers, S.P.J., Stafleu, A., Dagnelie, P.C., Goldbohm, R.A., de Vries, N.K. \& Thijs, C. (2009). Restrictive parenting practices regarding dietary intake in 2-year-old children. Impact and interactions with child characteristics. Appetite, 52, 423-429.

Gubbels, J.S., Kremers, S.P.J., Stafleu, A., Dagnelie, P.C., de Vries, N.K, van Buuren, S. \& Thijs, C. (2010). Child-care use and the association with body mass index and overweight in children 7 months to 2 years of age. International Journal of Obesity, Epub May 25th 2010.

Gubbels, J.S., Kremers, S.P.J., Stafleu, A., Dagnelie, P.C., de Vries, N.K. \& Thijs, C. (2010). Child-care environment and dietary intake of 2- and 3-year-old children. Journal of Human Nutrition and Dietetics, 23(1), 97-101 .

Gubbels, J.S., Kremers, S.P.J., Stafleu, A., Dagnelie, P.C., de Vries, N.K. \& Thijs, C. (2010). Letter to the editor. Journal of Human Nutrition and Dietetics, 23(4), 427-428.

Gubbels, J.S., Kremers, S.P.J., Stafleu, A., Dagnelie, P.C., de Vries, S.I., de Vries, N.K. \& Thijs, C. (2009). Dietary intake and sedentary behaviour already cluster in 2-year-old children. Journal of Pediatrics, 155(2), 194-198.

Gubbels, J.S., Kremers, S.P.J, Stafleu, A., de Vries, S.I., Goldbohm, R.A., Dagnelie, P.C., de Vries, N.K., van Buuren, S. \& Thijs, C. (Submitted). Impact of parenting practices on children's dietary intake, physical activity and development of body mass index: the KOALA Birth Cohort Study. 
Gubbels, J.S., Thijs, C., Stafleu, A., van Buuren, S. \& Kremers, S.P.J. (In Press). Association of breast-feeding duration and feeding on demand with child weight status up to 4 years. International Journal of Pediatric Obesity.

Stafleu, A., Goldbohm, S., van Dommelen, P., van den Bosch, L., Dutman, E., Gubbels, J., Sleddens, E. \& Thijs, C. (2009). Regels en opvoedpraktijken van ouders ten aanzien van eten - het KOALA geboortecohort. Voeding $\mathrm{Nu}, 11(10)$, 1821. 